\title{
Heterogeneous Thin Films of Martensitic Materials
}

\author{
Y. C. SHU \\ Communicated by S. MüLLER
}

\begin{abstract}
We study the effective behavior of heterogeneous thin films with three competing length scales: the film thickness and the length scales of heterogeneity and material microstructure. We start with three-dimensional nonhomogeneous nonlinear elasticity enhanced with an interfacial energy of the van der Waals type, and derive the effective energy density as all length scales tend to zero with given limiting ratios. We do not require any a priori selection of asymptotic expansion or ansatz in deriving our results. Depending on the dominating length scale, the effective energy density can be identified by three procedures: averaging, homogenization and thin-film limit. We apply our theory to martensitic materials with multi-well energy density and use a model example to show that the "shape-memory behavior" can crucially depend on the ratios of these length scales. We comment on the effective conductivity of linear composites, and also on multilayers made of shape-memory and elastic materials.
\end{abstract}

\section{Introduction}

Martensitic thin films have recently attracted much interest because of their potential for application as microactuators [27, 28,39,33, 18, 17]. Martensitic materials undergo a diffusionless phase transformation during which there is a sudden change in the crystal structure at a certain temperature. The high temperature austenite phase is cubic while the low temperature martensite phase has less symmetry. This gives rise to symmetry-related variants and these variants usually form microstructures or fine-scale mixtures. Crystals undergoing a thermoelastic martensitic transformation often exhibit the shape-memory effect. Below the transformation temperature, they are extremely malleable - sustaining a huge deformation with strains as large as $10 \%$ under very small forces. When they are heated above the transformation temperature, the specimen springs back to its original shape as 


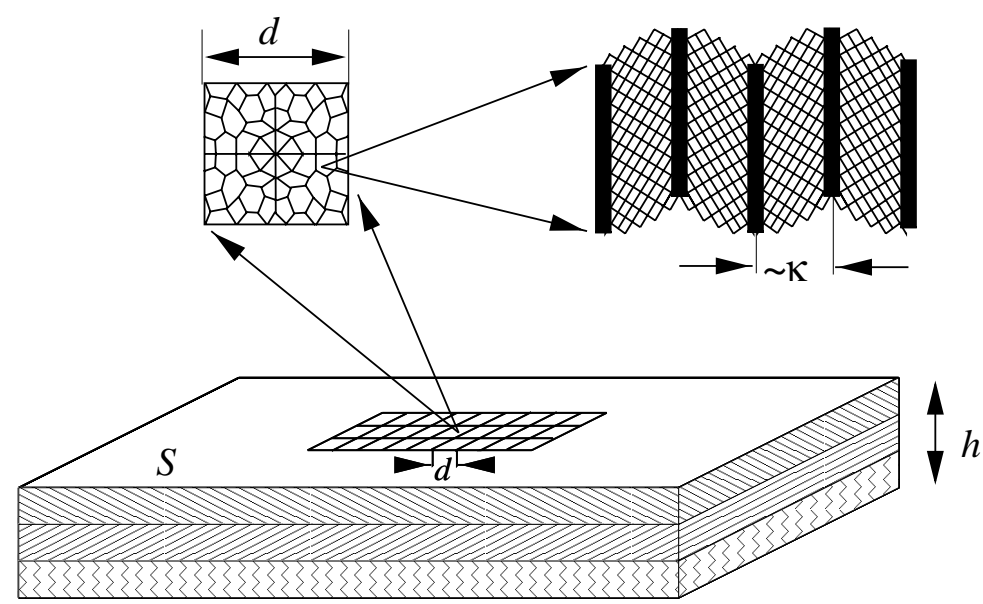

Fig. 1. A heterogeneous thin film with three different length scales.

all the strain is recovered. Actuators utilizing the shape-memory effect are predicted to have the largest energy output per unit volume per cycle among a variety of common actuator systems [33]. But bulk shape-memory actuators have enjoyed limited success in temperature sensitive applications because the response is slow due to thermal inertia. On the other hand, the enhanced rate of heat transfer in thin films makes these alloys ideal for microactuators, micropumps and microelectromechanical system (MEMS) applications.

Typically, martensitic films are polycrystalline rather than monocrystalline. A polycrystal consists of a large number of single crystal grains with different orientations. The behavior of a polycrystal can be very different from that of a single crystal because of the constraining effect of neighboring grains. Depending on the deposition technique, the size of grains within the film can be larger than, comparable to or smaller than the thickness of film. Furthermore, depending on the material, the length scale of the microstructure can also be larger than, comparable to or smaller than that of grains. The behavior of the film can critically depend on the relative magnitudes of these length scales, and we seek to understand this.

Consider a heterogeneous (possibly multilayer) thin film shown in Fig. 1. It occupies a reference domain

$$
\Omega^{h}=\left\{\mathbf{x} \in \mathbb{R}^{3}:\left(x_{1}, x_{2}\right) \in S, 0<x_{3}<h\right\},
$$

where $S$ is a bounded Lipschitz domain, $\left\{x_{1}, x_{2}, x_{3}\right\}$ are relative to an orthonormal film basis $\left\{\mathbf{e}_{\mathbf{1}}, \mathbf{e}_{\mathbf{2}}, \mathbf{e}_{\mathbf{3}}\right\}$, and $h$ is the film thickness. Let $\tilde{\mathbf{y}}: \Omega^{h} \rightarrow \mathbb{R}^{3}$ be the deformation of the film. The total energy of the heterogeneous thin film is

$$
\tilde{e}^{(h)}[\tilde{\mathbf{y}}]=\int_{\Omega^{h}}\left\{\kappa^{2}\left|\nabla^{2} \tilde{\mathbf{y}}\right|^{2}+\varphi\left(\nabla \tilde{\mathbf{y}}, \frac{x_{1}}{d}, \frac{x_{2}}{d}, \frac{x_{3}}{h}\right)\right\} d \mathbf{x}
$$

where $\varphi: \mathbb{M}^{3 \times 3} \times \mathbb{R}^{2} \times(0,1) \rightarrow \mathbb{R}$ is the elastic free energy density of the film and $\mathbb{M}^{m \times n}$ is the set of all $m \times n$ matrices. We assume that $\varphi$ is periodic in the 
in-plane variables $x_{1}$ and $x_{2}$ with period $[0,1]^{2}$. So $d$ scales like the typical grain size. Further, since we wish to model martensites, $\varphi(\mathbf{F}, \cdot, \cdot, \cdot)$ may have a multi-well structure and consequently nonconvex energy densities. Note that we have included the interfacial energy of the type $\kappa^{2}\left|\nabla^{2} \tilde{\mathbf{y}}\right|^{2}$. Minimizers of the energy (1.2) have oscillations on a length scale that scales with $\kappa$ and hence we call $\kappa$ the length scale of the microstructure. We are interested in finding the limiting behavior of the film when all length scales $\kappa, d$ and $h$ tend to zero. Therefore, we take

$$
\kappa=\kappa(h)>0, \quad d=d(h)>0, \quad \lim _{h \rightarrow 0} \kappa(h)=0, \quad \lim _{h \rightarrow 0} d(h)=0,
$$

and assume that they have fixed limiting ratios:

$$
\alpha=\lim _{h \rightarrow 0} \frac{\kappa}{d}, \quad \beta=\lim _{h \rightarrow 0} \frac{h}{d}, \quad \alpha^{\prime}=\lim _{h \rightarrow 0} \frac{\kappa}{h} .
$$

In bulk materials, the homogenization of cellular elastic materials with nonconvex energy density $\varphi$ has been studied by BraIDEs [13] and Müller [36]. The same problem including the interfacial energy has been studied by FRANCFORT \& MüLLER [24]. However, microstructure in thin films can be significantly different to that in bulk materials, endowing materials with dramatically distinct properties (for example, see [3]). Recently, BhatTACHARYA \& JAMEs [9] have developed a theory of single crystal martensitic thin films which captures this effect. Related work on the modeling of thin structures with convex (quadratic) energy density includes, for example, Kohn \& Vogelius [31,32], Damlamian \& Vogelius [20] and CAILLERIE [16]; and related problems with nonconvex energy density include, for example, Acerbi et al. [1], Le Dret \& Raoult [21] and Fonseca \& FrancFORT [22]. We wish to combine homogenization with the thin-film analysis for nonconvex energies and apply it to heterogeneous martensitic films. BRAIDES, FONSECA $\&$ FRANCFORT [15] have studied a similar problem with $\kappa=0$.

Our approach is variational. We study the "variational limit" of (1.2) as $h$ tends to zero. Since the energy defined in (1.2) scales like $h$ as $h$ tends to zero, we shall be interested in the limiting energy per unit thickness, i.e.,

$$
\tilde{e}_{1}^{(h)}=\frac{1}{h} \tilde{e}^{(h)} .
$$

We expect the minimum values and the minimizers of the functional $\tilde{e}_{1}^{(h)}$ to converge to those of a "limiting energy" $\tilde{e}_{1}^{(0)}$, which we try to find. In this context, the natural tool is $\Gamma$-convergence as proposed by De Giorgi [25] and De Giorgi \& FRANZONI [26] which under a suitable technical hypothesis is nearly identical to that of convergence of minimizers (see also Remark 1). Using this notion, we show that the limiting energy is always given by

$$
\tilde{e}_{1}^{(0)}[\mathbf{y}]=\int_{S} \bar{\varphi}\left(\frac{\partial \mathbf{y}}{\partial x_{1}}, \frac{\partial \mathbf{y}}{\partial x_{2}}\right) d x_{1} d x_{2},
$$

where $\bar{\varphi}$ is the effective energy density and only depends on the in-plane gradient of deformation $\mathbf{y}$ and not explicitly on the position. It describes the overall behavior of 
Table 1. Summary of the effective behavior of a heterogeneous thin film. A means averaging, $\mathbf{H}$ means homogenizing and $\mathbf{T}$ means thin-film limit. TH denotes that the effective energy density $\bar{\varphi}$ is obtained by taking the thin-film limit first, and then homogenizing in the plane of the film. On the other hand, HT denotes homogenization first followed by the thin-film limit. Finally, a stacked symbol ${ }_{T}^{H}$ denotes the simultaneous performance of these two operations.

\begin{tabular}{|c|c|c|c|}
\hline$\kappa \gg d$ & $\begin{array}{l}\text { Theorem } 1 \\
\qquad \mathbf{A ~ T}\end{array}$ & $\begin{array}{l}\text { Theorem } 1 \\
\qquad \mathbf{A ~ T}\end{array}$ & 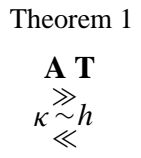 \\
\hline$\kappa \sim d$ & $\begin{array}{c}\text { Theorem } 2 \\
{ }_{\mathbf{T}}^{\mathbf{A}} \\
\mathbf{H}\end{array}$ & $\begin{array}{c}\text { Theorem } 3 \\
\mathbf{A} \\
\mathbf{H} \\
\mathbf{T}\end{array}$ & $\underbrace{\mathbf{A}_{\text {( ) }}}_{(?)} \mathbf{T}$ \\
\hline$\kappa \ll d$ & $\begin{array}{c}\text { Theorem } 2 \\
\mathbf{T} \mathbf{H} \\
\gg h \\
\ll \\
\ll \\
\text { Theorem } 4\end{array}$ & $\begin{array}{c}\text { Theorem } 3 \\
\mathbf{H} \\
\mathbf{T}\end{array}$ & $\begin{array}{c}\text { Theorem } 5 \\
\text { H } \mathbf{T}\end{array}$ \\
\hline & $h \ll d$ & $h \sim d$ & $h \gg d$ \\
\hline
\end{tabular}

the heterogeneous thin film after taking into account the martensitic microstructure, grains and multilayers.

In the following, we give a non-technical description of our main results which are summarized in Table 1. The most important finding is that the effective energy density $\bar{\varphi}$ crucially depends on the limiting ratios of these three length scales.

1. Strong interfacial energy $(\kappa>>d)$. Assume $\varphi=\varphi\left(\mathbf{F}, \frac{x_{1}}{d}, \frac{x_{2}}{d}\right)$. Our Theorem 1 shows that the effective energy density $\bar{\varphi}$ is obtained by averaging the microscopic energy $\varphi$ over the period, then passing to the thin-film limit. It costs materials more energy to form microstructures within each grain as a result of strong interfacial energy. Material is internally stressed. The result is also true if $\varphi=\varphi\left(\mathbf{F}, \frac{x_{1}}{d}, \frac{x_{2}}{d}, \frac{x_{3}}{h}\right)$ and if $\kappa>>d$ and $\kappa>>h$.

2. Flat grains $(d>>h)$. Assume $\varphi=\varphi\left(\mathbf{F}, \frac{x_{1}}{d}, \frac{x_{2}}{d}\right)$. If the length scale of the microstructure is much smaller than that of grains (i.e., if $\kappa<<d$ ), then Theorem 2(i) shows that the elastic energy dominates the interfacial energy and materials can form microstructures freely. As a result, the macroscopic energy density $\bar{\varphi}$ is impervious to the presence of interfacial energy. Further, $\bar{\varphi}$ is obtained by taking the thin-film limit first, and then homogenizing in the plane of the film. The thin-film limit says that only the in-plane compatibility is important and this allows a wider class of microstructures to be formed in thin films than in bulk materials. On the other hand, if the length scales of grains and microstructure are of the same order of magnitude (i.e., if $\kappa \approx d$ ), Theorem 2(ii) shows that the interfacial energy explicitly contributes to the effective energy 
density $\bar{\varphi}$. Materials can form only a limited amount of microstructure because of competing energies between elastic energy and interfacial energy.

3. Comparable grains $(d \approx h)$. Assume $\varphi=\varphi\left(\mathbf{F}, \frac{x_{1}}{d}, \frac{x_{2}}{d}, \frac{x_{3}}{h}\right)$. Our Theorem 3 gives the expression of effective energy density $\bar{\varphi}$ when all length scales are comparable. This case apparently has no simple explanation since the averaging, homogenizing and thin-film limit are taken into account together.

4. Long grains $(d<<h)$. Assume $\varphi=\varphi\left(\mathbf{F}, \frac{x_{1}}{d}, \frac{x_{2}}{d}\right)$. Theorem 1 includes the case $d<<\kappa$. On the other hand, if $\kappa=0$ or $\kappa<<d$, Theorem 5 says that the effective energy density is obtained by homogenizing the bulk material, then passing to the thin-film limit. Finally, if $\kappa$ and $d$ are of the same order of magnitude and both are much smaller than $h(\kappa \approx d<<h)$, we conjecture that the effective energy density is obtained by taking averaging and bulk homogenization first, and then passing to the thin-film limit.

5. Multilayers $(\kappa$ versus $h)$. Assume $\varphi=\varphi\left(\mathbf{F}, \frac{x_{3}}{h}\right)$. In such a situation, only two physical parameters $\kappa$ and $h$ are relevant. Our Theorem 4 gives the expression of $\bar{\varphi}$ containing through-the-thickness variations.

We apply our results in Section 7. We use examples to show that the macroscopic behavior of films can significantly depend on the limiting ratios of these length scales. In our first example, we are interested in the shape-memory behavior of a polycrystalline martensitic film. Shape-memory materials are modeled with a multiwell energy density $\varphi$, each well representing a phase or variant. The relaxation of $\varphi$ has the degeneracy, i.e., $Q \varphi=0$ on a set $\mathcal{S}$. This set $\mathcal{S}$ contains all strains recoverable on heating in a single crystal. Similarly, the strains recoverable on heating in a polycrystal are contained in the set $\mathcal{P}$ on which $\bar{\varphi}$ vanishes [11]. While the set $\mathcal{S}$ can be obtained in most martensitic materials, the set $\mathcal{P}$ is rather difficult to calculate. The estimation of this set $\mathcal{P}$ in bulk martensitic materials have been studied in $[10,11,37]$. We extend this framework to thin films. Our result shows that, for strong interfacial energy (i.e., $\kappa>>d$ ), the shape-memory behavior is expected to be negligible in general polycrystals since materials cannot form microstructures within each grain to accommodate deformation. On the other hand, for small interfacial energy (i.e., $\kappa<<d$ ), materials can form microstructures freely and our model example shows that this set $\mathcal{P}$ significantly depends on the limiting ratio of $\frac{h}{d}$. We further consider cubic-monoclinic shape-memory thin films. We show that recoverable strains in thin films with flat columnar grains $(d>>h)$ differs from (are larger than) those with long columnar grains $(d<<h)$. We also establish that films made by sputtering can recover only relatively small strains in Ti-Ni and other common shape-memory alloys.

Next, we consider effective conductivity of linear composites. We show that in general the effective conductivity of composites made of anisotropic materials can depend on the ratio $\frac{h}{d}$. We also provide bounds to estimate it in our model example. We compare this result with the optimal bounds of DamLAmian \& Vogelius [20].

Finally, we consider a multilayered thin film made of a finite number of alternating layers of a martensitic material and a purely elastic material. We find quite different behavior when $\frac{\kappa}{h}$ tends to zero and infinity. We conclude that such 
a multilayered thin film provides an opportunity to design materials with unusual transformation properties.

\section{Preliminaries}

It is convenient to work on a fixed domain instead of a varying domain $\Omega^{h}$, so we introduce the following change of variables:

$$
\mathbf{z}_{p}=\left(z_{1}, z_{2}\right)=\mathbf{x}_{p}=\left(x_{1}, x_{2}\right), \quad z_{3}=\frac{1}{h} x_{3}, \quad \mathbf{x} \in \Omega^{h},
$$

and set

$$
\Omega^{1}=S \times(0,1) .
$$

With each deformation $\tilde{\mathbf{y}}: \Omega^{h} \rightarrow \mathbb{R}^{3}$ we associate a deformation $\mathbf{y}: \Omega^{1} \rightarrow \mathbb{R}^{3}$ via

$$
\mathbf{y}(\mathbf{z}(\mathbf{x}))=\tilde{\mathbf{y}}(\mathbf{x}), \quad \mathbf{x} \in \Omega^{h} .
$$

We use the notation $\nabla_{p}$ for the gradient in the plane of the film, i.e.,

$$
\nabla_{p} \mathbf{y}=\mathbf{y}_{, 1} \otimes \mathbf{e}_{1}+\mathbf{y}_{, 2} \otimes \mathbf{e}_{2},
$$

and $\mathbf{y}_{, 1}=\frac{\partial \mathbf{y}}{\partial z_{1}}=\left(\frac{\partial y_{1}}{\partial z_{1}}, \frac{\partial y_{2}}{\partial z_{1}}, \frac{\partial y_{3}}{\partial z_{1}}\right)^{T}$; etc. We now change variables in $\frac{1}{h} \tilde{e}^{(h)}$ using (1.2) and (2.1) and get

$$
\begin{aligned}
\tilde{e}_{1}^{(h)}[\mathbf{y}]:= & \frac{1}{h} \tilde{e}^{(h)}[\tilde{\mathbf{y}}] \\
= & \int_{\Omega^{1}}\left\{\kappa^{2}\left(\left|\nabla_{p}^{2} \mathbf{y}\right|^{2}+\frac{2}{h^{2}}\left|\nabla_{p} \mathbf{y}_{, 3}\right|^{2}+\frac{1}{h^{4}}\left|\mathbf{y}_{, 33}\right|^{2}\right)\right. \\
& \left.+\varphi\left(\mathbf{y}_{, 1}|\mathbf{y}, 2| \frac{1}{h} \mathbf{y}_{, 3}, \frac{\mathbf{z}_{p}}{d}, z_{3}\right)\right\} d \mathbf{z} .
\end{aligned}
$$

We have used the notation

$$
\mathbf{F}=\left(\mathbf{f}_{1}\left|\mathbf{f}_{2}\right| \mathbf{f}_{3}\right)=\mathbf{f}_{1} \otimes \mathbf{e}_{1}+\mathbf{f}_{2} \otimes \mathbf{e}_{2}+\mathbf{f}_{3} \otimes \mathbf{e}_{3}
$$

for $\mathbf{F} \in \mathbb{M}^{3 \times 3}$.

We assume the energy density $\varphi$ satisfies the following conditions:

1. $\varphi(\mathbf{F}, \mathbf{z})$ is Carathéodory and nonnegative.

2. Periodicity in the plane of the film: $\varphi\left(\mathbf{F}, \mathbf{z}_{p}, z_{3}\right)$ is periodic in the in-plane variable $\mathbf{z}_{p}=\left(z_{1}, z_{2}\right)$ with period $[0,1]^{2}$ for all $\mathbf{F} \in \mathbb{M}^{3 \times 3}$ and $z_{3} \in(0,1)$.

3. Growth and coercivity conditions:

$$
c_{1}\left(|\mathbf{F}|^{p}-1\right) \leqq \varphi(\mathbf{F}, \mathbf{z}) \leqq c_{2}\left(|\mathbf{F}|^{p}+1\right)
$$

for all $\mathbf{F} \in \mathbb{M}^{3 \times 3}$ and for a.e. $\mathbf{z}=\left(\mathbf{z}_{p}, z_{3}\right) \in \mathbb{R}^{2} \times(0,1)$. 
4. Lipschitz condition:

$$
|\varphi(\mathbf{F}, \mathbf{z})-\varphi(\mathbf{G}, \mathbf{z})| \leqq c_{2}\left(1+|\mathbf{F}|^{p-1}+|\mathbf{G}|^{p-1}\right)|\mathbf{F}-\mathbf{G}|
$$

for all $(\mathbf{F}, \mathbf{G}) \in \mathbb{M}^{3 \times 3} \times \mathbb{M}^{3 \times 3}$ and for a.e. $\mathbf{z}=\left(\mathbf{z}_{p}, z_{3}\right) \in \mathbb{R}^{2} \times(0,1)$.

Above, $0<c_{1} \leqq c_{2}$ and $p$ satisfies $1<p<\infty$.

For any $\mathbf{y} \in W^{1, p}\left(\Omega^{1}, \mathbb{R}^{3}\right)$, we extend the functional $\tilde{e}_{1}^{(h)}[\mathbf{y}]$ to

$$
e_{1}^{(h)}[\mathbf{y}]= \begin{cases}\tilde{e}_{1}^{(h)}[\mathbf{y}] & \text { if } \mathbf{y} \in W^{2,2}\left(\Omega^{1}, \mathbb{R}^{3}\right) \\ +\infty & \text { otherwise }\end{cases}
$$

Now our goal is to compute the $\Gamma$-limit of $e_{1}^{(h)}$ as $h, d$ and $\kappa$ tend to zero with fixed limiting ratios (1.4). To this purpose, we recall that

Definition 1. A family $e_{1}^{(h)}$ of functionals on $W^{1, p}\left(\Omega^{1}, \mathbb{R}^{3}\right)(1<p<\infty)$ is said to $\Gamma$-converge (in the weak $W^{1, p}\left(\Omega^{1}, \mathbb{R}^{3}\right)$ topology) to $e_{1}^{(0)}$ if and only if

(I) every sequence $\mathbf{y}^{(h)}$ with

$$
\mathbf{y}^{(h)} \rightarrow \mathbf{y} \quad \text { in } \quad W^{1, p}\left(\Omega^{1}, \mathbb{R}^{3}\right) \quad \text { as } h \rightarrow 0,
$$

satisfies the "lower bound"

$$
\liminf _{h \rightarrow 0} e_{1}^{(h)}\left[\mathbf{y}^{(h)}\right] \geqq e_{1}^{(0)}[\mathbf{y}]
$$

(II) for every $\mathbf{y} \in W^{1, p}\left(\Omega^{1}, \mathbb{R}^{3}\right)$, there exists a sequence $\mathbf{y}^{(h)}$ called the "recovery sequence" such that

$$
\mathbf{y}^{(h)} \rightarrow \mathbf{y} \quad \text { in } \quad W^{1, p}\left(\Omega^{1}, \mathbb{R}^{3}\right) \quad \text { as } h \rightarrow 0
$$

and

$$
\lim _{h \rightarrow 0} e_{1}^{(h)}\left[\mathbf{y}^{(h)}\right]=e_{1}^{(0)}[\mathbf{y}]
$$

Remark 1. The limiting functional $e_{1}^{(0)}$ is, by construction, lower semicontinuous with respect to weak convergence in $W^{1, p}\left(\Omega^{1}, \mathbb{R}^{3}\right)$ [14] and, therefore, attains its minimum value due to the coercivity condition (2.4). Further, using the fact that the $L^{2}$ norm is sequentially lower semicontinuous and Rellich's compactness theorem, one can show that $e_{1}^{(h)}$ admits a minimum for any fixed $h>0$ (cf. FRANCFORT \& MÜLLER [24]). Therefore, minimizers of $e_{1}^{(h)}$ converge to those of $e_{1}^{(0)}$ by the fundamental theorem of $\Gamma$-convergence (see, for example, BRAIDES \& DEFRANCESCHI [14]). 
In the following, we will show that, for any $\mathbf{y} \in W^{1, p}\left(\Omega^{1}, \mathbb{R}^{3}\right), e_{1}^{(h)} \Gamma$-converges to a functional $e_{1}^{(0)}$ of the form

$$
e_{1}^{(0)}[\mathbf{y}]= \begin{cases}\int_{S} \bar{\varphi}\left(\nabla_{p} \mathbf{y}\right) d \mathbf{z}_{p} & \text { if } \mathbf{y} \in V_{S}, \\ +\infty & \text { otherwise }\end{cases}
$$

where $\bar{\varphi}$ will be determined explicitly and $V_{S}$ is defined by

$$
V_{S}=\left\{\mathbf{y}: \mathbf{y} \in W^{1, p}\left(\Omega^{1}, \mathbb{R}^{3}\right) \quad \text { and } \mathbf{y}_{, 3}=0 \text { for a.e. } \mathbf{z} \text { in } \Omega^{1}\right\}
$$

which is canonically isomorphic to $W^{1, p}\left(S, \mathbb{R}^{3}\right)$. The following lemma is the first step towards proving (2.7); with it we only need to compute the $\Gamma$-limit of $e_{1}^{(h)}$ for $\mathbf{y} \in V_{S}$.

Lemma 1. Let $e_{1}^{(h)}$ be defined by (2.6) and assume $\mathbf{y} \notin V_{S}$. Then, $\liminf _{h \rightarrow 0} e_{1}^{(h)}$ $\left[\mathbf{y}^{(h)}\right]=+\infty$ for any sequence $\mathbf{y}^{(h)}$ such that $\mathbf{y}^{(h)} \rightarrow \mathbf{y}$ in $W^{1, p}\left(\Omega^{1}, \mathbb{R}^{3}\right)$ as $h \rightarrow 0$.

Proof. We prove it by contradiction. Suppose there exists a sequence $\mathbf{y}^{(h)}$ converging weakly to $\mathbf{y}$ in $W^{1, p}\left(\Omega^{1}, \mathbb{R}^{3}\right)$ with $\liminf _{h \rightarrow 0} e_{1}^{(h)}\left[\mathbf{y}^{(h)}\right]=M$ finite. Therefore, there exists a subsequence $\mathbf{y}^{(h)}$ (not relabeled) such that

$$
e_{1}^{(h)}\left[\mathbf{y}^{(h)}\right] \rightarrow M<+\infty \quad \text { as } h \rightarrow 0 .
$$

By coercivity, $\frac{1}{h} \mathbf{y}_{, 3}^{(h)}$ is bounded in $L^{p}\left(\Omega^{1}, \mathbb{R}^{3}\right)$ and this implies

$$
\mathbf{y}_{, 3}^{(h)} \rightarrow 0 \quad \text { strongly in } L^{p}\left(\Omega^{1}, \mathbb{R}^{3}\right)
$$

as $h \rightarrow 0$. Since $\mathbf{y}^{(h)}$ converges weakly to $\mathbf{y}$ in $W^{1, p}\left(\Omega^{1}, \mathbb{R}^{3}\right)$ as $h$ tends to zero, this gives

$$
\mathbf{y}_{, 3}^{(h)} \stackrel{h}{\rightarrow} \mathbf{y}_{, 3} \quad \text { in } L^{p}\left(\Omega^{1}, \mathbb{R}^{3}\right) .
$$

Combining (2.9) and (2.10), we have $\mathbf{y}_{, 3}=0$ a.e. by the uniqueness of the weak limit. Thus $\mathbf{y} \in V_{S}$, which contradicts the assumption, and this completes the proof.

\section{Strong interfacial energy}

Theorem 1. Let $e_{1}^{(h)}$ and $e_{1}^{(0)}$ be defined by (2.6) and (2.7). Then, $e_{1}^{(h)} \Gamma$-converges to the functional $e_{1}^{(0)}$ if

(i) $\varphi=\varphi\left(\mathbf{F}, \frac{\mathbf{z}_{p}}{d}\right), \frac{\kappa}{d} \rightarrow \infty$ as $h \rightarrow 0$, and

$$
\begin{aligned}
\bar{\varphi}(\overline{\mathbf{F}}) & =Q \tilde{\varphi}_{0}(\overline{\mathbf{F}}), \\
\tilde{\varphi}_{0}(\overline{\mathbf{F}}) & =\inf _{\mathbf{b} \in \mathbb{R}^{3}} \tilde{\varphi}(\overline{\mathbf{F}} \mid \mathbf{b}), \\
\tilde{\varphi}(\mathbf{F}) & =\int_{Z} \varphi\left(\mathbf{F}, \mathbf{z}_{p}\right) d \mathbf{z}_{p},
\end{aligned}
$$

where $Q \tilde{\varphi}_{0}$ is the lower quasi-convex envelope of $\tilde{\varphi}_{0}, \overline{\mathbf{F}} \in \mathbb{M}^{3 \times 2}$ and $Z=$ $(0,1)^{2}$; 
(ii) $\varphi=\varphi\left(\mathbf{F}, \frac{\mathbf{z}_{p}}{d}, z_{3}\right), \frac{\kappa}{d} \rightarrow \infty, \frac{\kappa}{h} \rightarrow \infty$ as $h \rightarrow 0$, and $\bar{\varphi}$ is given by $(3.1)_{1}$, $(3.1)_{2}$ with $(3.1)_{3}$ replaced by

$$
\tilde{\varphi}(\mathbf{F})=\int_{Z \times(0,1)} \varphi(\mathbf{F}, \mathbf{z}) d \mathbf{z}
$$

(iii) $\varphi=\varphi\left(\mathbf{F}, z_{3}\right), \frac{\kappa}{h} \rightarrow \infty$ as $h \rightarrow 0$, and $\bar{\varphi}$ is given by $(3.1)_{1},(3.1)_{2}$ with $(3.1)_{3}$ replaced by

$$
\tilde{\varphi}(\mathbf{F})=\int_{0}^{1} \varphi\left(\mathbf{F}, z_{3}\right) d z_{3} .
$$

Remark 2. It is clear that $\tilde{\varphi}(\mathbf{F})$ enjoys the same growth and coercivity conditions (2.4) and is continuous by virtue of the Lipschitz condition (2.5) on $\varphi$. It follows that $\tilde{\varphi}_{0}$ given by $(3.1)_{2}$ is well defined and the infimum is achieved. Further, Proposition 1 of LE DRET \& RAOUlt [21] shows that $\tilde{\varphi}_{0}(\overline{\mathbf{F}})$ satisfies the growth and coercivity estimates (2.4) and is continuous.

Proof of Theorem 1. We begin with case (i): $\varphi=\varphi\left(\mathbf{F}, \frac{\mathbf{z}_{p}}{d}\right)$ and $\frac{\kappa}{d} \rightarrow \infty$ as $h \rightarrow 0$. We first construct a recovery sequence for any $\mathbf{y} \in V_{S}$. Recalling Remark 2 and invoking the relaxation theorem due to DACOROGNA [19] we find a sequence $\mathbf{y}^{(\delta)}$ which converges weakly to $\mathbf{y}$ in $W^{1, p}\left(S, \mathbb{R}^{3}\right)$ such that

$$
\int_{S} \tilde{\varphi}_{0}\left(\nabla_{p} \mathbf{y}^{(\delta)}\right) d \mathbf{z}_{p} \rightarrow \int_{S} Q \tilde{\varphi}_{0}\left(\nabla_{p} \mathbf{y}\right) d \mathbf{z}_{p} \quad \text { as } \delta \rightarrow 0 .
$$

Since the infimum of $\tilde{\varphi}_{0}$ is achieved (see Remark 2), an argument like that used by LE DReT \& RAOult [21] shows that for each element of the sequence $\mathbf{y}^{(\delta)}$, there exists a measurable $\mathbf{b}^{(\delta)} \in L^{p}\left(S, \mathbb{R}^{3}\right)$ such that

$$
\tilde{\varphi}_{0}\left(\nabla_{p} \mathbf{y}^{(\delta)}\right)=\tilde{\varphi}\left(\nabla_{p} \mathbf{y}^{(\delta)} \mid \mathbf{b}^{(\delta)}\right)
$$

Further, we may also assume at the moment that both $\mathbf{y}^{(\delta)}\left(\mathbf{z}_{p}\right)$ and $\mathbf{b}^{(\delta)}\left(\mathbf{z}_{p}\right)$ are smooth functions because of the Lipschitz character of $\partial S$ (see Remark 3). Define

$$
\mathbf{y}^{(\delta, h)}=\mathbf{y}^{(\delta)}\left(\mathbf{z}_{p}\right)+h \mathbf{b}^{(\delta)}\left(\mathbf{z}_{p}\right) z_{3}
$$

and substitute it into $e_{1}^{(h)}$. We have

$$
\begin{aligned}
e_{1}^{(h)}\left[\mathbf{y}^{(\delta, h)}\right]= & \int_{\Omega_{1}}\left\{\kappa^{2}\left(\left|\nabla_{p}^{2} \mathbf{y}^{(\delta)}+h \nabla_{p}^{2} \mathbf{b}^{(\delta)} z_{3}\right|^{2}+2\left|\nabla_{p} \mathbf{b}^{(\delta)}\right|^{2}\right)\right. \\
& \left.+\varphi\left(\nabla_{p} \mathbf{y}^{(\delta)}+h \nabla_{p}\left(\mathbf{b}^{(\delta)} z_{3}\right) \mid \mathbf{b}^{(\delta)}, \frac{\mathbf{z}_{p}}{d}\right)\right\} d \mathbf{z} .
\end{aligned}
$$

The first term of the integrand, $\kappa^{2}\left(\left|\nabla_{p}^{2} \mathbf{y}^{(\delta)}+h \nabla_{p}^{2} \mathbf{b}^{(\delta)} z_{3}\right|^{2}+2\left|\nabla_{p} \mathbf{b}^{(\delta)}\right|^{2}\right)$, vanishes for any fixed $\delta$ since $\kappa(h) \rightarrow 0$ as $h \rightarrow 0$. Therefore, using the Lipschitz condition 
(2.5) on $\varphi$, we get

$$
\begin{aligned}
e_{1}^{(h)}\left[\mathbf{y}^{(\delta, h)}\right] & \stackrel{h}{\longrightarrow} \int_{S} \int_{Z} \varphi\left(\nabla_{p} \mathbf{y}^{(\delta)}\left(\mathbf{z}_{p}\right) \mid \mathbf{b}^{(\delta)}\left(\mathbf{z}_{p}\right), \hat{\mathbf{z}}_{p}\right) d \hat{\mathbf{z}}_{p} d \mathbf{z}_{p} \\
& =\int_{S} \tilde{\varphi}\left(\nabla_{p} \mathbf{y}^{(\delta)}\left(\mathbf{z}_{p}\right) \mid \mathbf{b}^{(\delta)}\left(\mathbf{z}_{p}\right)\right) d \mathbf{z}_{p} \\
& =\int_{S} \tilde{\varphi}_{0}\left(\nabla_{p} \mathbf{y}^{(\delta)}\left(\mathbf{z}_{p}\right)\right) d \mathbf{z}_{p} .
\end{aligned}
$$

Above in (3.7), we have approximated $\left(\nabla_{p} \mathbf{y}^{(\delta)} \mid \mathbf{b}^{(\delta)}\right)$ by a piecewise constant element in $L^{p}\left(S, \mathbb{R}^{9}\right)$, passed to the limit as in (3.8) using the Lemma A.1 by BALL $\&$ Murat $[7]^{1}$, and then use the estimate $(2.5)$ on $\varphi$ again to complete the whole argument.

Recalling (3.4) gives us

$$
\limsup _{\delta \rightarrow 0} \limsup _{h \rightarrow 0} e_{1}^{(h)}\left[\mathbf{y}^{(\delta, h)}\right]=e_{1}^{(0)}[\mathbf{y}] .
$$

Now appealing to the standard diagonalization argument of AтTOUCH [4, Corollary $1.16]$ yields a sequence $\mathbf{y}^{(\delta(h))}$ that converges weakly to $\mathbf{y}$ in $W^{1, p}\left(\Omega^{1}, \mathbb{R}^{3}\right)$ as $h \rightarrow 0$ and satisfies

$$
\lim _{h \rightarrow 0} e_{1}^{(h)}\left[\mathbf{y}^{(\delta(h))}\right]=e_{1}^{(0)}[\mathbf{y}]
$$

To complete the proof, we need to establish the lower bound. Let $\mathbf{y}^{(h)} \stackrel{h}{\rightarrow} \mathbf{y} \in V_{S}$ in $W^{1, p}\left(\Omega^{1}, \mathbb{R}^{3}\right)$. We may assume that $\liminf _{h \rightarrow 0} e_{1}^{(h)}\left[\mathbf{y}^{(h)}\right]$ is finite; else the result follows. We may also restrict ourselves to a subsequence $\mathbf{y}^{(h)}$ (not relabeled) which achieves the lim inf.

For any $\delta>0$ let $S^{\prime} \subset \subset S$ with $\left|S \backslash S^{\prime}\right|<\delta$. Define

$$
\begin{gathered}
P^{d}=\left\{\mathbf{z}_{p} \in d \mathbb{Z}^{2}: \mathbf{z}_{p}+d Z \subset S^{\prime}\right\}, \\
S^{d}=\bigcup_{\mathbf{z}_{p} \in P^{d}}\left(\mathbf{z}_{p}+d Z\right), \\
\Omega^{1, d}=S^{d} \times(0,1) \text { and } \Omega^{\prime}=S^{\prime} \times(0,1) .
\end{gathered}
$$

Clearly $S^{d} \subset S^{\prime}$. For each $\hat{\mathbf{z}}$ in $\Omega^{1, d}$ define

$$
\mathbf{Y}^{(h)}\left(\hat{\mathbf{z}}_{p}, \hat{z}_{3}\right)=\frac{1}{d^{2}} \int_{\mathbf{z}_{p}+d Z}\left(\nabla_{p} \mathbf{y}^{(h)} \mid \frac{1}{h} \mathbf{y}_{, 3}^{(h)}\right) d \tilde{\mathbf{z}}_{p}, \quad \hat{\mathbf{z}}_{p} \in \mathbf{z}_{p}+d Z, \quad \mathbf{z}_{p} \in P^{d}
$$

One can check easily that

$$
\left\|\mathbf{Y}^{(h)}\right\|_{L^{p}\left(\Omega^{1, d}\right)} \leqq\left\|\left(\nabla_{p} \mathbf{y}^{(h)} \mid \frac{1}{h} \mathbf{y}_{, 3}^{(h)}\right)\right\|_{L^{p}\left(\Omega^{1, d}\right)} .
$$

1 Suppose $1 \leqq p \leqq \infty$. Let $g(\mathbf{x}) \in L_{\text {loc }}^{p}\left(\mathbb{R}^{m}\right)$ be $[0,1]^{m}$-periodic. Then $g\left(\frac{\mathbf{x}}{\varepsilon}\right)$ converges weakly in $L^{p}(\Omega)$ to its mean value as $\varepsilon \rightarrow 0$ for any bounded open subset $\Omega$. 
Using the Poincaré inequality for each small square in $S^{d}$ at fixed $z_{3}$, summing all such squares, and integrating over $z_{3}$ from 0 to 1 , one can deduce that

$$
\begin{aligned}
& \left\|\mathbf{Y}^{(h)}-\left(\nabla_{p} \mathbf{y}^{(h)} \mid \frac{1}{h} \mathbf{y}_{, 3}^{(h)}\right)\right\|_{L^{2}\left(\Omega^{1, d}\right)}^{2} \\
& \leqq C\left(\frac{d}{\kappa}\right)^{2} \int_{\Omega^{1, d}} \kappa^{2}\left(\left|\nabla_{p}^{2} \mathbf{y}^{(h)}\right|^{2}+\frac{1}{h^{2}}\left|\nabla_{p} \mathbf{y}_{, 3}^{(h)}\right|^{2}\right) d \mathbf{z}
\end{aligned}
$$

where $C$ is some constant that does not depend on $h$. Using the fact that $\frac{d}{\kappa} \rightarrow 0$ as $h \rightarrow 0$ and the finiteness of $\liminf _{h \rightarrow 0} e_{1}^{(h)}\left[\mathbf{y}^{(h)}\right]$, we have

$$
\left\|\mathbf{Y}^{(h)}-\left(\nabla_{p} \mathbf{y}^{(h)} \mid \frac{1}{h} \mathbf{y}_{, 3}^{(h)}\right)\right\|_{L^{2}\left(\Omega^{1, d}\right)} \rightarrow 0 \quad \text { as } h \rightarrow 0 .
$$

Thus, we can apply Egoroff's theorem to assert the existence of a measurable subset $A$ of $\Omega^{\prime}$ such that, for sufficiently small $h, A \subset \Omega^{1, d},\left|\Omega^{\prime} \backslash A\right|<\delta$ and

$$
\mathbf{Y}^{(h)}-\left(\nabla_{p} \mathbf{y}^{(h)} \mid \frac{1}{h} \mathbf{y}_{, 3}^{(h)}\right) \rightarrow 0 \quad \text { uniformly on } A
$$

as $h \rightarrow 0$ for some subsequence $\left(\mathbf{Y}^{(h)}-\left(\nabla_{p} \mathbf{y}^{(h)} \mid \frac{1}{h} \mathbf{y}_{, 3}^{(h)}\right)\right)$ (not relabeled). Using the Lipschitz condition (2.5), (3.12), (3.15) and the uniform boundedness of $\left\|\left(\nabla_{p} \mathbf{y}^{(h)} \mid \frac{1}{h} \mathbf{y}_{, 3}^{(h)}\right)\right\|_{L^{p}\left(\Omega^{1}\right)}$, we have

$$
\left(\int_{A} \varphi\left(\mathbf{Y}^{(h)}, \frac{\mathbf{z}_{p}}{d}\right) d \mathbf{z}-\int_{A} \varphi\left(\nabla_{p} \mathbf{y}^{(h)} \mid \frac{1}{h} \mathbf{y}_{, 3}^{(h)}, \frac{\mathbf{z}_{p}}{d}\right) d \mathbf{z}\right) \rightarrow 0 \quad \text { as } h \rightarrow 0 .
$$

Let $A_{z_{3}}$ be the projection of the slice of $A$ at the constant $z_{3}$, i.e.,

$$
A_{z_{3}}=\left\{\left(z_{1}, z_{2}\right):\left(z_{1}, z_{2}, z_{3}\right) \in A\right\} .
$$

Also, pick any $\hat{\mathbf{z}}_{p} \in P^{d}$ and let

$$
Q_{S}=\hat{\mathbf{z}}_{p}+d Z, \quad \text { and } Q=\left\{\left(\mathbf{z}_{p}, z_{3}\right): \mathbf{z}_{p} \in Q_{S}, \quad z_{3} \in(0,1)\right\},
$$

and notice that $\mathbf{Y}^{(h)}$ is constant over $Q_{S}$ for any fixed $z_{3} \in(0,1)$. Thus, using Fubini's theorem, we have

$$
\int_{A \cap Q} \varphi\left(\mathbf{Y}^{(h)}, \frac{\mathbf{z}_{p}}{d}\right) d \mathbf{z}=\int_{0}^{1} \int_{Q_{S} \cap A_{z_{3}}} \varphi\left(\mathbf{Y}^{(h)}, \frac{\mathbf{z}_{p}}{d}\right) d \mathbf{z}_{p} d z_{3}
$$

and

$$
\begin{aligned}
\int_{Q_{S} \cap A_{z_{3}}} \varphi\left(\mathbf{Y}^{(h)}, \frac{\mathbf{z}_{p}}{d}\right) d \mathbf{z}_{p} & =\int_{Q_{S}} \varphi\left(\mathbf{Y}^{(h)}, \frac{\mathbf{z}_{p}}{d}\right) d \mathbf{z}_{p}-\int_{Q_{S} \backslash A_{z_{3}}} \varphi\left(\mathbf{Y}^{(h)}, \frac{\mathbf{z}_{p}}{d}\right) d \mathbf{z}_{p} \\
& \geqq \int_{Q_{S}} \tilde{\varphi}\left(\mathbf{Y}^{(h)}\right) d \mathbf{z}_{p}-c_{2}\left(1+\left|\mathbf{Y}^{(h)}\right|^{p}\right)\left|Q_{S} \backslash A_{z_{3}}\right|
\end{aligned}
$$


where we have used the second inequality in (2.4). Using the other inequality in (2.4), we have

$$
\int_{Q_{S} \cap A_{z_{3}}} \varphi\left(\mathbf{Y}^{(h)}, \frac{\mathbf{z}_{p}}{d}\right) d \mathbf{z}_{p} \geqq c_{1}\left(\left|\mathbf{Y}^{(h)}\right|^{p}-1\right)\left|Q_{S} \cap A_{z_{3}}\right| .
$$

Combining (3.19) and (3.20), we obtain

$$
\int_{Q_{S} \cap A_{z_{3}}} \varphi\left(\mathbf{Y}^{(h)}, \frac{\mathbf{z}_{p}}{d}\right) d \mathbf{z}_{p} \geqq \int_{Q_{S}} \mu^{(d)}(\mathbf{z}) \tilde{\varphi}\left(\mathbf{Y}^{(h)}\right) d \mathbf{z}_{p}-2 c_{2} \int_{Q_{S} \backslash A_{z_{3}}} \mu^{(d)}(\mathbf{z}) d \mathbf{z}_{p},
$$

where

$$
\begin{aligned}
\mu^{(d)}(\mathbf{z}) & =\frac{c_{1}\left|Q_{S} \cap A_{z_{3}}\right|}{c_{1}\left|Q_{S} \cap A_{z_{3}}\right|+c_{2}\left|Q_{S} \backslash A_{z_{3}}\right|} \\
& =\frac{c_{1} f_{\hat{\mathbf{z}}_{p}+d Z} \chi_{A_{z_{3}}}\left(\tilde{\mathbf{z}}_{p}\right) d \tilde{\mathbf{z}}_{p}}{\left(c_{1}-c_{2}\right) f_{\hat{\mathbf{z}}_{p}+d Z} \chi_{A_{z_{3}}}\left(\tilde{\mathbf{z}}_{p}\right) d \tilde{\mathbf{z}}_{p}+c_{2}} \quad \text { for } \mathbf{z} \in Q
\end{aligned}
$$

and $\chi_{A_{3}}$ is the characteristic function of the set $A_{z_{3}}$. Integrating (3.21) over $z_{3}$ from 0 to 1 and summing the same equation over all $\hat{\mathbf{z}}_{p}$ in $P^{d}$, gives

$$
\int_{A} \varphi\left(\mathbf{Y}^{(h)}, \frac{\mathbf{z}_{p}}{d}\right) d \mathbf{z} \geqq \int_{\Omega^{1, d}} \mu^{(d)}(\mathbf{z}) \tilde{\varphi}\left(\mathbf{Y}^{(h)}\right) d \mathbf{z}-2 c_{2} \int_{\Omega^{1, d} \backslash A} \mu^{(d)}(\mathbf{z}) d \mathbf{z} .
$$

Invoking the Lebesgue point theorem on (3.22) as $d \rightarrow 0$ as $h \rightarrow 0$ for each fixed $z_{3}$, we have

$$
\begin{aligned}
\mu^{(d)}\left(\mathbf{z}_{p}, z_{3}\right) & \rightarrow \frac{c_{1} \chi_{A_{z_{3}}}\left(\mathbf{z}_{p}\right)}{c_{1} \chi_{A_{z_{3}}}\left(\mathbf{z}_{p}\right)+c_{2}\left(1-\chi_{A_{z_{3}}}\left(\mathbf{z}_{p}\right)\right)} \\
& =\chi_{A_{z_{3}}}\left(\mathbf{z}_{p}\right) \quad \text { a.e. on } \Omega^{\prime}
\end{aligned}
$$

and (3.23) becomes

$$
\liminf _{h \rightarrow 0} \int_{A} \varphi\left(\mathbf{Y}^{(h)}, \frac{\mathbf{z}_{p}}{d}\right) d \mathbf{z} \geqq \liminf _{h \rightarrow 0} \int_{A} \mu^{(d)}(\mathbf{z}) \tilde{\varphi}\left(\mathbf{Y}^{(h)}\right) d \mathbf{z} .
$$

Recalling (3.16), (3.25), (3.15), and the fact that $\varphi$ is nonnegative, we obtain

$$
\begin{aligned}
\liminf _{h \rightarrow 0} \int_{\Omega} \varphi\left(\nabla_{p} \mathbf{y}^{(h)} \mid \frac{1}{h} \mathbf{y}_{, 3}^{(h)}, \frac{\mathbf{z}_{p}}{d}\right) d \mathbf{z} & \geqq \liminf _{h \rightarrow 0} \int_{A} \varphi\left(\nabla_{p} \mathbf{y}^{(h)} \mid \frac{1}{h} \mathbf{y}_{, 3}^{(h)}, \frac{\mathbf{z}_{p}}{d}\right) d \mathbf{z} \\
& =\liminf _{h \rightarrow 0} \int_{A} \varphi\left(\mathbf{Y}^{(h)}, \frac{\mathbf{z}_{p}}{d}\right) d \mathbf{z} \\
& \geqq \liminf _{h \rightarrow 0} \int_{A} \mu^{(d)}(\mathbf{z}) \tilde{\varphi}\left(\mathbf{Y}^{(h)}\right) d \mathbf{z} \\
& =\liminf _{h \rightarrow 0} \int_{A} \mu^{(d)}(\mathbf{z}) \tilde{\varphi}\left(\nabla_{p} \mathbf{y}^{(h)} \mid \frac{1}{h} \mathbf{y}_{, 3}^{(h)}\right) d \mathbf{z} \\
& \geqq \liminf _{h \rightarrow 0} \int_{A} \mu^{(d)}(\mathbf{z}) \tilde{\varphi}_{0}\left(\nabla_{p} \mathbf{y}^{(h)}\right) d \mathbf{z .} .(3.26)
\end{aligned}
$$


Egoroff's theorem tells us that there exists a measurable subset $A^{\prime} \subset A$ with $\left|A \backslash A^{\prime}\right|<\delta^{\prime}$ such that for some subsequence (not relabeled)

$$
\mu^{(d)} \rightarrow \chi_{A_{z_{3}}} \equiv 1 \quad \text { uniformly on } \quad A^{\prime}
$$

as $d \rightarrow 0$ as $h \rightarrow 0$. Therefore, for any $\eta>0$, we have

$$
\begin{aligned}
\liminf _{h \rightarrow 0} \int_{A} \mu^{(d)}(\mathbf{z}) \tilde{\varphi}_{0}\left(\nabla_{p} \mathbf{y}^{(h)}\right) d \mathbf{z} & \geqq \liminf _{h \rightarrow 0} \int_{A^{\prime}} \mu^{(d)}(\mathbf{z}) \tilde{\varphi}_{0}\left(\nabla_{p} \mathbf{y}^{(h)}\right) d \mathbf{z} \\
& \geqq \liminf _{h \rightarrow 0} \int_{A^{\prime}}(1-\eta) Q \tilde{\varphi}_{0}\left(\nabla_{p} \mathbf{y}^{(h)}\right) d \mathbf{z} .
\end{aligned}
$$

If we define $G: W^{1, p}\left(\Omega^{1}, \mathbb{R}^{3}\right) \rightarrow \mathbb{R}$ by

$$
G(\hat{\mathbf{y}})=\int_{\Omega^{1}} Q \tilde{\varphi}_{0}\left(\nabla_{p} \hat{\mathbf{y}}\right) d \mathbf{z}
$$

and set $\Phi: \mathbb{M}^{3 \times 3} \rightarrow \mathbb{R}$ to be $\Phi\left(\mathbf{f}_{1}\left|\mathbf{f}_{2}\right| \mathbf{f}_{3}\right)=Q \tilde{\varphi}_{0}\left(\mathbf{f}_{1} \mid \mathbf{f}_{2}\right)$. Since $Q \tilde{\varphi}_{0}$ is quasiconvex, it can be shown [21] that $\Phi$ is also quasiconvex, bounded below by $-c_{1}$, and satisfies growth and coercivity conditions similar to (2.4). Then $G$ is sequentially lower semicontinuous on $W^{1, p}\left(\Omega^{1}, \mathbb{R}^{3}\right)$ (see ACERBI \& FUSCO [2]). Applying this result to $\int_{\Omega^{1}}(1-\eta) \chi_{A^{\prime}} Q \tilde{\varphi}_{0}\left(\nabla_{p} \mathbf{y}^{(h)}\right) d \mathbf{z}$, we have

$$
\liminf _{h \rightarrow 0} \int_{A^{\prime}}(1-\eta) Q \tilde{\varphi}_{0}\left(\nabla_{p} \mathbf{y}^{(h)}\right) d \mathbf{z} \geqq \int_{A^{\prime}}(1-\eta) Q \tilde{\varphi}_{0}\left(\nabla_{p} \mathbf{y}\right) d \mathbf{z}
$$

By letting $\delta^{\prime}$ and $\eta$ tend to zero, we have

$$
\liminf _{h \rightarrow 0} \int_{A} \mu^{(d)}(\mathbf{z}) \tilde{\varphi}_{0}\left(\nabla_{p} \mathbf{y}^{(h)}\right) d \mathbf{z} \geqq \int_{A} Q \tilde{\varphi}_{0}\left(\nabla_{p} \mathbf{y}\right) d \mathbf{z} .
$$

Combining this with (3.26) yields

$$
\liminf _{h \rightarrow 0} \int_{A} \varphi\left(\nabla_{p} \mathbf{y}^{(h)} \mid \frac{1}{h} \mathbf{y}_{, 3}^{(h)}, \frac{\mathbf{z}_{p}}{d}\right) d \mathbf{z} \geqq \int_{A} Q \tilde{\varphi}_{0}\left(\nabla_{p} \mathbf{y}\right) d \mathbf{z} .
$$

Using the fact that $Q \tilde{\varphi}_{0}\left(\nabla_{p} \mathbf{y}\right)$ belongs to $L^{1}\left(\Omega^{1}\right)$ and $\left|\Omega^{1} \backslash A\right|<2 \delta$, we obtain the desired lower bound by letting $\delta \rightarrow 0$.

We now consider case (ii): $\varphi=\varphi\left(\mathbf{F}, \frac{\mathbf{z}_{p}}{d}, z_{3}\right)$ and $\frac{\kappa}{d} \rightarrow \infty, \frac{\kappa}{h} \rightarrow \infty$ as $h \rightarrow 0$. We can construct the recovery sequence in a way similar to the previous case without any difficulty. The proof of the lower bound is also similar, except we have to replace (3.11) by

$$
\mathbf{Y}^{(h)}\left(\hat{\mathbf{z}}_{p}\right)=\frac{1}{d^{2}} \int_{0}^{1} \int_{\mathbf{z}_{p}+d Z}\left(\nabla_{p} \mathbf{y}^{(h)} \mid \frac{1}{h} \mathbf{y}_{, 3}^{(h)}\right) d \tilde{\mathbf{z}}_{p} d \tilde{z}_{3}, \quad \hat{\mathbf{z}}_{p} \in \mathbf{z}_{p}+d Z, \quad \mathbf{z}_{p} \in P^{d}
$$


Note that (3.12) remains valid, but (3.13) becomes

$$
\begin{aligned}
\| \mathbf{Y}^{(h)} & -\left(\nabla_{p} \mathbf{y}^{(h)} \mid \frac{1}{h} \mathbf{y}_{, 3}^{(h)}\right) \|_{L^{2}\left(\Omega^{1, d}\right)}^{2} \\
\leqq & C\left(\frac{d}{\kappa}\right)^{2} \int_{\Omega^{1, d}} \kappa^{2}\left(\left|\nabla_{p}^{2} \mathbf{y}^{(h)}\right|^{2}+\frac{1}{h^{2}}\left|\nabla_{p} \mathbf{y}_{, 3}^{(h)}\right|^{2}\right) d \mathbf{z} \\
& +C\left(\frac{h}{\kappa}\right)^{2} \int_{\Omega^{1, d}} \kappa^{2}\left(\frac{1}{h^{2}}\left|\nabla_{p} \mathbf{y}_{, 3}^{(h)}\right|^{2}+\frac{1}{h^{4}}\left|\mathbf{y}_{, 33}^{(h)}\right|^{2}\right) d \mathbf{z}
\end{aligned}
$$

where $C$ is some constant that does not depend on $h$. Since both $\frac{\kappa}{d} \rightarrow \infty, \frac{\kappa}{h} \rightarrow \infty$ as $h \rightarrow 0$, we obtain (3.14). The rest of the proof is similar and we omit it here.

Finally, case (iii) $\left(\varphi=\varphi\left(\mathbf{F}, z_{3}\right)\right.$ and $\frac{\kappa}{h} \rightarrow \infty$ as $\left.h \rightarrow 0\right)$ follows from case (ii).

Remark 3. In (3.6), we have assumed that $\mathbf{y}^{(\delta)}$ and $\mathbf{b}^{(\delta)}$ are smooth functions to allow the second derivative. Indeed, if $\mathbf{y}^{(\delta)} \in W^{1, p}\left(S, \mathbb{R}^{3}\right)$ and $\mathbf{b}^{(\delta)} \in L^{p}\left(S, \mathbb{R}^{3}\right)$, the bounded Lipschitz domain permits the existence of sequences $\mathbf{y}^{(\delta, \varepsilon)} \in C^{\infty}\left(\bar{S}, \mathbb{R}^{3}\right)$ and $\mathbf{b}^{(\delta, \varepsilon)} \in C_{0}^{\infty}\left(S, \mathbb{R}^{3}\right)$ such that

$$
\begin{aligned}
& \mathbf{y}^{(\delta, \varepsilon)} \rightarrow \mathbf{y}^{(\delta)} \quad \text { strongly in } W^{1, p}\left(S, \mathbb{R}^{3}\right) \\
& \mathbf{b}^{(\delta, \varepsilon)} \rightarrow \mathbf{b}^{(\delta)} \quad \text { strongly in } L^{p}\left(S, \mathbb{R}^{3}\right)
\end{aligned}
$$

as $\varepsilon \rightarrow 0$. Then, (3.6) is replaced by

$$
\mathbf{y}^{(\delta, \varepsilon, h)}(\mathbf{z})=\mathbf{y}^{(\delta, \varepsilon)}\left(\mathbf{z}_{p}\right)+h \mathbf{b}^{(\delta, \varepsilon)}\left(\mathbf{z}_{p}\right) z_{3}
$$

and (3.9) now becomes

$$
\limsup _{\delta \rightarrow 0} \limsup _{\varepsilon \rightarrow 0} \limsup _{h \rightarrow 0} e_{1}^{(h)}\left[\mathbf{y}^{(\delta, \varepsilon, h)}\right]=e_{1}^{(0)}[\mathbf{y}] .
$$

Appealing to the already quoted diagonalization argument, we find that there exists a recovery sequence labeled only in terms of $h$ and thus (3.10).

\section{Film thickness much smaller than heterogeneity}

Theorem 2. Suppose $\varphi=\varphi\left(\mathbf{F}, \frac{\mathbf{z}_{p}}{d}\right), \frac{\kappa}{d} \rightarrow \alpha$ and $\frac{h}{d} \rightarrow 0$ as $h \rightarrow 0$. Let $\overline{\mathbf{F}} \in \mathbb{M}^{3 \times 2}$, $Z=(0,1)^{2}$ and $e_{1}^{(h)}$ be defined by (2.6). Then, $e_{1}^{(h)} \Gamma$-converges to the functional $e_{1}^{(0)}$ defined by (2.7) if

(i) $\alpha=0$ and

$$
\begin{aligned}
& \bar{\varphi}(\overline{\mathbf{F}})=\inf _{k \in \mathbb{N}} \inf _{\boldsymbol{\omega} \in W_{0}^{1, p}(k Z)} f_{k Z} \varphi_{0}\left(\overline{\mathbf{F}}+\nabla_{p} \boldsymbol{\omega}, \mathbf{z}_{p}\right) d \mathbf{z}_{p}, \\
& \varphi_{0}\left(\overline{\mathbf{F}}^{0}, \mathbf{z}_{p}^{0}\right)=\inf _{\mathbf{b} \in \mathbb{R}^{3}} \varphi\left(\overline{\mathbf{F}}^{0} \mid \mathbf{b}, \mathbf{z}_{p}^{0}\right)
\end{aligned}
$$


(ii) $\alpha>0$ and

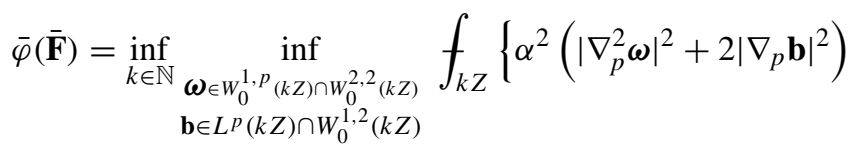

$$
\begin{aligned}
& \left.+\varphi\left(\overline{\mathbf{F}}+\nabla_{p} \omega \mid \mathbf{b}, \mathbf{z}_{p}\right)\right\} d \mathbf{z}_{p}
\end{aligned}
$$

(iii) $\alpha=\infty$ and

$$
\begin{gathered}
\bar{\varphi}(\overline{\mathbf{F}})=Q \tilde{\varphi}_{0}(\overline{\mathbf{F}}), \\
\tilde{\varphi}_{0}(\overline{\mathbf{F}})=\inf _{\mathbf{b} \in \mathbb{R}^{3}} \tilde{\varphi}(\overline{\mathbf{F}} \mid \mathbf{b}), \quad \tilde{\varphi}(\mathbf{F})=\int_{Z} \varphi\left(\mathbf{F}, \mathbf{z}_{p}\right) d \mathbf{z}_{p}
\end{gathered}
$$

where $Q \tilde{\varphi}_{0}$ is the lower quasi-convex envelope of $\tilde{\varphi}_{0}$.

Note that we have used the notation $f_{\Omega} \cdots=\frac{1}{|\Omega|} \int_{\Omega} \cdots$.

Remark 4. It can be shown that the effective energy density $\varphi$ satisfies the growth and Lipschitz condition for $0 \leqq \alpha \leqq \infty$, i.e.,

$$
\begin{aligned}
c_{1}\left(|\overline{\mathbf{F}}|^{p}-1\right) & \leqq \bar{\varphi}(\overline{\mathbf{F}}) \leqq c_{2}\left(|\overline{\mathbf{F}}|^{p}+1\right) \\
|\bar{\varphi}(\overline{\mathbf{F}})-\bar{\varphi}(\overline{\mathbf{G}})| & \leqq c_{2}^{\prime}\left(1+|\overline{\mathbf{F}}|^{p-1}+|\overline{\mathbf{G}}|^{p-1}\right)|\overline{\mathbf{F}}-\overline{\mathbf{G}}|
\end{aligned}
$$

for all $(\overline{\mathbf{F}}, \overline{\mathbf{G}}) \in \mathbb{M}^{3 \times 2} \times \mathbb{M}^{3 \times 2}$. Indeed, consider $0<\alpha<\infty$. The upper bound is obtained by setting $\boldsymbol{\omega}=\mathbf{b}=0$ in (4.3) and using (2.4) on $\varphi$. To show the lower bound, note that for every $\varepsilon>0$, there exists $k \in \mathbb{N}, \omega \in W_{0}^{1, p}\left(k Z, \mathbb{R}^{3}\right) \cap$ $W_{0}^{2,2}\left(k Z, \mathbb{R}^{3}\right)$ and $\mathbf{b} \in L^{p}\left(k Z, \mathbb{R}^{3}\right) \cap W_{0}^{1,2}\left(k Z, \mathbb{R}^{3}\right)$ such that

$$
\begin{aligned}
\bar{\varphi}(\overline{\mathbf{F}}) & \leqq f_{k Z}\left\{\alpha^{2}\left(\left|\nabla_{p}^{2} \boldsymbol{\omega}\right|^{2}+2\left|\nabla_{p} \mathbf{b}\right|^{2}\right)+\varphi\left(\overline{\mathbf{F}}+\nabla_{p} \omega \mid \mathbf{b}, \mathbf{z}_{p}\right)\right\} d \mathbf{z}_{p} \\
& \leqq \bar{\varphi}(\overline{\mathbf{F}})+\varepsilon
\end{aligned}
$$

Using (2.4) on $\varphi$ yields

$$
\begin{aligned}
\bar{\varphi}(\overline{\mathbf{F}})+\varepsilon & \geqq c_{1}\left(\left.f_{k Z}\left|\overline{\mathbf{F}}+\nabla_{p} \omega\right| \mathbf{b}\right|^{p} d \mathbf{z}_{p}-1\right) \\
& \geqq c_{1}\left(f_{k Z}\left|\overline{\mathbf{F}}+\nabla_{p} \omega\right|^{p} d \mathbf{z}_{p}-1\right) \\
& \geqq c_{1}\left(\left|f_{k Z}\left(\overline{\mathbf{F}}+\nabla_{p} \omega\right) d \mathbf{z}_{p}\right|^{p}-1\right) \\
& \geqq c_{1}\left(|\overline{\mathbf{F}}|^{p}-1\right)
\end{aligned}
$$


since $|\cdot|^{p}$ is convex and $\left.|\overline{\mathbf{F}}| \mathbf{b}\right|_{3 \times 3} \geqq|\overline{\mathbf{F}}|_{3 \times 2}$ for all $\mathbf{b} \in \mathbb{R}^{3}$. To prove the Lipschitz condition, we choose the same $\boldsymbol{\omega}$ and $\mathbf{b}$ as the test functions for $\bar{\varphi}(\overline{\mathbf{G}})$. We have

$$
\begin{aligned}
\bar{\varphi}(\overline{\mathbf{G}})-\bar{\varphi}(\overline{\mathbf{F}}) \leqq & f_{k Z}\left|\left(\varphi\left(\overline{\mathbf{G}}+\nabla_{p} \omega \mid \mathbf{b}, \mathbf{z}_{p}\right)-\varphi\left(\overline{\mathbf{F}}+\nabla_{p} \boldsymbol{\omega} \mid \mathbf{b}, \mathbf{z}_{p}\right)\right)\right| d \mathbf{z}_{p}+\varepsilon \\
\leqq & C\left(1+\frac{1}{k^{2}}\left\|\overline{\mathbf{G}}+\nabla_{p} \omega \mid \mathbf{b}\right\|_{L^{p}}^{p}\right. \\
& \left.\quad+\frac{1}{k^{2}}\left\|\overline{\mathbf{F}}+\nabla_{p} \boldsymbol{\omega} \mid \mathbf{b}\right\|_{L^{p}}^{p}\right)^{\frac{p-1}{p}}|\overline{\mathbf{G}}-\overline{\mathbf{F}}|+\varepsilon .
\end{aligned}
$$

Now invoking the growth conditions on $\varphi, \bar{\varphi}$ and (4.6), we get

$$
\begin{aligned}
C\left(\frac{1}{k^{2}}\left\|\overline{\mathbf{F}}+\nabla_{p} \omega \mid \mathbf{b}\right\|_{L^{p}}^{p}-1\right) & \leqq f \varphi\left(\overline{\mathbf{F}}+\nabla_{p} \omega \mid \mathbf{b}, \mathbf{z}_{p}\right) d \mathbf{z}_{p} \\
& \leqq \bar{\varphi}(\overline{\mathbf{F}})+\varepsilon \leqq c_{2}\left(|\overline{\mathbf{F}}|^{p}+1\right)+\varepsilon
\end{aligned}
$$

and

$$
\frac{1}{k^{2}}\left\|\overline{\mathbf{G}}+\nabla_{p} \boldsymbol{\omega} \mid \mathbf{b}\right\|_{L^{p}}^{p} \leqq C\left(|\overline{\mathbf{G}}-\overline{\mathbf{F}}|^{p}+\frac{1}{k^{2}}\left\|\overline{\mathbf{F}}+\nabla_{p} \boldsymbol{\omega} \mid \mathbf{b}\right\|_{L^{p}}^{p}\right) .
$$

Combining (4.7)-(4.9) gives us

$$
\bar{\varphi}(\overline{\mathbf{G}})-\bar{\varphi}(\overline{\mathbf{F}}) \leqq c_{2}^{\prime}\left(1+|\overline{\mathbf{F}}|^{p-1}+|\overline{\mathbf{G}}|^{p-1}\right)|\overline{\mathbf{F}}-\overline{\mathbf{G}}|+\varepsilon .
$$

We have the desired inequality as $\varepsilon \rightarrow 0$. The opposite inequality can be obtained by interchanging $\overline{\mathbf{F}}$ and $\overline{\mathbf{G}}$. The case $\alpha=0$ and $\alpha=\infty$ can be treated similarly.

Proof of Theorem 2. The case $\alpha=\infty$ is a corollary of Theorem 1. The proof for finite $\alpha \geqq 0$ consists of two parts. First, we prove the $\Gamma$-limit in the case where the limit function is affine (Part A). We then prove the general case by approximating an arbitrary function by piecewise affine functions (Part B).

Part A. Suppose $\mathbf{y}=\overline{\mathbf{F}} \mathbf{z}_{p}$ with $\overline{\mathbf{F}} \in \mathbb{M}^{3 \times 2}$. We begin by constructing a recovery sequence for the case where $\alpha>0$. It follows from the definition of $\bar{\varphi}$ that there exist sequences $k^{(\varepsilon)} \in \mathbb{N}, \omega^{(\varepsilon)} \in W_{0}^{1, p}\left(k^{(\varepsilon)} Z, \mathbb{R}^{3}\right) \cap W_{0}^{2,2}\left(k^{(\varepsilon)} Z, \mathbb{R}^{3}\right)$, and $\mathbf{b}^{(\varepsilon)} \in$ $L^{p}\left(k^{(\varepsilon)} Z, \mathbb{R}^{3}\right) \cap W_{0}^{1,2}\left(k^{(\varepsilon)} Z, \mathbb{R}^{3}\right)$ such that

$$
\begin{aligned}
& \frac{1}{k^{(\varepsilon) 2}} \int_{k^{(\varepsilon)} Z}\left\{\alpha^{2}\left(\left|\nabla_{p}^{2} \boldsymbol{\omega}^{(\varepsilon)}\right|^{2}+2\left|\nabla_{p} \mathbf{b}^{(\varepsilon)}\right|^{2}\right)+\varphi\left(\overline{\mathbf{F}}+\nabla_{p} \omega^{(\varepsilon)} \mid \mathbf{b}^{(\varepsilon)}, \mathbf{z}_{p}\right)\right\} d \mathbf{z}_{p} \\
& \rightarrow \bar{\varphi}(\overline{\mathbf{F}})
\end{aligned}
$$

as $\varepsilon \rightarrow 0$. We use $\boldsymbol{\omega}^{(\varepsilon)}$ and $\mathbf{b}^{(\varepsilon)}$ to construct our recovery sequence. Unfortunately, $\mathbf{b}^{(\varepsilon)}$ may not be smooth enough to allow second differentiation. However, an approximation argument similar to Remark 3 shows that we may assume that $\mathbf{b}^{(\varepsilon)} \in C_{0}^{\infty}\left(k^{(\varepsilon)} Z, \mathbb{R}^{3}\right)$. Define

$$
\mathbf{y}^{(h, \varepsilon)}=\overline{\mathbf{F}} \mathbf{z}_{p}+d \boldsymbol{\omega}^{(\varepsilon)}\left(\frac{\mathbf{z}_{p}}{d}\right)+h \mathbf{b}^{(\varepsilon)}\left(\frac{\mathbf{z}_{p}}{d}\right) z_{3},
$$


where $\boldsymbol{\omega}^{(\varepsilon)}$ and $\mathbf{b}^{(\varepsilon)}$ are extended periodically in the plane of the film since $\boldsymbol{\omega}^{(\varepsilon)}=$ $\partial \boldsymbol{\omega}^{(\varepsilon)} / \partial n=\mathbf{b}^{(\varepsilon)}=\partial \mathbf{b}^{(\varepsilon)} / \partial n=0$ on $\partial k^{(\varepsilon)} Z$ in the sense of trace. Clearly, $\mathbf{y}^{(h, \varepsilon)} \stackrel{h}{\rightarrow}$ $\overline{\mathbf{F}} \mathbf{z}_{p}$ for each $\varepsilon>0$. Substituting (4.12) into $e_{1}^{(h)}$ defined by (2.6), we have

$$
\begin{aligned}
e_{1}^{(h)}[\mathbf{y}(h, \varepsilon)]=\int_{\Omega^{1}} & \left\{\left(\left|\frac{\kappa}{d} \nabla_{p}^{2} \boldsymbol{\omega}^{(\varepsilon)}+\frac{h}{d} \cdot \frac{\kappa}{d} \nabla_{p}^{2} \mathbf{b}^{(\varepsilon)} z_{3}\right|^{2}+2\left|\frac{\kappa}{d} \nabla_{p} \mathbf{b}^{(\varepsilon)}\right|^{2}\right)\right. \\
& \left.+\varphi\left(\overline{\mathbf{F}}+\nabla_{p} \boldsymbol{\omega}^{(\varepsilon)}+\frac{h}{d} \nabla_{p} \mathbf{b} z_{3} \mid \mathbf{b}^{(\varepsilon)}, \frac{\mathbf{z}_{p}}{d}\right)\right\} d \mathbf{z} .
\end{aligned}
$$

If we assume that $\frac{h}{d} \rightarrow 0$ and $\frac{\kappa}{d} \rightarrow \alpha$ as $h \rightarrow 0$, and impose the Lipschitz condition (2.5) on $\varphi$, we get

$$
\begin{aligned}
& e_{1}^{(h)}\left[\mathbf{y}^{(h, \varepsilon)}\right] \rightarrow \frac{\left|\Omega^{1}\right|}{k^{(\varepsilon) 2}} \int_{k^{(\varepsilon)} Z}\{ \alpha^{2}\left(\left|\nabla_{p}^{2} \boldsymbol{\omega}^{(\varepsilon)}\right|^{2}+2\left|\nabla_{p} \mathbf{b}^{(\varepsilon)}\right|^{2}\right) \\
&\left.+\varphi\left(\overline{\mathbf{F}}+\nabla_{p} \boldsymbol{\omega}^{(\varepsilon)} \mid \mathbf{b}^{(\varepsilon)}, \mathbf{z}_{p}\right)\right\} d \mathbf{z}_{p}
\end{aligned}
$$

as $h \rightarrow 0$. Above, we have applied the property of mean value ${ }^{2}$ to (4.13) in deriving (4.14). Then, using (4.11) gives

$$
\limsup _{\varepsilon \rightarrow 0} \limsup _{h \rightarrow 0} e_{1}^{(h)}\left[\mathbf{y}^{(h, \varepsilon)}\right]=\left|\Omega^{1}\right| \bar{\varphi}(\overline{\mathbf{F}})=|S| \bar{\varphi}(\overline{\mathbf{F}})=e_{1}^{(0)}[\mathbf{y}] .
$$

Recalling (4.12) and (4.15) and appealing to the standard diagonalization argument, we find that there exists a sequence $\hat{\mathbf{y}}^{(h)}=\mathbf{y}^{(h, \varepsilon(h))}$ that converges weakly to $\mathbf{y}=\overline{\mathbf{F}} \mathbf{z}_{p}$ in $W^{1, p}\left(\Omega^{1}, \mathbb{R}^{3}\right)$ and satisfies

$$
\lim _{h \rightarrow 0} e_{1}^{(h)}\left[\hat{\mathbf{y}}^{(h)}\right]=e_{1}^{(0)}[\mathbf{y}] .
$$

The case of $\alpha=0$ is similar. Indeed, an argument similar to that in Remark 2 shows that (4.2) is well defined and the infimum is also achieved. From the definition of $\bar{\varphi}$, there exists sequences $k^{(\varepsilon)} \in \mathbb{N}, \omega^{(\varepsilon)} \in W_{0}^{1, p}\left(k^{(\varepsilon)} Z, \mathbb{R}^{3}\right)$ such that

$$
\frac{1}{k^{(\varepsilon) 2}} \int_{k^{(\varepsilon)} Z} \varphi_{0}\left(\overline{\mathbf{F}}+\nabla_{p} \boldsymbol{\omega}^{(\varepsilon)}, \mathbf{z}_{p}\right) d \mathbf{z}_{p} \rightarrow \bar{\varphi}(\overline{\mathbf{F}})
$$

as $\varepsilon \rightarrow 0$. Following an argument like the one used in (3.5), we find measurable functions $\mathbf{b}^{(\varepsilon)} \in L^{p}\left(k^{(\varepsilon)} Z, \mathbb{R}^{3}\right)$ such that

$$
\varphi_{0}\left(\overline{\mathbf{F}}+\nabla_{p} \boldsymbol{\omega}^{(\varepsilon)}, \mathbf{z}_{p}\right)=\varphi\left(\overline{\mathbf{F}}+\nabla_{p} \boldsymbol{\omega}^{(\varepsilon)} \mid \mathbf{b}^{(\varepsilon)}, \mathbf{z}_{p}\right)
$$

for almost all $\mathbf{z}_{p} \in k^{(\varepsilon)} Z$. The rest of the proof follows similarly.

2 ibid 1. 
Set $\boldsymbol{\omega}^{(h)}=\mathbf{y}^{(h)}-\overline{\mathbf{F}} \mathbf{z}_{p}$. A slight refinement (see Lemma 2.1 in MüLlER [36]) shows that the recovery sequence $\mathbf{y}^{(h)}$ can be constructed such that

$$
\boldsymbol{\omega}^{(h)}=\frac{\partial \boldsymbol{\omega}^{(h)}}{\partial n}=0
$$

on $\Sigma=\partial S \times(0,1)$ in the sense of trace.

We now turn to the lower bound when $\mathbf{y}$ is an affine function and $\alpha>0$. We assume that $S$ is a square domain with side length $s$ and

$$
\begin{aligned}
& \boldsymbol{\omega}^{(h)}=\mathbf{y}^{(h)}-\overline{\mathbf{F}} \mathbf{z}_{p} \stackrel{h}{\rightarrow} 0 \quad \text { in } W^{1, p}\left(\Omega^{1}, \mathbb{R}^{3}\right), \\
& \boldsymbol{\omega}^{(h)}=\frac{\partial \boldsymbol{\omega}^{(h)}}{\partial n}=0
\end{aligned}
$$

on $\Sigma=\partial S \times(0,1)$ in the sense of trace. We may also assume that $\lim _{\inf }{ }_{h \rightarrow 0} e_{1}^{(h)}$ $\left[\mathbf{y}^{(h)}\right]$ is finite; otherwise the proof is trivial. Choose $k \in \mathbb{N}$ to be smallest integer such that $k d \geqq s+d$. We can find a square $\tilde{S}^{(d)}$ with the side length $k d$ such that $S \subset \tilde{S}^{(d)}$ and the corners of $\tilde{S}^{(d)}$ are in $d \mathbb{Z}^{2}$, i.e., $\tilde{S}^{(d)}=d\left(\mathbf{z}_{p}^{0}+k Z\right)$ for some $\mathbf{z}_{p}^{0} \in \mathbb{Z}^{2}$. Now extending $\omega^{(h)}$ to $\tilde{S}^{(d)} \times(0,1)$ by

$$
\tilde{\boldsymbol{\omega}}^{(h)}= \begin{cases}\boldsymbol{\omega}^{(h)}, & \text { for } \quad \mathbf{z} \in S \times(0,1), \\ 0, & \text { for } \quad \mathbf{z} \in\left(\tilde{S}^{(d)} \backslash S\right) \times(0,1),\end{cases}
$$

we have

$$
\begin{aligned}
e_{1}^{(h)}\left[\mathbf{y}^{(h)}\right]= & \int_{\tilde{S}^{(d)} \times(0,1)}\left\{\kappa^{2}\left(\left|\nabla_{p}^{2} \tilde{\boldsymbol{\omega}}^{(h)}\right|^{2}+\frac{2}{h^{2}}\left|\nabla_{p} \tilde{\boldsymbol{\omega}}_{, 3}^{(h)}\right|^{2}+\frac{1}{h^{4}}\left|\tilde{\boldsymbol{\omega}}_{, 33}^{(h)}\right|^{2}\right)\right. \\
& \left.+\varphi\left(\overline{\mathbf{F}}+\nabla_{p} \tilde{\boldsymbol{\omega}}^{(h)} \mid \frac{1}{h} \tilde{\boldsymbol{\omega}}_{, 3}, \frac{\mathbf{z}_{p}}{d}\right)\right\} d \mathbf{z} \\
& -\int_{\left(\tilde{S}^{(d)} \backslash S\right) \times(0,1)} \varphi\left(\overline{\mathbf{F}} \mid 0, \frac{\mathbf{z}_{p}}{d}\right) d \mathbf{z} \\
= & I_{1}-I_{2} .
\end{aligned}
$$

The second integral $-I_{2} \geqq-c_{2}\left(1+|\overline{\mathbf{F}}|^{p}\right)\left|\tilde{S}^{(d)} \backslash S\right|$ converges to zero since $\left|\tilde{S}^{(d)} \backslash S\right| \leqq(s+2 d)^{2}-s^{2} \rightarrow 0$ as $d$ tends to zero as $h$ tends to zero. Changing variables $\mathbf{z}_{p} \mapsto d\left(\mathbf{z}_{p}^{0}+\hat{\mathbf{z}}_{p}\right)$ and $z_{3} \mapsto \frac{d}{h} \hat{z}_{3}$, using the periodicity of $\varphi$ and Fubini's theorem, we have

$$
\begin{aligned}
I_{1} \geqq d^{2} \cdot \frac{d}{h} \cdot \int_{0}^{\frac{h}{d}} \int_{k Z}\{ & \alpha^{2}\left(\left|\nabla_{p}^{2} \hat{\boldsymbol{\omega}}^{(h)}\right|^{2}+2\left|\nabla_{p} \hat{\boldsymbol{\omega}}_{, 3}^{(h)}\right|^{2}\right) \\
& \left.+\varphi\left(\overline{\mathbf{F}}+\nabla_{p} \hat{\boldsymbol{\omega}}^{(h)} \mid \hat{\boldsymbol{\omega}}_{, 3}^{(h)}, \hat{\mathbf{z}}_{p}\right)\right\} d \hat{\mathbf{z}}_{p} d \hat{z}_{3}
\end{aligned}
$$

where

$$
\hat{\boldsymbol{\omega}}^{(h)}\left(\hat{\mathbf{z}}_{p}, \hat{z}_{3}\right)=\frac{1}{d} \tilde{\boldsymbol{\omega}}^{(h)}\left(d \hat{\mathbf{z}}_{p}, \frac{d}{h} \hat{z}_{3}\right) .
$$


Notice that for almost every $\hat{z}_{3} \in\left(0, \frac{h}{d}\right)$, the function $\hat{\boldsymbol{\omega}}_{\hat{z}_{3}}^{(h)}\left(\hat{\mathbf{z}}_{p}\right)=\hat{\boldsymbol{\omega}}^{(h)}\left(\hat{\mathbf{z}}_{p}, \hat{z}_{3}\right)$ belongs to $W_{0}^{1, p}\left(k Z, \mathbb{R}^{3}\right) \cap W_{0}^{2,2}\left(k Z, \mathbb{R}^{3}\right)$. Similarly, for almost every $\hat{z}_{3} \in\left(0, \frac{h}{d}\right)$, $\hat{\boldsymbol{\omega}}_{, 3}^{(h)}$ belongs to $L^{p}\left(k Z, \mathbb{R}^{3}\right) \cap W_{0}^{1,2}\left(k Z, \mathbb{R}^{3}\right)$. It follows that

$$
I_{1} \geqq d^{2} \cdot \frac{d}{h} \cdot \int_{0}^{\frac{h}{d}} k^{2} \bar{\varphi}(\overline{\mathbf{F}}) d z_{3} \geqq(d k)^{2} \bar{\varphi}(\overline{\mathbf{F}}) \geqq s^{2} \bar{\varphi}(\overline{\mathbf{F}}) .
$$

Thus, we have shown that $\liminf _{h \rightarrow 0} e_{1}^{(h)}\left[\mathbf{y}^{(h)}\right] \geqq s^{2} \bar{\varphi}(\overline{\mathbf{F}})=e_{1}^{(0)}[\mathbf{y}]$ which is the desired lower bound.

For the general domain $S$, assume the sequence $\mathbf{y}^{(h)}$ satisfies (4.18). Consider a square $Q$ which contains $S$. Using the fact that the recovery sequence can be obtained such that (4.17) is satisfied for the domain $(Q \backslash S) \times(0,1)$, we can also obtain the lower bound.

Now let $S$ be any open bounded Lipschitz domain and let $\mathbf{y}^{(h)} \stackrel{h}{\rightarrow} \mathbf{y}=\overline{\mathbf{F}} \mathbf{z}_{p}$ in $W^{1, p}\left(\Omega^{1}, \mathbb{R}^{3}\right)$. No further assumption such as (4.18) is imposed on $\mathbf{y}^{(h)}$. We use the argument of De Giorgi [25] (see also Francfort \& Müller [24]) to obtain the lower bound. Fix $S_{0}$ open and compactly contained in $S$. Let

$$
R=\frac{1}{2} \operatorname{dist}\left(S_{0}, \partial S\right) .
$$

For any strictly positive integer $v$, define

$$
S_{i}=\left\{\mathbf{z}_{p} \in S: \operatorname{dist}\left(\mathbf{z}_{p}, S_{0}\right)<\frac{i}{v} R\right\}, \quad 1 \leqq i \leqq v,
$$

and scalar functions $\eta_{i}\left(\mathbf{z}_{p}\right) \in C_{0}^{\infty}(S)$ such that

$$
\begin{cases}0 \leqq \eta_{i} \leqq 1, & \\ \eta_{i}=1 \quad \text { in } \quad S_{i-1} & \text { and } \eta_{i}=0 \text { in } S \backslash S_{i}, \\ \left|\nabla_{p} \eta_{i}\right| \leqq \frac{v+1}{R} & \text { and }\left|\nabla_{p}^{2} \eta_{i}\right| \leqq\left(\frac{v+1}{R}\right)^{2} .\end{cases}
$$

Moreover, let $\Omega_{i}^{1}=S_{i} \times(0,1)$ for $i=0, \cdots, v$ and set

$$
\mathbf{y}_{i}^{(h)}=\overline{\mathbf{F}} \mathbf{z}_{p}+\eta_{i}\left(\mathbf{y}^{(h)}-\overline{\mathbf{F}} \mathbf{z}_{p}\right) .
$$

Then, for each $i, \mathbf{y}_{i}^{(h)}$ converges weakly to $\mathbf{y}=\overline{\mathbf{F}} \mathbf{z}_{p}$ in $W^{1, p}\left(\Omega^{1}, \mathbb{R}^{3}\right)$ as $h$ tends to zero and $\left(\mathbf{y}_{i}^{(h)}-\overline{\mathbf{F}} \mathbf{z}_{p}\right)$ satisfies (4.18). Therefore, it follows from the previous result that

$$
\liminf _{h \rightarrow 0} e_{1}^{(h)}\left[\mathbf{y}_{i}^{(h)} ; \Omega^{1}\right] \geqq e_{1}^{(0)}\left[\mathbf{y} ; \Omega^{1}\right] .
$$

Now

$$
\begin{aligned}
e_{1}^{(h)}\left[\mathbf{y}_{i}^{(h)} ; \Omega^{1}\right]= & e_{1}^{(h)}\left[\mathbf{y}^{(h)} ; \Omega_{i-1}^{1}\right]+e_{1}^{(h)}\left[\mathbf{y}_{i}^{(h)} ; \Omega_{i}^{1} \backslash \Omega_{i-1}^{1}\right]+e_{1}^{(h)}\left[\mathbf{y} ; \Omega^{1} \backslash \Omega_{i}^{1}\right] \\
\leqq & e_{1}^{(h)}\left[\mathbf{y}^{(h)} ; \Omega^{1}\right]+e_{1}^{(h)}\left[\mathbf{y}_{i}^{(h)} ; \Omega_{i}^{1} \backslash \Omega_{i-1}^{1}\right] \\
& +c_{2}\left(1+|\overline{\mathbf{F}}|^{p}\right)\left|\Omega^{1} \backslash \Omega_{0}^{1}\right| .
\end{aligned}
$$


Using the growth condition (2.4) on $\varphi$ and the definition of $\eta_{i}\left(\mathbf{z}_{p}\right)$,

$$
\begin{aligned}
e_{1}^{(h)}\left[\mathbf{y}_{i}^{(h)} ; \Omega_{i}^{1} \backslash \Omega_{i-1}^{1}\right] & \\
\leqq C \int_{\Omega_{i}^{1} \backslash \Omega_{i-1}^{1}} & \left\{\kappa ^ { 2 } \left(\left|\nabla_{p}^{2} \mathbf{y}^{(h)}\right|^{2}+\frac{2}{h^{2}}\left|\nabla_{p} \mathbf{y}_{, 3}^{(h)}\right|^{2}+\frac{1}{h^{4}}\left|\mathbf{y}_{, 33}^{(h)}\right|^{2}\right.\right. \\
+ & \left(\frac{v+1}{R}\right)^{4}\left|\mathbf{y}^{(h)}-\overline{\mathbf{F}} \mathbf{z}_{p}\right|^{2}+4\left(\frac{v+1}{R}\right)^{2}\left|\nabla_{p} \mathbf{y}^{(h)}-\overline{\mathbf{F}}\right|^{2} \\
+ & \left.\frac{2}{h^{2}}\left(\frac{v+1}{R}\right)^{2}\left|\mathbf{y}_{, 3}^{(h)}\right|^{2}\right) \\
+ & \left(1+|\overline{\mathbf{F}}|^{p}+\left|\nabla_{p} \mathbf{y}^{(h)}-\overline{\mathbf{F}}\right|^{p}+\left(\frac{v+1}{R}\right)^{p} \mid \mathbf{y}^{(h)}\right. \\
& \left.\left.-\left.\overline{\mathbf{F}} \mathbf{z}_{p}\right|^{p}+\left|\frac{1}{h} \mathbf{y}_{, 3}^{(h)}\right|^{p}\right)\right\} d \mathbf{z} .
\end{aligned}
$$

Notice that we have used the inequality $\left(\sum_{i=1}^{N}\left|a_{i}\right|\right)^{2} \leqq N \sum_{i=1}^{N}\left|a_{i}\right|^{2}$ in deriving (4.23). Since $\mathbf{y}^{(h)} \stackrel{h}{\rightarrow} \overline{\mathbf{F}} \mathbf{z}_{p}$ in $W^{1, p}\left(\Omega^{1}, \mathbb{R}^{3}\right)$, this implies $\mathbf{y}^{(h)} \stackrel{h}{\rightarrow} \overline{\mathbf{F}} \mathbf{z}_{p}$ in $L^{p}\left(\Omega^{1}\right.$; $\mathbb{R}^{3}$ ) by Rellich's compactness theorem and

$$
\int_{\Omega_{i}^{1} \backslash \Omega_{i-1}^{1}}\left(\frac{v+1}{R}\right)^{p}\left|\mathbf{y}^{(h)}-\overline{\mathbf{F}} \mathbf{z}_{p}\right|^{p} d \mathbf{z} \rightarrow 0 \quad \text { as } h \rightarrow 0 .
$$

By the assumptions of finiteness of $\liminf _{h \rightarrow 0} e_{1}^{(h)}\left[\mathbf{y}^{(h)}\right]$ and non-negativity of $\varphi$, it is concluded that $\left\|\frac{\kappa}{h} \nabla \mathbf{y}_{, 3}^{(h)}\right\|_{L^{2}\left(\Omega^{1}\right)}$ is uniformly bounded in $h$. Further since

$$
\left|\int_{\Omega^{1}} \frac{\kappa}{h} \mathbf{y}_{, 3}^{(h)} d \mathbf{z}\right| \leqq C \kappa\left\|\frac{1}{h} \mathbf{y}_{, 3}^{(h)}\right\|_{L^{p}\left(\Omega^{1}\right)},
$$

and $\frac{1}{h} \mathbf{y}_{3}^{(h)}$ is uniformly bounded in $L^{p}\left(\Omega^{1} ; \mathbb{R}^{3}\right)$ due to coercivity of $\varphi$, the Poincaré inequality implies

$$
\frac{\kappa}{h} \mathbf{y}_{, 3}^{(h)} \rightarrow 0 \quad \text { in } W^{1,2}\left(\Omega^{1} ; \mathbb{R}^{3}\right) \quad \text { as } h \rightarrow 0 .
$$

Similarly, $\left\|\kappa \nabla^{2} \mathbf{y}^{(h)}\right\|_{L^{2}\left(\Omega^{1}\right)}$ is uniformly bounded in $h$. Using the Poincaré inequality twice implies that

$$
\kappa \mathbf{y}^{(h)} \rightarrow 0 \quad \text { in } W^{2,2}\left(\Omega^{1} ; \mathbb{R}^{3}\right) \quad \text { as } h \rightarrow 0,
$$

from which it is deduced that

$$
\int_{\Omega_{i}^{1} \backslash \Omega_{i-1}^{1}} \kappa^{2}\left\{\left|\mathbf{y}^{(h)}-\overline{\mathbf{F}} \mathbf{z}_{p}\right|^{2}+\left|\nabla_{p} \mathbf{y}^{(h)}-\overline{\mathbf{F}}\right|^{2}+\left|\frac{1}{h} \mathbf{y}_{, 3}^{(h)}\right|^{2}\right\} d \mathbf{z} \rightarrow 0,
$$


as $h \rightarrow 0$. Collecting (4.21) to (4.25) gives

$$
\begin{aligned}
e_{1}^{(0)}[\mathbf{y}] \leqq & \liminf _{h \rightarrow 0} e_{1}^{(h)}\left[\mathbf{y}^{(h)}\right]+c_{2}\left(1+|\overline{\mathbf{F}}|^{p}\right)\left|\Omega^{1} \backslash \Omega_{0}^{1}\right| \\
& +C \int_{\Omega_{i}^{1} \backslash \Omega_{i-1}^{1}}\left\{\kappa^{2}\left(\left|\nabla_{p}^{2} \mathbf{y}^{(h)}\right|^{2}+\frac{2}{h^{2}}\left|\nabla_{p} \mathbf{y}_{, 3}^{(h)}\right|^{2}+\frac{1}{h^{4}}\left|\mathbf{y}_{, 33}^{(h)}\right|^{2}\right)\right. \\
& \left.+\left(1+|\overline{\mathbf{F}}|^{p}+\left|\nabla_{p} \mathbf{y}^{(h)}-\overline{\mathbf{F}}\right|^{p}+\left|\frac{1}{h} \mathbf{y}_{, 3}^{(h)}\right|^{p}\right)\right\} d \mathbf{z} .
\end{aligned}
$$

Summing (4.26) over $i=1, \cdots, v$ and dividing by $v$ gives

$$
\begin{aligned}
e_{1}^{(0)}[\mathbf{y}] \leqq & \liminf _{h \rightarrow 0} e_{1}^{(h)}\left[\mathbf{y}^{(h)}\right]+c_{2}\left(1+|\overline{\mathbf{F}}|^{p}\right)\left|\Omega^{1} \backslash \Omega_{0}^{1}\right| \\
& +\frac{C}{v} \int_{\Omega^{1}}\left\{\kappa^{2}\left(\left|\nabla_{p}^{2} \mathbf{y}^{(h)}\right|^{2}+\frac{2}{h^{2}}\left|\nabla_{p} \mathbf{y}_{, 3}^{(h)}\right|^{2}+\frac{1}{h^{4}}\left|\mathbf{y}_{, 33}^{(h)}\right|^{2}\right)\right. \\
& \left.\quad+\left(1+|\overline{\mathbf{F}}|^{p}+\left|\nabla_{p} \mathbf{y}^{(h)}-\overline{\mathbf{F}}\right|^{p}+\left|\frac{1}{h} \mathbf{y}_{, 3}^{(h)}\right|^{p}\right)\right\} d \mathbf{z} .
\end{aligned}
$$

Recall the assumption of finiteness of $\liminf _{h \rightarrow 0} e_{1}^{(h)}\left[\mathbf{y}^{(h)}\right]$ and note that $\| \nabla_{p} \mathbf{y}^{(h)}-$ $\overline{\mathbf{F}} \|_{L^{p}\left(\Omega^{1}\right)}$ and $\left\|\frac{1}{h} \mathbf{y}_{, 3}^{(h)}\right\|_{L^{p}\left(\Omega^{1}\right)}$ are uniformly bounded in $h$ since $\mathbf{y}^{(h)} \rightarrow \overline{\mathbf{F}} \mathbf{z}_{p}$ in $W^{1, p}\left(\Omega^{1}, \mathbb{R}^{3}\right)$ and $\varphi$ enjoys the coercivity (2.4). This concludes the proof by letting $\nu \rightarrow+\infty$ and $\Omega_{0}^{1} \rightarrow \Omega^{1}$.

The proof for the case where $\alpha=0$ is almost exactly the same except we use

$$
\varphi\left(\overline{\mathbf{F}}+\nabla_{p} \tilde{\boldsymbol{\omega}}^{(h)} \mid \frac{1}{h} \tilde{\boldsymbol{\omega}}_{, 3}, \frac{\mathbf{z}_{p}}{d}\right) \geqq \varphi_{0}\left(\overline{\mathbf{F}}+\nabla_{p} \tilde{\boldsymbol{\omega}}^{(h)}, \frac{\mathbf{z}_{p}}{d}\right)
$$

after (4.19).

Part B. In the case where $\mathbf{y}$ is the piecewise affine function, the proof for the lower bound is obvious. The recovery sequence can also be constructed by virtue of (4.17).

For general $\mathbf{y} \in V_{S}$, the existence of a recovery sequence can be deduced as follows. The Lipschitz boundary $\partial S$ of the film guarantees the existence of a sequence of piecewise affine functions $\mathbf{y}^{(\delta)}$ such that

$$
\mathbf{y}^{(\delta)} \rightarrow \mathbf{y} \quad \text { in } W^{1, p}\left(S, \mathbb{R}^{3}\right) \quad \text { as } \delta \rightarrow 0
$$

For each piecewise affine function $\mathbf{y}^{(\delta)}$, there exists a recovery sequence $\mathbf{y}^{(h, \delta)}$ such that

$$
\mathbf{y}^{(h, \delta)} \rightarrow \mathbf{y}^{(\delta)} \quad \text { in } W^{1, p}\left(\Omega^{1}, \mathbb{R}^{3}\right) \quad \text { and } e_{1}^{(h)}\left[\mathbf{y}^{(h, \delta)}\right] \rightarrow e_{1}^{(0)}\left[\mathbf{y}^{(\delta)}\right]
$$

as $h \rightarrow 0$. Define

$$
\begin{aligned}
f(h, \delta)= & \left|e_{1}^{(h)}\left[\mathbf{y}^{(h, \delta)}\right]-e_{1}^{(0)}[\mathbf{y}]\right|+\left\|\mathbf{y}^{(h, \delta)}-\mathbf{y}\right\|_{L^{p}\left(\Omega^{1}\right)} \\
\leqq & \left|e_{1}^{(h)}\left[\mathbf{y}^{(h, \delta)}\right]-e_{1}^{(0)}\left[\mathbf{y}^{(\delta)}\right]\right|+\left|e_{1}^{(0)}\left[\mathbf{y}^{(\delta)}\right]-e_{1}^{(0)}[\mathbf{y}]\right| \\
& +\left\|\mathbf{y}^{(h, \delta)}-\mathbf{y}^{(\delta)}\right\|_{L^{p}\left(\Omega^{1}\right)}+\left\|\mathbf{y}^{(\delta)}-\mathbf{y}\right\|_{L^{p}\left(\Omega^{1}\right)}
\end{aligned}
$$


The Lipschitz condition of (4.5) for the homogenized energy density $\bar{\varphi}$ implies that

$$
\left|e_{1}^{(0)}\left[\mathbf{y}^{(\delta)}\right]-e_{1}^{(0)}[\mathbf{y}]\right| \rightarrow 0 \quad \text { as } \delta \rightarrow 0 .
$$

It follows that $\lim \sup _{\delta \rightarrow 0} \limsup _{h \rightarrow 0} f(h, \delta)=0$. A standard diagonalization argument establishes the existence of a recovery sequence.

It remains to prove the lower bound for general $\mathbf{y} \in V_{S}$. Let $\mathbf{y}^{(h)} \rightarrow \mathbf{y}$ in $W^{1, p}\left(\Omega^{1}, \mathbb{R}^{3}\right)$ as $h \rightarrow 0$. Without loss of generality, we may assume that $\lim \inf _{h \rightarrow 0} e_{1}^{(h)}\left[\mathbf{y}^{(h)}\right]$ is finite. First, the regularity of $\partial S$ permits the existence of a sequence $\omega^{(h)} \in C^{\infty}(\bar{S})$ such that

$$
\begin{aligned}
\boldsymbol{\omega}^{(h)} \rightarrow \mathbf{y} & \text { in } W^{1, p}\left(S, \mathbb{R}^{3}\right), \\
\kappa \nabla_{p}^{2} \boldsymbol{\omega}^{(h)} \rightarrow 0 & \text { in } L^{2}\left(S, \mathbb{R}^{12}\right)
\end{aligned}
$$

as $h \rightarrow 0$ (cf. FranCFORT \& MüLler [24]). For any $\delta>0$, there exists a partition $\left\{S_{i}\right\}$ of $S$ into open sets such that

$$
\sum_{i} \int_{S_{i}}\left|\nabla_{p} \mathbf{y}-\overline{\mathbf{F}}_{i}\right|^{p} d \mathbf{z}_{p}<\delta \quad \text { with } \overline{\mathbf{F}}_{i}=f_{S_{i}} \nabla_{p} \mathbf{y} d \mathbf{z}_{p} .
$$

Let $\tilde{\mathbf{y}}^{(h)}=\overline{\mathbf{F}}_{i} \mathbf{z}_{p}+\mathbf{y}^{(h)}-\boldsymbol{\omega}^{(h)}$ for $\mathbf{z} \in \Omega_{i}^{1}=S_{i} \times(0,1)$. Clearly $\tilde{\mathbf{y}}^{(h)} \rightarrow \overline{\mathbf{F}}_{i} \mathbf{z}_{p}$ in $W^{1, p}\left(\Omega_{i}^{1}, \mathbb{R}^{3}\right)$ as $h \rightarrow 0$. Using the previous result for piecewise affine functions, we have after summation,

$$
\liminf _{h \rightarrow 0} \sum_{i} e_{1}^{(h)}\left[\tilde{\mathbf{y}}^{(h)} ; \Omega_{i}^{1}\right] \geqq \sum_{i} e_{1}^{(0)}\left[\overline{\mathbf{F}}_{i} \mathbf{z}_{p} ; S_{i}\right] .
$$

Notice that from (4.31), we have

$$
\begin{aligned}
\left|\left\|\kappa \nabla_{p}^{2} \tilde{\mathbf{y}}^{(h)}\right\|_{L^{2}\left(\Omega_{i}^{1}\right)}-\left\|\kappa \nabla_{p}^{2} \mathbf{y}^{(h)}\right\|_{L^{2}\left(\Omega_{i}^{1}\right)}\right| & \leqq\left\|\kappa\left(\nabla_{p}^{2} \tilde{\mathbf{y}}^{(h)}-\nabla_{p}^{2} \mathbf{y}^{(h)}\right)\right\|_{L^{2}\left(\Omega_{i}^{1}\right)} \\
& =\left\|\kappa \nabla_{p}^{2} \boldsymbol{\omega}^{(h)}\right\|_{L^{2}\left(\Omega_{i}^{1}\right)} \rightarrow 0
\end{aligned}
$$

as $h \rightarrow 0$. Using (4.32), (4.34) and the Lipschitz conditions on $\varphi$ and $\bar{\varphi}$, we obtain

$$
\begin{array}{r}
\left|e_{1}^{(h)}\left[\mathbf{y}^{(h)} ; \Omega^{1}\right]-\sum_{i} e_{1}^{(h)}\left[\tilde{\mathbf{y}}^{(h)} ; \Omega_{i}^{1}\right]\right| \leqq C \cdot \delta^{\frac{1}{p}} \\
\left|e_{1}^{(0)}[\mathbf{y}]-\sum_{i} e_{1}^{(0)}\left[\overline{\mathbf{F}}_{i} \mathbf{z}_{p} ; S_{i}\right]\right| \leqq C \cdot \delta^{\frac{1}{p}}
\end{array}
$$

for sufficiently small $h$. Collecting (4.33), (4.35) and (4.36) concludes the proof. 


\section{Film thickness comparable to heterogeneity}

Theorem 3. Suppose $\varphi=\varphi\left(\mathbf{F}, \frac{\mathbf{z}_{p}}{d}, z_{3}\right), \frac{\kappa}{d} \rightarrow \alpha$ and $\frac{h}{d} \rightarrow \beta>0$ as $h \rightarrow 0$. Let $\overline{\mathbf{F}} \in \mathbb{M}^{3 \times 2}$ and $e_{1}^{(h)}$ be defined by (2.6). Then $e_{1}^{(h)} \Gamma$-converges to the functional $e_{1}^{(0)}$ defined by (2.7) if

(i) $\alpha=0$ and

$$
\bar{\varphi}(\overline{\mathbf{F}})=\inf _{k \in \mathbb{N}} \inf _{\boldsymbol{\omega} \in A_{k}^{\beta}} f_{\Omega_{k}^{\beta}} \varphi\left(\overline{\mathbf{F}}+\nabla_{p} \boldsymbol{\omega} \mid \boldsymbol{\omega}_{, 3}, \mathbf{z}_{p}, \frac{z_{3}}{\beta}\right) d \mathbf{z},
$$

where

$$
\begin{aligned}
\Omega_{k}^{\beta} & =k Z \times(0, \beta), \quad Z=(0,1)^{2}, \quad \Sigma^{\beta}=\partial k Z \times(0, \beta), \\
\tilde{A}_{k}^{\beta} & =\left\{\omega: \omega \in W^{1, p}\left(\Omega_{k}^{\beta}, \mathbb{R}^{3}\right),\left.\omega\right|_{\Sigma^{\beta}}=0\right\}
\end{aligned}
$$

(ii) $\alpha>0$ and

$$
\bar{\varphi}(\overline{\mathbf{F}})=\inf _{k \in \mathbb{N}} \inf _{\boldsymbol{\omega} \in A_{k}^{\beta}} f_{\Omega_{k}^{\beta}}\left\{\alpha^{2}\left|\nabla^{2} \boldsymbol{\omega}\right|^{2}+\varphi\left(\overline{\mathbf{F}}+\nabla_{p} \boldsymbol{\omega} \mid \boldsymbol{\omega}_{, 3}, \mathbf{z}_{p}, \frac{z_{3}}{\beta}\right)\right\} d \mathbf{z}
$$

where

$$
A_{k}^{\beta}=\left\{\omega: \omega \in W^{1, p}\left(\Omega_{k}^{\beta}, \mathbb{R}^{3}\right) \cap W^{2,2}\left(\Omega_{k}^{\beta}, \mathbb{R}^{3}\right),\left.\omega\right|_{\Sigma^{\beta}}=\left.\frac{\partial \boldsymbol{\omega}}{\partial n}\right|_{\Sigma^{\beta}}=0\right\} ;
$$

(iii) $\alpha=\infty$ and

$$
\begin{gathered}
\bar{\varphi}(\overline{\mathbf{F}})=Q \tilde{\varphi}_{0}(\overline{\mathbf{F}}), \\
\tilde{\varphi}_{0}(\overline{\mathbf{F}})=\inf _{\mathbf{b} \in \mathbb{R}^{3}} \tilde{\varphi}(\overline{\mathbf{F}} \mid \mathbf{b}), \tilde{\varphi}(\mathbf{F})=\int_{Z \times(0,1)} \varphi(\mathbf{F}, \mathbf{z}) d \mathbf{z}
\end{gathered}
$$

where $Q \tilde{\varphi}_{0}$ is the lower quasi-convex envelope of $\tilde{\varphi}_{0}$.

The proof of Theorem 3 for finite $\alpha \geqq 0$ is very similar to that of Theorem 2 . If $\frac{h}{d}=\beta$, we construct a recovery sequence for an affine function $\mathbf{y}=\overline{\mathbf{F}} \mathbf{z}_{p}$ using the scaling

$$
\mathbf{y}^{(h)}=\overline{\mathbf{F}} \mathbf{z}_{p}+d \omega\left(\frac{\mathbf{z}_{p}}{d}, \frac{h z_{3}}{d}\right)
$$

where $\omega(\mathbf{z}) \in A_{k}^{\beta}$. The proof of lower bound also follows exactly that of Theorem 2 by using the same scaling (5.6). The case $\alpha=\infty$ is a corollary of Theorem 1(ii) since $\frac{\kappa}{h}=\frac{\kappa}{d} \frac{d}{h} \rightarrow \infty$ as $h \rightarrow 0$ in this case.

We have a similar theorem when the in-plane heterogeneity vanishes.

Theorem 4. Suppose $\varphi=\varphi\left(\mathbf{F}, z_{3}\right)$ and $\frac{\kappa}{h} \rightarrow \alpha^{\prime}$ as $h \rightarrow 0$. Let $\overline{\mathbf{F}} \in \mathbb{M}^{3 \times 2}$ and $e_{1}^{(h)}$ be defined by (2.6). Then $e_{1}^{(h)} \Gamma$-converges to the functional $e_{1}^{(0)}$ defined by (2.7) if 
(i) $\alpha^{\prime}=0$ and

$$
\bar{\varphi}(\overline{\mathbf{F}})=\inf _{k \in \mathbb{N}} \inf _{\boldsymbol{\omega} \in \tilde{A}_{k}^{1}} f_{\Omega_{k}^{1}} \varphi\left(\overline{\mathbf{F}}+\nabla_{p} \boldsymbol{\omega} \mid \boldsymbol{\omega}_{, 3}, z_{3}\right) d \mathbf{z}
$$

where $\Omega_{k}^{1}$ and $\tilde{A}_{k}^{1}$ are defined by (5.2) and (5.3);

(ii) $\alpha^{\prime}>0$ and

$$
\bar{\varphi}(\overline{\mathbf{F}})=\inf _{k \in \mathbb{N}} \inf _{\boldsymbol{\omega} \in A_{k}^{1}} f_{\Omega_{k}^{1}}\left\{\alpha^{\prime 2}\left|\nabla^{2} \boldsymbol{\omega}\right|^{2}+\varphi\left(\overline{\mathbf{F}}+\nabla_{p} \boldsymbol{\omega} \mid \boldsymbol{\omega}_{, 3}, z_{3}\right)\right\} d \mathbf{z}
$$

where $\Omega_{k}^{1}$ and $A_{k}^{1}$ are defined by (5.2) and (5.5);

(iii) $\alpha^{\prime}=\infty$ and

$$
\begin{gathered}
\bar{\varphi}(\overline{\mathbf{F}})=Q \tilde{\varphi}_{0}(\overline{\mathbf{F}}), \\
\tilde{\varphi}_{0}(\overline{\mathbf{F}})=\inf _{\mathbf{b} \in \mathbb{R}^{3}} \tilde{\varphi}(\overline{\mathbf{F}} \mid \mathbf{b}), \quad \tilde{\varphi}(\mathbf{F})=\int_{0}^{1} \varphi\left(\mathbf{F}, z_{3}\right) d z_{3},
\end{gathered}
$$

where $Q \tilde{\varphi}_{0}$ is the lower quasi-convex envelope of $\tilde{\varphi}_{0}$.

Remark 5. If our film is homogeneous and the interfacial energy is negligible ( $\alpha=\alpha^{\prime}=0$ ), all our results coincide with that of LE DRET \& RAOult [21], i.e., $\bar{\varphi}(\overline{\mathbf{F}})=Q \varphi_{0}(\overline{\mathbf{F}})$. This is obvious in Theorem 1 and Theorem 2, but not in Theorem 3 and Theorem 4. So we explain this in some detail. Consider a homogeneous film with energy density $\varphi=\varphi(\mathbf{F})$ and let

$$
\varphi_{0}(\overline{\mathbf{F}})=\inf _{\mathbf{b} \in \mathbb{R}^{3}} \varphi(\overline{\mathbf{F}} \mid \mathbf{b}) .
$$

Assume $\varphi$ satisfies (2.4) and (2.5). A similar argument used in Remark 2 shows that $\varphi_{0}$ is well defined and the infimum is achieved. Further, $\varphi_{0}$ also enjoys the growth and coercivity estimates (2.4). Hence, $W^{1, p}$ quasi-convexification is equal to $W^{1, \infty}$ quasi-convexification and $Q \varphi_{0}$ can be expressed as

$$
Q \varphi_{0}(\overline{\mathbf{F}})=\inf _{\hat{\boldsymbol{\omega}} \in W_{0}^{1, p}(Z)} f_{Z} \varphi_{0}\left(\overline{\mathbf{F}}+\nabla_{p} \hat{\boldsymbol{\omega}}\right) d \mathbf{z}_{p},
$$

where $Z=(0,1)^{2}$. On the other hand, for homogeneous films, $\bar{\varphi}$ defined in (5.1) becomes

$$
\bar{\varphi}(\overline{\mathbf{F}})=\inf _{k \in \mathbb{N}} \inf _{\boldsymbol{\omega} \in \tilde{A}_{k}^{\beta}} f_{\Omega_{k}^{\beta}} \varphi\left(\overline{\mathbf{F}}+\nabla_{p} \boldsymbol{\omega} \mid \boldsymbol{\omega}, 3\right) d \mathbf{z}
$$

for any finite $\beta>0$. We wish to show $\bar{\varphi}=Q \varphi_{0}$.

First, it is clear that

$$
\bar{\varphi}(\overline{\mathbf{F}}) \geqq Q \varphi_{0}(\overline{\mathbf{F}}) .
$$

To prove the reverse inequality, notice that there exist sequences of $\hat{\boldsymbol{\omega}}^{\delta} \in W_{0}^{1, p}$ $\left(Z, \mathbb{R}^{3}\right)$ and $\mathbf{b}^{\delta} \in L^{p}\left(Z, \mathbb{R}^{3}\right)$ such that

$$
f_{Z} \varphi_{0}\left(\overline{\mathbf{F}}+\nabla_{p} \hat{\boldsymbol{\omega}}^{\delta}\right) d \mathbf{z}_{p}=f_{Z} \varphi\left(\overline{\mathbf{F}}+\nabla_{p} \hat{\boldsymbol{\omega}}^{\delta} \mid \mathbf{b}^{\delta}\right) d \mathbf{z}_{p} \rightarrow Q \varphi_{0}(\overline{\mathbf{F}})
$$


as $\delta \rightarrow 0$. Since $C_{0}^{\infty}\left(Z, \mathbb{R}^{3}\right)$ is dense in $L^{p}\left(Z, \mathbb{R}^{3}\right)$, we may assume $\mathbf{b}^{\delta} \in$ $C_{0}^{\infty}\left(Z, \mathbb{R}^{3}\right)$ (see Remark 3). Let $k \in \mathbb{N}$ and define

$$
\mathbf{y}^{(k, \delta)}=\overline{\mathbf{F}} \mathbf{z}_{p}+k \hat{\boldsymbol{\omega}}^{\delta}\left(\frac{\mathbf{z}_{p}}{k}\right)+\mathbf{b}^{\delta}\left(\frac{\mathbf{z}_{p}}{k}\right) z_{3} .
$$

It is clear that $\left(\mathbf{y}^{(k, \delta)}-\overline{\mathbf{F}} \mathbf{z}_{p}\right) \in \tilde{A}_{k}^{\beta}$ defined by (5.3). Therefore,

$$
\bar{\varphi}(\overline{\mathbf{F}}) \leqq f_{\Omega_{k}^{\beta}} \varphi\left(\nabla \mathbf{y}^{(k, \delta)}\right) d \mathbf{z}
$$

for all $k \in \mathbb{N}$ and $\delta>0$. Notice that by changing variable $\hat{\mathbf{z}}_{p}=\frac{\mathbf{z}_{p}}{k}$ and $\hat{z}_{3}=z_{3}$, (5.13) becomes

$$
\bar{\varphi}(\overline{\mathbf{F}}) \leqq \frac{1}{\beta} \int_{0}^{\beta} \int_{Z} \varphi\left(\overline{\mathbf{F}}+\nabla_{p} \hat{\boldsymbol{\omega}}^{\delta}\left(\hat{\mathbf{z}}_{p}\right)+\frac{1}{k} \nabla_{p} \mathbf{b}^{\delta}\left(\hat{\mathbf{z}}_{p}\right) \hat{z}_{3} \mid \mathbf{b}^{\delta}\left(\hat{\mathbf{z}}_{p}\right)\right) d \hat{\mathbf{z}}_{p} d \hat{z}_{3} .
$$

Let $k \rightarrow \infty, \delta \rightarrow 0$ in (5.14) and recall (5.11). We have

$$
\bar{\varphi}(\overline{\mathbf{F}}) \leqq Q \varphi_{0}(\overline{\mathbf{F}}) .
$$

Remark 6. If the film does not contain any out-of-plane heterogeneity, i.e., $\varphi=$ $\varphi\left(\mathbf{F}, \frac{\mathbf{z}_{p}}{d}\right)$, then Theorem 1(i) and Theorem 2(i) imply that the ratio $\frac{\kappa}{h}$ is irrelevant to the effective energy. In particular, the effective energy of a homogeneous film is independent of the ratio $\frac{\kappa}{h}$. This ratio $\frac{\kappa}{h}$ is important only if the film contains outof-plane heterogeneity such as discussed in Theorem 4 . We provide an example to explain this. Let $S=(0, L), \Omega^{1}=S \times(0,1)$, and $\mathbf{y}: \Omega^{1} \rightarrow \mathbb{R}^{2}$ be the deformation. The energy per unit thickness for this homogeneous thin film is

$$
e_{1}^{(h)}[\mathbf{y}]=\int_{\Omega^{1}}\left\{\kappa^{2}\left(\left|\mathbf{y}_{, 11}\right|^{2}+\frac{2}{h^{2}}\left|\mathbf{y}_{, 12}\right|^{2}+\frac{1}{h^{4}}\left|\mathbf{y}_{, 22}\right|^{2}\right)+\varphi\left(\mathbf{y}_{, 1} \mid \frac{1}{h} \mathbf{y}_{, 2}\right)\right\} d \mathbf{z}
$$

and the effective energy as $h \rightarrow 0$ is

$$
e_{1}^{(0)}[\mathbf{y}]=\int_{S} Q \varphi_{0}\left(\frac{\partial \mathbf{y}}{\partial z_{1}}\right) d z_{1} .
$$

Notice that the effective energy density $\bar{\varphi}$ is independent of the ratio $\frac{\kappa}{h}$. Let us now explain this using the following example. Consider the material with the local energy density $\varphi^{(1)}(\mathbf{F}): \mathbb{M}^{2 \times 2} \rightarrow \mathbb{R}$ of the form

$$
\varphi^{(1)}(\mathbf{F})=\frac{1}{2}\left\{\left(F_{11}-1\right)^{2}+\left(F_{21}^{2}-1\right)^{2}+F_{12}^{2}+\left(F_{22}-1\right)^{2}\right\} .
$$

The minimizers of $\varphi^{(1)}$ are

$$
\mathbf{F}_{1}^{(1)}=\left(\begin{array}{ll}
1 & 0 \\
1 & 1
\end{array}\right), \quad \mathbf{F}_{2}^{(1)}=\left(\begin{array}{cc}
1 & 0 \\
-1 & 1
\end{array}\right) .
$$

Suppose the edge of this thin film is clamped. We now show that the total energy $e_{1}^{(h)}$ tends to zero as $h$ tends to zero irrespective of the ratio $\frac{\kappa}{h}$. First consider the 


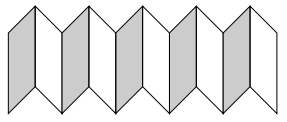

(a)

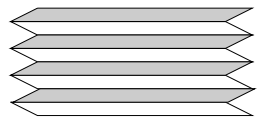

(b)

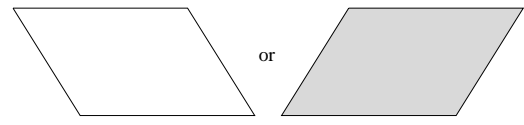

(c)

Fig. 2. A heterogeneous thin film with clamped edges. (a) The sequence that minimizes the energy with density $\varphi^{(1)}$ for $\alpha^{\prime} \geqq 0$. Here the darkly shaded and white regions represent $\mathbf{F}_{1}^{(1)}$ and $\mathbf{F}_{2}^{(1)}$ defined by (5.16). (b) The sequence that minimizes the energy with density $\varphi^{(2)}$ only for $\alpha^{\prime}=0$. Here the darkly shaded and white regions represent $\mathbf{F}_{1}^{(2)}$ and $\mathbf{F}_{2}^{(2)}$ defined by (5.18). (c) The sequence that minimizes the energy with density $\varphi^{(2)}$ for $\alpha^{\prime} \geqq 0$. Here the darkly shaded and white regions represent $\mathbf{F}_{1}^{(2)}$ and $\mathbf{F}_{2}^{(2)}$ defined by (5.18).

case $\kappa=0$. Then, at each $h>0$, the sequence shown schematically in Fig. 2(a) is clearly a minimizing sequence and it follows that

$$
e_{1}^{(h)}\left[\mathbf{y}^{(h)}\right] \rightarrow 0 \quad \text { as } h \rightarrow 0 .
$$

If $\kappa \neq 0$ but is small, i.e., $\alpha^{\prime}=\frac{\kappa}{h} \stackrel{h}{\rightarrow} 0$, a slight refinement establishes the same result. On the other hand, if $\alpha^{\prime}>0$, it is not clear whether Fig. 2(a) minimizes $e_{1}^{(h)}$ as it contains too much interfacial energy. Now set

$$
f\left(z_{1}\right)= \begin{cases}z_{1} & \text { for } 0 \leqq z_{1} \leqq \frac{1}{2} \\ -z_{1}+\frac{1}{2} & \text { for } \frac{1}{2} \leqq z_{1} \leqq 1\end{cases}
$$

Let $\chi^{(m)} \in C_{0}^{\infty}((0,1))$ converges strongly to $f$ in $W_{0}^{1,4}((0,1))$ as $m \rightarrow \infty$. For each fixed $m$, extend $\chi^{(m)}$ periodically to $\mathbb{R}$ and consider

$$
y_{1}^{(1, h, m)}=z_{1}, \quad y_{2}^{(1, h, m)}=d L \chi^{(m)}\left(\frac{z_{1}}{d L}\right)+h z_{2},
$$

where $1 / d(h)$ is chosen to be the largest integer such that $1 / d(h) \leqq 1 / \sqrt{\kappa(h)}$. Substituting (5.17) into (5.15), we find that the energy is driven to zero if $h \rightarrow 0$ first and $m \rightarrow \infty$ next. By standard diagonalization process, we can find a subsequence $\mathbf{y}^{(h)}$ such that $e_{1}^{(h)}\left[\mathbf{y}^{(h)}\right] \rightarrow 0$ as $h \rightarrow 0$.

Next consider another material with the local energy density $\varphi^{(2)}(\mathbf{F}): \mathbb{M}^{2 \times 2} \rightarrow$ $\mathbb{R}$ of the form

$$
\varphi^{(2)}(\mathbf{F})=\frac{1}{2}\left\{\left(F_{11}-1\right)^{2}+F_{21}^{2}+\left(F_{12}^{2}-1\right)^{2}+\left(F_{22}-1\right)^{2}\right\} .
$$

The minimizers for $\varphi^{(2)}$ are

$$
\mathbf{F}_{1}^{(2)}=\left(\begin{array}{ll}
1 & 1 \\
0 & 1
\end{array}\right), \quad \mathbf{F}_{2}^{(2)}=\left(\begin{array}{cc}
1 & -1 \\
0 & 1
\end{array}\right) .
$$

Now if the edge of the film is clamped and if $\kappa=0$ or $\alpha^{\prime}=0$, Fig. 2(b) is clearly one sequence minimizing the energy of this strip. On the other hand, if $\alpha^{\prime}>0$, it can be shown that the sequence in Fig. 2(b) does not minimize the energy. Instead, the 
sequence that minimizes the energy in this case looks like that in Fig. 2(c). Indeed, set $g\left(z_{1}\right)=1$ and let $\eta^{(m)} \in C_{0}^{\infty}((0, L))$ converge strongly to $g$ in $L^{4}((0, L))$. Consider

$$
y_{1}^{(2, h, m)}=z_{1} \pm h \eta^{(m)}\left(z_{1}\right) z_{2}, \quad y_{2}^{(2, h, m)}=h z_{2} .
$$

Using an argument similar to the above, we can find a subsequence $\mathbf{y}^{(h)}$ such that $e_{1}^{(h)}\left[\mathbf{y}^{(h)}\right] \rightarrow 0$ as $h \rightarrow 0$.

In summary, material can form microstructure freely with interfaces which are perpendicular to the film (Fig. 2(a)) and such interfaces cost vanishing energy as $h \rightarrow 0$ independent of $\frac{\kappa}{h}$. Further, material does not need to form the out-of-plane fine-scale microstructure to reduce the energy (Fig. 2(c)) as long as there is no out-of-plane heterogeneity contained in the film.

\section{Film thickness much larger than heterogeneity}

Let us consider the case $\kappa=0, \varphi=\varphi\left(\mathbf{F}, \frac{\mathbf{z}_{p}}{d}\right)$ and the following change of variables

$$
\hat{\mathbf{z}}_{p}=\frac{\mathbf{z}_{p}}{h}, \quad \hat{z}_{3}=z_{3}, \quad h \hat{\mathbf{y}}\left(\hat{\mathbf{z}}_{p}, \hat{z}_{3}\right)=\mathbf{y}\left(h \hat{\mathbf{z}}_{p}, \hat{z}_{3}\right) .
$$

Then, $e_{1}^{(h)}$ becomes

$$
h^{2} \int_{0}^{1} \int_{\frac{S}{h}} \varphi\left(\nabla \hat{\mathbf{y}}, \frac{\hat{\mathbf{z}}_{p}}{\frac{d}{h}}\right) d \hat{\mathbf{z}}_{p} d \hat{z}_{3} .
$$

Since $\frac{h}{d} \rightarrow \infty$ as $h \rightarrow 0$, (6.2) suggests that $\bar{\varphi}$ is obtained by homogenizing bulk heterogeneous material first, and then passing to the thin-film limit. This is precisely phrased by the following theorem.

Theorem 5. Suppose $\varphi=\varphi\left(\mathbf{F}, \frac{\mathbf{z}_{p}}{d}\right), \kappa=0$ and $\frac{h}{d} \rightarrow \infty$ as $h \rightarrow 0$. Then, $e_{1}^{(h)}$ $\Gamma$-converges to the functional $e_{1}^{(0)}$ defined by (2.7) if

$$
\begin{aligned}
\bar{\varphi}(\overline{\mathbf{F}}) & =Q \varphi_{0}^{H}(\overline{\mathbf{F}}), \\
\varphi_{0}^{H}(\overline{\mathbf{F}}) & =\inf _{\mathbf{b} \in \mathbb{R}^{3}} \varphi^{H}(\overline{\mathbf{F}} \mid \mathbf{b}), \\
\varphi^{H}(\mathbf{F}) & =\inf _{k \in \mathbb{N}} \inf _{\boldsymbol{\omega} \in W_{0}^{1, p}(k \hat{Z})} f_{k \hat{Z}} \varphi\left(\mathbf{F}+\nabla \boldsymbol{\omega}, \mathbf{z}_{p}\right) d \mathbf{z},
\end{aligned}
$$

where $Q \varphi_{0}^{H}(\overline{\mathbf{F}})$ is the lower quasi-convex envelope of $\varphi_{0}^{H}, \hat{Z}=(0,1)^{3}$ and $\mathbf{F}=$ $(\overline{\mathbf{F}} \mid \mathbf{b}) \in \mathbb{M}^{3 \times 3}$. 
The establishment of the lower bound [12] will be based on the following two lemmas.

Lemma 2. Suppose $\mathbf{y}^{(h)} \rightarrow \mathbf{y}$ in $W^{1, p}\left(\Omega^{1}, \mathbb{R}^{3}\right)$ as $h \rightarrow 0$ and assume $\liminf _{h \rightarrow 0} e_{1}^{(h)}\left[\mathbf{y}^{(h)}\right]$ is finite. Then, there exists a subsequence $\mathbf{y}^{(h)}$ (not relabeled), and a sequence $\tilde{\mathbf{y}}^{(h)}$ such that

$$
\left|E_{h}\right|=\left|\left\{\mathbf{y}^{(h)} \neq \tilde{\mathbf{y}}^{(h)}\right\} \cup\left\{\nabla \mathbf{y}^{(h)} \neq \nabla \tilde{\mathbf{y}}^{(h)}\right\}\right| \rightarrow 0
$$

as $h \rightarrow 0,\left(\left|\nabla_{p} \tilde{\mathbf{y}}^{(h)}\right|^{p}\right)$ and $\left(\left|\frac{1}{h} \tilde{\mathbf{y}}_{, 3}^{(h)}\right|^{p}\right)$ are equi-integrable, and

$$
\liminf _{h \rightarrow 0} e_{1}^{(h)}\left[\tilde{\mathbf{y}}^{(h)}\right] \leqq \liminf _{h \rightarrow 0} e_{1}^{(h)}\left[\mathbf{y}^{(h)}\right]
$$

Proof. Due to the coercivity (2.4) on $\varphi$, it is clear that

$$
\sup _{h} \int_{\Omega^{1}}\left(\left|\nabla_{p} \mathbf{y}^{(h)}\right|^{p}+\left|\frac{1}{h} \mathbf{y}_{, 3}^{(h)}\right|^{p}\right)<+\infty .
$$

By passing to a subsequence $\mathbf{y}^{(h)}$ (not relabeled), we may assume this subsequence achieves the $\liminf _{h \rightarrow 0} e_{1}^{(h)}\left[\mathbf{y}^{(h)}\right]$. We extend the definition of $\mathbf{y}^{(h)}$ to $S \times \mathbb{R}$, first defining $\mathbf{y}^{(h)}$ on $S \times(0,2)$ by $\mathbf{y}^{(h)}\left(\mathbf{z}_{p}, z_{3}\right)=\mathbf{y}^{(h)}\left(\mathbf{z}_{p}, 2-z_{3}\right)$, and then by 2 periodicity in the $z_{3}$-direction.

We then define

$$
\boldsymbol{\omega}^{(h)}\left(\mathbf{z}_{p}, z_{3}\right)=\mathbf{y}^{(h)}\left(\mathbf{z}_{p}, \frac{1}{h} z_{3}\right) .
$$

We have

$$
\begin{aligned}
\int_{\Omega^{1}}\left|\nabla_{p} \omega^{(h)}\right|^{p} d \mathbf{z} & =h \int_{S \times\left(0, \frac{1}{h}\right)}\left|\nabla_{p} \mathbf{y}^{(h)}\right|^{p} d \mathbf{z} \leqq h\left[\left[\frac{1}{h}+1\right]\right] \int_{\Omega^{1}}\left|\nabla_{p} \mathbf{y}^{(h)}\right|^{p} d \mathbf{z}, \\
\int_{\Omega^{1}}\left|\omega_{, 3}^{(h)}\right|^{p} d \mathbf{z} & =h \int_{S \times\left(0, \frac{1}{h}\right)}\left|\frac{1}{h} \mathbf{y}_{, 3}^{(h)}\right|^{p} d \mathbf{z} \leqq h\left[\left[\frac{1}{h}+1\right]\right] \int_{\Omega^{1}}\left|\frac{1}{h} \mathbf{y}_{, 3}^{(h)}\right|^{p} d \mathbf{z},
\end{aligned}
$$

where $[[x]]$ denotes the largest integer which is smaller than $x$. Using the Decomposition Lemma 1.2 in [23], we can find a subsequence $\omega^{(h)}$ (not relabeled), and a sequence of functions $\mathbf{u}^{(h)}$ in $W^{1, p}\left(\Omega^{1}, \mathbb{R}^{3}\right)$, such that

$$
\lim _{h \rightarrow 0}\left|\left\{\mathbf{u}^{(h)} \neq \omega^{(h)}\right\} \cup\left\{\nabla \mathbf{u}^{(h)} \neq \nabla \boldsymbol{\omega}^{(h)}\right\}\right|=0,
$$

and $\left(\left|\nabla \mathbf{u}^{(h)}\right|^{p}\right)$ is equi-integrable. Moreover, following the construction in the proof of Lemma 1.2 in [23], we see that the functions $\mathbf{u}^{(h)}$ can be chosen to be $2 h$-periodic in the $z_{3}$-direction.

Now let

$$
\tilde{\mathbf{y}}^{(h)}\left(\mathbf{z}_{p}, z_{3}\right)=\mathbf{u}^{(h)}\left(\mathbf{z}_{p}, h z_{3}\right) .
$$

If $A \subset \Omega^{1}, A_{h}=\left\{\left(\mathbf{z}_{p}, h z_{3}\right): \mathbf{z} \in A\right\}$ and

$$
A^{\prime}=\bigcup\left\{A_{h}+2 k h \mathbf{e}_{3}: k \in \mathbb{Z},\left(A_{h}+2 k h \mathbf{e}_{3}\right) \cap \Omega^{1} \neq \emptyset\right\},
$$


then we have

$$
\begin{aligned}
\int_{A}\left|\nabla_{p} \tilde{\mathbf{y}}^{(h)}\right|^{p} d \mathbf{z} & =\frac{1}{h} \int_{A_{h}}\left|\nabla_{p} \mathbf{u}^{(h)}\right|^{p} d \mathbf{z} \leqq 2 \int_{A^{\prime}}\left|\nabla_{p} \mathbf{u}^{(h)}\right|^{p} d \mathbf{z}, \\
\int_{A}\left|\frac{1}{h} \tilde{\mathbf{y}}_{, 3}\right|^{p} d \mathbf{z} & =\frac{1}{h} \int_{A_{h}}\left|\mathbf{u}_{, 3}^{(h)}\right|^{p} d \mathbf{z} \leqq 2 \int_{A^{\prime}}\left|\mathbf{u}_{, 3}^{(h)}\right|^{p} d \mathbf{z} .
\end{aligned}
$$

Since we have $\left|A^{\prime}\right| \leqq c|A|$, these inequalities show that $\left(\left|\nabla_{p} \tilde{\mathbf{y}}^{(h)}\right|^{p}\right)$ and $\left(\left|\frac{1}{h} \tilde{\mathbf{y}}_{, 3}\right|^{p}\right)$ are equi-integrable.

Let

$$
E_{h}=\left\{\mathbf{y}^{(h)} \neq \tilde{\mathbf{y}}^{(h)}\right\} \cup\left\{\nabla \mathbf{y}^{(h)} \neq \nabla \tilde{\mathbf{y}}^{(h)}\right\} .
$$

We have then $\left|E_{h}\right| \rightarrow 0$ as $h \rightarrow 0$ and

$$
\int_{E_{h}} \varphi\left(\nabla_{p} \tilde{\mathbf{y}}^{(h)} \mid \frac{1}{h} \tilde{\mathbf{y}}_{, 3}^{(h)}, \frac{\mathbf{z}_{p}}{d}\right) d \mathbf{z} \leqq c_{2} \int_{E_{h}}\left(1+\left|\nabla_{p} \tilde{\mathbf{y}}^{(h)}\right|^{p}+\left|\frac{1}{h} \tilde{\mathbf{y}}_{, 3}^{(h)}\right|^{p}\right) d \mathbf{z} \rightarrow 0
$$

as $h \rightarrow 0$. Therefore, we have

$$
\begin{aligned}
& \liminf _{h \rightarrow 0} e_{1}^{(h)}\left[\tilde{\mathbf{y}}^{(h)}\right] \\
= & \liminf _{h \rightarrow 0}\left(\int_{\Omega^{1} \backslash E_{h}} \varphi\left(\nabla_{p} \tilde{\mathbf{y}}^{(h)} \mid \frac{1}{h} \tilde{\mathbf{y}}_{, 3}^{(h)}, \frac{\mathbf{z}_{p}}{d}\right) d \mathbf{z}+\int_{E_{h}} \varphi\left(\nabla_{p} \tilde{\mathbf{y}}^{(h)} \mid \frac{1}{h} \tilde{\mathbf{y}}_{, 3}^{(h)}, \frac{\mathbf{z}_{p}}{d}\right) d \mathbf{z}\right) \\
= & \liminf _{h \rightarrow 0} \int_{\Omega^{1} \backslash E_{h}} \varphi\left(\nabla_{p} \mathbf{y}^{(h)} \mid \frac{1}{h} \mathbf{y}_{, 3}^{(h)}, \frac{\mathbf{z}_{p}}{d}\right) d \mathbf{z} \leqq \liminf _{h \rightarrow 0} e_{1}^{(h)}\left[\mathbf{y}^{(h)}\right]
\end{aligned}
$$

by the equi-integrability above. This concludes the proof.

The next lemma is suggested by MüLLER [35] (see also [14], Chapter 22).

Lemma 3. Let $S \subset \mathbb{R}^{2}$ be a Lipschitz set and $\Omega^{1}=S \times(0,1)$. For all $M, \delta>0$ there exists $\varepsilon_{0}>0$ such that for all $\varepsilon<\varepsilon_{0}$ and $\mathbf{u} \in W^{1, p}\left(\Omega^{1}, \mathbb{R}^{3}\right)$ with $\|\nabla \mathbf{u}\|_{\mathrm{L}^{p}} \leqq$ $M$ there exists $\mathbf{v} \in W^{1, p}\left(\Omega^{1}, \mathbb{R}^{3}\right)$ such that

$$
\int_{\Omega^{1}} \varphi\left(\nabla \mathbf{u}, \frac{\mathbf{z}_{p}}{\varepsilon}\right) d \mathbf{z} \geqq \int_{\Omega^{1}} \varphi^{H}(\nabla \mathbf{u}+\nabla \mathbf{v}) d \mathbf{z}-\delta,
$$

and $\|\mathbf{v}\|_{\mathrm{L}^{p}} \leqq \delta$. The choice of $\varepsilon_{0}$ can be uniform for all $S^{\prime}$ translations of $S$.

Proof. The proof is by contradiction. Suppose that there exist $M, \delta>0$, a sequence $\left(\varepsilon_{j}\right)$ of positive numbers converging to 0 , and a sequence $\left(\mathbf{u}_{j}\right)$ in $W^{1, p}\left(\Omega^{1}, \mathbb{R}^{3}\right)$ with $\left\|\nabla \mathbf{u}_{j}\right\|_{\mathrm{L}^{p}} \leqq M$ such that

$$
\int_{\Omega^{1}} \varphi\left(\nabla \mathbf{u}_{j}, \frac{\mathbf{z}_{p}}{\varepsilon_{j}}\right) d \mathbf{z}<\inf \left\{\int_{\Omega^{1}} \varphi^{H}\left(\nabla \mathbf{u}_{j}+\nabla \mathbf{v}\right) d \mathbf{z}:\|\mathbf{v}\|_{L^{p}} \leqq \delta\right\}-\delta .
$$

By passing to a subsequence (not relabeled), we can suppose that $\left(\mathbf{u}_{j}\right)$ converges weakly in $W^{1, p}\left(\Omega^{1}, \mathbb{R}^{3}\right)$ to a function $\mathbf{u}$, and that

$$
\left\|\mathbf{u}_{j}-\mathbf{u}\right\|_{\mathrm{L}^{p}} \leqq \frac{\delta}{2}
$$


for all $j$. Note that the last inequality implies that

$$
\begin{aligned}
\inf \left\{\int_{\Omega^{1}} \varphi^{H}\left(\nabla \mathbf{u}_{j}+\nabla \mathbf{v}\right) d \mathbf{z}:\|\mathbf{v}\|_{\mathrm{L}^{p}} \leqq \delta\right\} \\
\quad \leqq \inf \left\{\int_{\Omega^{1}} \varphi^{H}(\nabla \mathbf{w}) d x:\|\mathbf{w}-\mathbf{u}\|_{\mathrm{L}^{p}} \leqq t\right\}
\end{aligned}
$$

for all $t \leqq \delta / 2$. Hence, by (6.8) and by the lim inf inequality of $\Gamma$-convergence, we get

$$
\begin{aligned}
\int_{\Omega^{1}} \varphi^{H}(\nabla \mathbf{u}) d \mathbf{z} & \leqq \liminf _{j} \int_{\Omega^{1}} \varphi\left(\nabla \mathbf{u}_{j}, \frac{\mathbf{z}_{p}}{\varepsilon_{j}}\right) d \mathbf{z} \\
& <\operatorname{supinf}_{t>0}\left\{\int_{\Omega^{1}} \varphi^{H}(\nabla \mathbf{w}) d \mathbf{z}:\|\mathbf{w}-\mathbf{u}\|_{\mathrm{L}^{p}} \leqq t\right\}-\delta \\
& =\liminf _{\mathbf{w} \rightarrow \mathbf{u}} \int_{\Omega^{1}} \varphi^{H}(\nabla \mathbf{w}) d \mathbf{z}-\delta \\
& =\int_{\Omega^{1}} \varphi^{H}(\nabla \mathbf{u}) d \mathbf{z}-\delta,
\end{aligned}
$$

which is a contradiction.

It is clear that by the translation-invariance argument in the $\mathbf{z}_{p}$ plane, it suffices to consider all the sets $\mathbf{z}_{p}+S$ with $\mathbf{z}_{p} \in[0,1]^{2}$ in order to prove that the thesis of the theorem holds uniformly. In this case, the proof above still works with an additional compactness argument for $\mathbf{z}_{p}$.

Proof of Theorem 5. We start with the lower bound. It suffices to consider the case that $\mathbf{y}=\overline{\mathbf{F}} \mathbf{z}_{p}$. The extension to arbitrary function follows Part B of the proof of Theorem 2. Let $\mathbf{y}^{(h)} \rightarrow \overline{\mathbf{F}} \mathbf{z}_{p}$ in $W^{1, p}\left(\Omega^{1}, \mathbb{R}^{3}\right)$ as $h \rightarrow 0$ and assume $\liminf _{h \rightarrow 0} e_{1}^{(h)}\left[\mathbf{y}^{(h)}\right]$ is finite; else the result follows. We further assume $S=$ $(0,1)^{2}$; it will be clear that the proof is insensitive to this assumption.

Set $\boldsymbol{\omega}^{(h)}=\mathbf{y}^{(h)}-\overline{\mathbf{F}} \mathbf{z}_{p}$. Using a cut-off argument near $\partial(0,1)^{2}$ (or the so-called fundamental estimate in Chapter 24 of [14]), we can suppose that we have $\omega^{(h)}=0$ if $\operatorname{dist}\left(\mathbf{z}_{p}, \partial(0,1)^{2}\right) \leqq 2 h$, and, by Lemma 2, we can also suppose that the sequence $\left(\left|\left(\nabla_{p} \omega^{(h)} \mid \frac{1}{h} \omega_{, 3}^{(h)}\right)\right|^{p}\right)$ is equi-integrable.

We set

Note that

$$
\mathbf{u}^{(h)}(\mathbf{z})=\frac{1}{h} \omega^{(h)}\left(h \mathbf{z}_{p}, z_{3}\right), \quad T_{h}=\frac{1}{h}, \quad \mathbf{F}=(\overline{\mathbf{F}}, 0) .
$$

$$
e_{1}^{(h)}\left[\mathbf{y}^{(h)}\right]=\frac{1}{T_{h}^{2}} \int_{\left(0, T_{h}\right)^{2} \times(0,1)} \varphi\left(\mathbf{F}+\nabla \mathbf{u}^{(h)}, \frac{\mathbf{z}_{p}}{\frac{d}{h}}\right) d \mathbf{z},
$$

and that $\mathbf{u}^{(h)}=0$ if $\operatorname{dist}\left(\mathbf{z}_{p}, \partial\left(0, T_{h}\right)^{2}\right) \leqq 2$.

Define

$$
I_{h}=\left\{i \in \mathbb{Z}^{2}: 1 \leqq i_{1}, i_{2} \leqq T_{h}-2\right\}, \quad Z=(0,1)^{2}, \quad \Omega_{i}=(i+Z) \times(0,1) .
$$

Note that

$$
\bigcup_{i \in I_{h}}(i+Z)=\left(1,\left[\left[T_{h}-1\right]\right]\right)^{2},
$$


so that $\mathbf{u}^{(h)}=0$ on

$$
C_{h}:=\left(\left(0, T_{h}\right)^{2} \times(0,1)\right) \backslash \bigcup_{i \in I_{h}} \Omega_{i}
$$

We can write

$$
\begin{aligned}
\int_{\left(0, T_{h}\right)^{2} \times(0,1)} \varphi\left(F+\nabla \mathbf{u}^{(h)}, \frac{\mathbf{z}_{p}}{\frac{d}{h}}\right) d \mathbf{z}= & \sum_{i \in I_{h}} \int_{\Omega_{i}} \varphi\left(F+\nabla \mathbf{u}^{(h)}, \frac{\mathbf{z}_{p}}{\frac{d}{h}}\right), d \mathbf{z} \\
& +\int_{C_{h}} \varphi\left(F, \frac{\mathbf{z}_{p}}{\frac{d}{h}}\right) d \mathbf{z .}
\end{aligned}
$$

Let $M>0$ be fixed, and let

$$
I_{h}^{1}=\left\{i \in I_{h}:\left\|\nabla \mathbf{u}^{(h)}\right\|_{\mathrm{L}^{p}} \leqq M\right\}, \quad I_{h}^{2}=I_{h} \backslash I_{h}^{1} .
$$

Note that since

$$
\int_{\left(0, T_{h}\right)^{2} \times(0,1)}\left|\nabla \mathbf{u}^{(h)}\right|^{p} d \mathbf{z} \leqq c T_{h}^{2}
$$

for some constant $c$ (independent of $h$ ), we have

$$
\#\left(I_{h}^{2}\right) \leqq o(1) T_{h}^{2} \quad \text { as } M \rightarrow+\infty .
$$

For all $i \in I_{h}^{1}$ we can apply Lemma 3, and find that for $h$ small enough (independent of $i$ ) there exist functions $\mathbf{v}_{h}^{i} \in W^{1, p}\left(\Omega_{i}, \mathbb{R}^{3}\right)$ such that

$$
\int_{\Omega_{i}} \varphi\left(\mathbf{F}+\nabla \mathbf{u}^{(h)}, \frac{\mathbf{z}_{p}}{\frac{d}{h}}\right) d \mathbf{z} \geqq \int_{\Omega_{i}} \varphi^{H}\left(\mathbf{F}+\nabla \mathbf{u}^{(h)}+\nabla \mathbf{v}_{h}^{i}\right) d \mathbf{z}-\delta,
$$

and

$$
\int_{\Omega_{i}}\left|\mathbf{v}_{h}^{i}\right|^{p} d \mathbf{z} \leqq \delta^{p}
$$

By using a cut-off argument as above (see Chapter 24 of [14]), fixed $t>0$ and $\sigma>0$ we can find a cut-off function $\eta_{j}^{i}$ between $i+(t, 1-t)^{2}$ and $i+Z$ and a positive constant $c(t, \sigma)$ such that if we set $\mathbf{w}_{j}^{i}=\mathbf{u}^{(h)}+\eta_{h}^{i} \mathbf{v}_{h}^{i}, Z_{t}=(0,1)^{2} \backslash(t, 1-t)^{2}$ and $\Omega_{i}^{t}=Z_{t} \times(0,1)$, we have

$$
\begin{aligned}
& \int_{\Omega_{i}} \varphi^{H}\left(\mathbf{F}+\nabla \mathbf{w}_{h}^{i}\right) d \mathbf{z} \\
& \leqq(1+\sigma)\left(\int_{\Omega_{i}} \varphi^{H}\left(\mathbf{F}+\nabla \mathbf{u}^{(h)}+\nabla \mathbf{v}_{h}^{i}\right) d \mathbf{z}+\int_{\Omega_{i}^{t}} \varphi^{H}\left(\mathbf{F}+\nabla \mathbf{u}^{(h)}\right) d \mathbf{z}\right) \\
&+c(t, \sigma) \int_{\Omega_{i}^{t}}\left|\mathbf{v}_{h}^{i}\right|^{p} d \mathbf{z}+\sigma \\
& \leqq(1+\sigma)\left(\int_{\Omega_{i}} \varphi^{H}\left(\mathbf{F}+\nabla \mathbf{u}^{(h)}+\nabla \mathbf{v}_{h}^{i}\right) d \mathbf{z}\right. \\
&\left.+2^{p} c_{2} \int_{\Omega_{i}^{t}}\left(1+|\mathbf{F}|^{p}+\left|\nabla \mathbf{u}^{(h)}\right|^{p}\right) d x\right)+c(t, \sigma) \delta^{p}+\sigma .
\end{aligned}
$$


If we define

$$
\tilde{\mathbf{u}}^{(h)}= \begin{cases}\mathbf{w}_{h}^{i} & \text { on } \Omega_{i}, \quad i \in I_{h}^{1}, \\ \mathbf{u}^{(h)} & \text { otherwise, }\end{cases}
$$

then we have

$$
\begin{aligned}
& \int_{\left(0, T_{h}\right)^{2} \times(0,1)} \varphi^{H}\left(\mathbf{F}+\nabla \tilde{\mathbf{u}}^{(h)}\right) d \mathbf{z} \\
& \leqq \sum_{i \in I_{h}^{1}} \int_{\Omega_{i}} \varphi^{H}\left(\mathbf{F}+\nabla \mathbf{w}_{j}^{i}\right) d \mathbf{z}+\sum_{i \in I_{h}^{2}} \int_{\Omega_{i}} \varphi^{H}\left(\mathbf{F}+\nabla \mathbf{u}^{(h)}\right) d \mathbf{z}+\int_{C_{h}} \varphi^{H}(\mathbf{F}) d \mathbf{z} \\
& \leqq \\
& \quad \sum_{i \in I_{h}^{1}} \int_{\Omega_{i}} \varphi^{H}\left(\mathbf{F}+\nabla \mathbf{w}_{j}^{i}\right) d \mathbf{z}+2^{p} c_{2} \int_{D_{h} \times(0,1)}\left(1+|\overline{\mathbf{F}}|^{p}+\left|\nabla \mathbf{u}^{(h)}\right|^{p}\right) d \mathbf{z} \\
& \quad+8 T_{h} c_{2}\left(1+|\overline{\mathbf{F}}|^{p}\right),
\end{aligned}
$$

where

$$
D_{h}=\bigcup_{i \in I_{h}^{2}}(i+Z) .
$$

Note that $h^{2}\left|D_{h}\right|=h^{2} \#\left(I_{h}^{2}\right)=o(1)$ as $M \rightarrow+\infty$.

By (6.11)-(6.14) we get

$$
\begin{aligned}
& \int_{\left(0, T_{h}\right)^{2} \times(0,1)} \varphi^{H}\left(\mathbf{F}+\nabla \tilde{\mathbf{u}}^{(h)}\right) d \mathbf{z} \\
& \leqq \sum_{i \in I_{h}^{1}}\left\{( 1 + \sigma ) \left(\int_{\Omega_{i}} \varphi^{H}\left(\mathbf{F}+\nabla \mathbf{u}^{(h)}+\nabla \mathbf{v}_{h}^{i}\right) d \mathbf{z}\right.\right. \\
& \left.\left.+2^{p} c_{2} \int_{\Omega_{i}^{t}}\left(1+|\overline{\mathbf{F}}|^{p}+\left|\nabla \mathbf{u}^{(h)}\right|^{p}\right) d \mathbf{z}\right)+c(t, \sigma) \delta^{p}+\sigma\right\} \\
& +2^{p} c_{2} \int_{D_{h} \times(0,1)}\left(1+|\overline{\mathbf{F}}|^{p}+\left|\nabla \mathbf{u}^{(h)}\right|^{p}\right) d \mathbf{z}+8 T_{h} c_{2}\left(1+|\overline{\mathbf{F}}|^{p}\right) \\
& \leqq \sum_{i \in I_{h}^{1}}\left\{( 1 + \sigma ) \left(\int_{\Omega_{i}} \varphi\left(\mathbf{F}+\nabla \mathbf{u}^{(h)}, \frac{\mathbf{z}_{p}}{\frac{d}{h}}\right) d \mathbf{z}\right.\right. \\
& \left.\left.+2^{p} c_{2} \int_{\Omega_{i}^{t}}\left(1+|\overline{\mathbf{F}}|^{p}+\left|\nabla \mathbf{u}^{(h)}\right|^{p}\right) d \mathbf{z}\right)+c(t, \sigma) \delta^{p}+\sigma+\delta\right\} \\
& +2^{p} c_{2} \int_{D_{h} \times(0,1)}\left(1+|\overline{\mathbf{F}}|^{p}+\left|\nabla \mathbf{u}^{(h)}\right|^{p}\right) d \mathbf{z}+8 T_{h} c_{2}\left(1+|\overline{\mathbf{F}}|^{p}\right) \\
& \leqq(1+\sigma)\left\{\int_{\left(0, T_{h}\right)^{2} \times(0,1)} \varphi\left(\mathbf{F}+\nabla \mathbf{u}^{(h)}, \frac{\mathbf{z}_{p}}{\frac{d}{h}}\right) d \mathbf{z}\right. \\
& \left.+2^{p} c_{2} \int_{\left(D_{h} \cup E_{h}\right) \times(0,1)}\left(1+|\overline{\mathbf{F}}|^{p}+\left|\nabla \mathbf{u}^{(h)}\right|^{p}\right) d \mathbf{z}\right\} \\
& +\left(c(t, \sigma) \delta^{p}+\sigma+\delta\right) T_{h}^{2}+8 T_{h} c_{2}\left(1+|\overline{\mathbf{F}}|^{p}\right),
\end{aligned}
$$


where

$$
E_{h}=\bigcup_{i \in I_{h}^{1}}\left(i+Z_{t}\right)
$$

Note that $\left|E_{h}\right| \leqq 4 t T_{h}^{2}$.

Changing back variables we obtain

$$
\begin{aligned}
& \frac{1}{T_{h}^{2}} \int_{\left(0, T_{h}\right)^{2} \times(0,1)} \varphi^{H}\left(\mathbf{F}+\nabla \tilde{\mathbf{u}}^{(h)}\right) d \mathbf{z} \\
\leqq & (1+\sigma)\left\{\int_{(0,1)^{3}} \varphi\left(\overline{\mathbf{F}}+\nabla_{p} \boldsymbol{\omega}^{(h)} \mid \frac{1}{h} \boldsymbol{\omega}_{, 3}^{(h)}, \frac{\mathbf{z}_{p}}{d}\right) d \mathbf{z}\right. \\
& \left.+2^{p} c_{2} \int_{\left(h D_{h} \cup h E_{h}\right) \times(0,1)}\left(1+|\overline{\mathbf{F}}|^{p}+\left.\left|\nabla_{p} \boldsymbol{\omega}^{(h)}\right| \frac{1}{h} \boldsymbol{\omega}_{, 3}^{(h)}\right|^{p}\right) d \mathbf{z}\right\} \\
& +c(t, \sigma) \delta^{p}+\sigma+\delta+8 h c_{2}\left(1+|\overline{\mathbf{F}}|^{p}\right) .
\end{aligned}
$$

By the equi-integrability of $\left(\left.\left|\nabla_{p} \omega^{(h)}\right| \frac{1}{h} \omega_{, 3}^{(h)}\right|^{p}\right)$ we have

$$
2_{\left(h D_{h} \cup h E_{h}\right) \times(0,1)}\left(1+|\overline{\mathbf{F}}|^{p}+\left.\left|\nabla_{p} \boldsymbol{\omega}^{(h)}\right| \frac{1}{h} \boldsymbol{\omega}_{, 3}^{(h)}\right|^{p}\right) d \mathbf{z} \leqq \zeta\left(\left|h D_{h} \cup h E_{h}\right|\right),
$$

where $\lim _{s \rightarrow 0^{+}} \zeta(s)=0$. Note that $\left|h D_{h} \cup h E_{h}\right| \leqq c\left(\frac{1}{M}+t\right)$ for some constant $c$ independent of $h$. Letting $h \rightarrow 0$, we get

$$
\begin{aligned}
Q \varphi_{0}^{H}(\overline{\mathbf{F}}) \leqq & \liminf _{h \rightarrow 0} \frac{1}{T_{h}^{2}} \int_{\left(0, T_{h}\right)^{2} \times(0,1)} \varphi^{H}\left(\mathbf{F}+\nabla \tilde{\mathbf{u}}^{(h)}\right) d \mathbf{z} \\
\leqq & (1+\sigma) \liminf _{h \rightarrow 0} e_{1}^{(h)}\left[\mathbf{y}^{(h)}\right]+(1+\sigma) \zeta\left(\left|h D_{h} \cup h E_{h}\right|\right) \\
& +c(t, \sigma) \delta^{p}+\sigma+\delta .
\end{aligned}
$$

Letting, in the following order, $\delta \rightarrow 0, M \rightarrow+\infty, t \rightarrow 0, \sigma \rightarrow 0$, we obtain the desired lower bound.

We now construct a recovery sequence for any $\mathbf{y} \in V_{S}$. From Lemma 2.1 in [36], the bulk homogenized energy density $\varphi^{H}$ satisfies the same growth and Lipschitz conditions (2.4)-(2.5) on $\varphi$ (see also BRAIDES \& DEFRANCESCHI [14]). It follows that $\varphi_{0}^{H}$ given by $(6.3)_{2}$ is well defined and the infimum is achieved. Further, Proposition 1 of LE DreT \& RAOUlt [21] shows that $\varphi_{0}^{H}(\overline{\mathbf{F}})$ satisfies the growth and coercivity estimates (2.4) and is continuous. Therefore, the relaxation theorem of DACOROGNA [19] yields the existence of a sequence $\mathbf{y}^{(\delta)}$ which converges weakly to $\mathbf{y}$ in $W^{1, p}\left(S, \mathbb{R}^{3}\right)$ such that

$$
\int_{S} \varphi_{0}^{H}\left(\nabla_{p} \mathbf{y}^{(\delta)}\right) d \mathbf{z}_{p} \rightarrow \int_{S} Q \varphi_{0}^{H}\left(\nabla_{p} \mathbf{y}\right) d \mathbf{z}_{p} \quad \text { as } \delta \rightarrow 0 .
$$


The Lipschitz domain $S$ permits a sequence of piecewise affine functions $\mathbf{y}^{(\delta, \varepsilon)} \rightarrow$ $\mathbf{y}^{(\delta)}$ in $W^{1, p}\left(S, \mathbb{R}^{3}\right)$ as $\varepsilon \rightarrow 0$ for fixed $\delta$ such that

$$
\int_{S} \varphi_{0}^{H}\left(\nabla_{p} \mathbf{y}^{(\delta, \varepsilon)}\right) d \mathbf{z}_{p} \rightarrow \int_{S} \varphi_{0}^{H}\left(\nabla_{p} \mathbf{y}^{(\delta)}\right) d \mathbf{z}_{p} \quad \text { as } \varepsilon \rightarrow 0 .
$$

Let $\left\{S_{i}\right\}$ be a finite partition of $S$ into open sets $S_{i}$ (except for a set of measure zero) such that $\nabla_{p} \mathbf{y}^{(\delta, \varepsilon)}=\overline{\mathbf{F}}_{i}^{(\delta, \varepsilon)}$ constant for $\mathbf{z}_{p} \in S_{i}$. Further, there exists a constant vector $\mathbf{b}_{i}^{(\delta, \varepsilon)}$ such that

$$
\varphi_{0}^{H}\left(\overline{\mathbf{F}}_{i}^{(\delta, \varepsilon)}\right)=\varphi^{H}\left(\overline{\mathbf{F}}_{i}^{(\delta, \varepsilon)} \mid \mathbf{b}_{i}^{(\delta, \varepsilon)}\right)
$$

for each $i$. Let $\mathbf{F}_{i}^{(\delta, \varepsilon)}=\left(\overline{\mathbf{F}}_{i}^{(\delta, \varepsilon)} \mid \mathbf{b}_{i}^{(\delta, \varepsilon)}\right)$. On each subdomain $S_{i}$, using the definition of $\varphi^{H}(6.3)_{3}$, there exists $\omega_{i}^{(\delta, \varepsilon, \eta)} \in W_{0}^{1, p}\left(k^{(\eta)} \hat{Z}, \mathbb{R}^{3}\right)$ such that

$$
\frac{1}{k^{(\eta)^{3}}} \int_{k^{(\eta)} \hat{Z}} \varphi\left(\mathbf{F}_{i}^{(\delta, \varepsilon)}+\nabla \omega_{i}^{(\delta, \varepsilon, \eta)}, \mathbf{z}_{p}\right) d \mathbf{z} \rightarrow \varphi^{H}\left(\mathbf{F}_{i}^{(\delta, \varepsilon)}\right) \quad \text { as } \eta \rightarrow 0,
$$

where $\hat{Z}=(0,1)^{3}$ and $k^{(\eta)} \in \mathbb{N}$. Further, let $\tilde{\mathbf{b}}_{i}^{(\delta, \varepsilon, \eta)} \in C_{0}^{\infty}\left(S_{i}, \mathbb{R}^{3}\right)$ be such that $\tilde{\mathbf{b}}_{i}^{(\delta, \varepsilon, \eta)} \rightarrow \mathbf{b}_{i}^{(\delta, \varepsilon)}$ in $L^{p}\left(S_{i}, \mathbb{R}^{3}\right)$ as $\eta \rightarrow 0$. Now on each subdomain $S_{i}$, consider the sequence

$$
\tilde{\mathbf{y}}_{i}^{(\delta, \varepsilon, \eta, h)}(\mathbf{z})=\mathbf{y}^{(\delta, \varepsilon)}\left(\mathbf{z}_{p}\right)+h \tilde{\mathbf{b}}_{i}^{(\delta, \varepsilon, \eta)}\left(\mathbf{z}_{p}\right) z_{3}+d \boldsymbol{\omega}_{i}^{(\delta, \varepsilon, \eta)}\left(\frac{\mathbf{z}_{p}}{d}, \frac{h}{d} z_{3}\right) .
$$

Using the coercivity estimate (2.4) and the Lipschitz conditions (2.5) on $\varphi$ and recalling (6.19), we have

$$
\int_{S_{i} \times(0,1)} \varphi\left(\nabla_{p} \tilde{\mathbf{y}}_{i}^{(\delta, \varepsilon, \eta, h)} \mid \frac{1}{h} \tilde{\mathbf{y}}_{i, 3}^{(\delta, \varepsilon, \eta, h)}, \frac{\mathbf{z}_{p}}{d}\right) d \mathbf{z} \rightarrow \int_{S_{i} \times(0,1)} \varphi^{H}\left(\mathbf{F}_{i}^{(\delta, \varepsilon)}\right) d \mathbf{z}
$$

as $h \rightarrow 0$ first, and then $\eta \rightarrow 0$. Further appealing to a standard diagonalization process and a similar argument used in Lemma 2.1.(a) of MüLLER [36], we can construct a sequence $\tilde{\mathbf{y}}_{i}^{(\delta, \varepsilon, \eta(h), h)}$ such that

$$
\left.\left(\tilde{\mathbf{y}}_{i}^{(\delta, \varepsilon, \eta(h), h)}-\mathbf{y}^{(\delta, \varepsilon)}\right)\right|_{\partial S_{i} \times(0,1)}=0
$$

and (6.21) holds for this sequence $\tilde{\mathbf{y}}_{i}^{(\delta, \varepsilon, \eta(h), h)}$. Now extend $\left(\tilde{\mathbf{y}}_{i}^{(\delta, \varepsilon, \eta(h), h)}-\mathbf{y}^{(\delta, \varepsilon)}\right)$ by zero in $\left(\mathbb{R}^{2} \backslash S_{i}\right) \times(0,1)$ and define

$$
\mathbf{y}^{(\delta, \varepsilon, \eta(h), h)}=\mathbf{y}^{(\delta, \varepsilon)}+\sum_{i}\left(\tilde{\mathbf{y}}_{i}^{(\delta, \varepsilon, \eta(h), h)}-\mathbf{y}^{(\delta, \varepsilon)}\right) .
$$

Set $\mathbf{b}^{(\delta, \varepsilon)}\left(\mathbf{z}_{p}\right)=\sum_{i} \mathbf{b}_{i}^{(\delta, \varepsilon)} \chi_{S_{i}} \in L^{p}\left(S, \mathbb{R}^{3}\right)$ where $\chi$ is the characteristic function. From (6.18), (6.21) and (6.22), we have

$$
\begin{aligned}
\int_{\Omega^{1}} \varphi\left(\nabla_{p} \mathbf{y}^{(\delta, \varepsilon, \eta(h), h)} \mid \frac{1}{h} \mathbf{y}_{, 3}^{(\delta, \varepsilon, \eta(h), h)}, \frac{\mathbf{z}_{p}}{d}\right) d \mathbf{z} & \rightarrow \int_{\Omega^{1}} \varphi^{H}\left(\nabla_{p} \mathbf{y}^{(\delta, \varepsilon)} \mid \mathbf{b}^{(\delta, \varepsilon)}\right) d \mathbf{z} \\
& =\int_{S} \varphi_{0}^{H}\left(\nabla_{p} \mathbf{y}^{(\delta, \varepsilon)}\right) d \mathbf{z}_{p}
\end{aligned}
$$


as $h \rightarrow 0$. Let $\varepsilon \rightarrow 0$ first and $\delta \rightarrow 0$ next in (6.17) and (6.16) and recall (6.23). We obtain the desired recovery sequence by further appealing to a standard diagonalization argument on $\mathbf{y}^{(\delta, \varepsilon, \eta(h), h)}$ (see, e.g., Aтtouch [4, Corollary 1.16]).

If $\kappa \neq 0$ and $\frac{\kappa}{d} \rightarrow \alpha<\infty$ as $h \rightarrow 0$, then using the same change of variables (6.1) above, we can rewrite $e_{1}^{(h)}$ as

$$
h^{2} \int_{0}^{1} \int_{\frac{S}{h}}\left\{\left(\frac{\kappa}{d}\right)^{2}\left(\frac{d}{h}\right)^{2}\left|\nabla^{2} \hat{\mathbf{y}}\right|^{2}+\varphi\left(\nabla \hat{\mathbf{y}}, \frac{\hat{\mathbf{z}}_{p}}{\frac{d}{h}}\right)\right\} d \hat{\mathbf{z}}_{p} d \hat{z}_{3} .
$$

Comparing (6.24) with equation (2.4) and $\gamma=1$ in [24] suggests that $\bar{\varphi}$ is obtained by averaging and homogenizing the bulk heterogeneous material first, and then passing to the thin-film limit, or

$$
\begin{aligned}
\bar{\varphi}(\overline{\mathbf{F}}) & =Q \varphi_{0}^{A H}(\overline{\mathbf{F}}), \\
\varphi_{0}^{A H}(\overline{\mathbf{F}}) & =\inf _{\mathbf{b} \in \mathbb{R}^{3}} \varphi^{A H}(\overline{\mathbf{F}} \mid \mathbf{b}), \\
\varphi^{A H}(\mathbf{F}) & =\inf _{k \in \mathbb{N}} \inf _{\boldsymbol{\omega} \in W_{0}^{1, p}(k \hat{Z}) \cap W_{0}^{2,2}(k \hat{Z})} f_{k \hat{Z}}\left\{\alpha^{2}\left|\nabla^{2} \boldsymbol{\omega}\right|^{2}+\varphi\left(\mathbf{F}+\nabla \boldsymbol{\omega}, \mathbf{z}_{p}\right)\right\} d \mathbf{z} .
\end{aligned}
$$

It can be shown easily that Theorem 5 still holds if $\alpha=0$. For $\alpha>0$, we conjecture that (6.25) can be confirmed by following the approach similar to that of Theorem 5 if the appropriate version of Lemma 2 can be established.

Finally, our Theorem 1(i) includes the case $\varphi=\varphi\left(\mathbf{F}, \frac{\mathbf{z}_{p}}{d}\right), \frac{\kappa}{d} \rightarrow \alpha=\infty$ as $h \rightarrow 0$. Note that the ratio of $\kappa$ and $h$ turns out to be irrelevant in this case (see Remark 6).

\section{Applications}

\subsection{Shape-memory effect in polycrystalline thin films}

Shape-memory effect is a phenomenon observed in certain martensitic materials where deformation suffered below a critical temperature is recovered on heating. The source of the shape-memory effect is the martensitic phase transformation. The most characteristic observable feature of a martensitic phase transformation is the microstructure it generates. BALL \& JAMES [5,6] have proposed a theoretical framework to model these fine phase mixtures based on the minimization of the free energy. According to this theory, the energy density $\varphi$ has a multi-well structure, and each well represents a phase or variant. The strains that can be recovered in the shape-memory effect are exactly those that can be attained by the rearrangement of coherent martensitic variants. Thus, they are contained in a set on which the relaxation (quasi-convexification) of $\varphi$ vanishes in a single crystal. BHATTACHARYA $\&$ KoHN [11] have extended this theory to polycrystalline bulk martensites by using the framework of nonlinear homogenization. A polycrystal is an aggregate of a great number of single crystal grains with different orientations. The texture of a 
polycrystal (the orientation of the grains) is described by a rotation-valued function $\mathbf{R}(\mathbf{x}): \Omega \rightarrow \mathrm{SO}(3)$. When subjected to deformation $\mathbf{y}(\mathbf{x})$, the total energy stored in this polycrystal is

$$
\int_{\Omega} \varphi(\nabla \mathbf{y}(\mathbf{x}), \mathbf{x}) d \mathbf{x}=\int_{\Omega} \varphi(\nabla \mathbf{y}(\mathbf{x}) \mathbf{R}(\mathbf{x})) d \mathbf{x} .
$$

The strains recoverable on heating in a polycrystal are contained in a set on which this energy vanishes.

Our main interest is the study of shape-memory behavior in a polycrystalline thin film. Specifically, we want to find all possible deformations that can be recovered on heating for this film. In bulk polycrystals, there are several important factors influencing the class of recoverable strains and we refer readers to BHATTACHARYA $\&$ KOHN [10,11] and SHU \& BHATTACHARYA [37]. In thin films, we now show that this class can also crucially depend on the ratio of different length scales.

7.1.1. Model example. We consider a two-dimensional model problem where the deformation $\mathbf{y}$ is replaced by a scalar-valued function $\eta: \mathbb{R}^{2} \rightarrow \mathbb{R}$ and $\mathbf{R}: \mathbb{R} \rightarrow$ $\mathrm{SO}(2)$ represents the texture of our polycrystal film. We assume $\mathbf{R}\left(z_{1}\right)$ is periodic with period $[0,1]$. The microscopic energy density has the form

$$
\varphi\left(\mathbf{f}, \frac{z_{1}}{d}\right)=\varphi\left(\mathbf{R}^{T}\left(\frac{z_{1}}{d}\right) \mathbf{f}\right) .
$$

The total energy of the film per unit thickness is

$$
\begin{aligned}
e_{1}^{(h)}[\eta]=\int_{\Omega^{1}}\{ & \kappa^{2}\left(\left|\frac{\partial^{2} \eta}{\partial z_{1}^{2}}\right|^{2}+\frac{2}{h^{2}}\left|\frac{\partial \eta}{\partial z_{1}} \frac{\partial \eta}{\partial z_{2}}\right|^{2}+\frac{1}{h^{4}}\left|\frac{\partial^{2} \eta}{\partial z_{2}^{2}}\right|^{2}\right) \\
& \left.+\varphi\left(\frac{\partial \eta}{\partial z_{1}} \mid \frac{1}{h} \frac{\partial \eta}{\partial z_{2}}, \frac{z_{1}}{d}\right)\right\} d \mathbf{z}
\end{aligned}
$$

where $S=(0, L)$ is the one-dimensional film and $\Omega^{1}=S \times(0,1)$. The limiting energy per unit thickness is

$$
e_{1}^{(0)}[\eta]=\int_{S} \bar{\varphi}\left(\frac{\partial \eta}{\partial z_{1}}\right) d z_{1} .
$$

Assume the film thickness is comparable to the grain size and the interfacial energy is negligible, i.e., $\frac{h}{d} \rightarrow \beta>0$ and $\frac{\kappa}{d} \rightarrow 0$ as $h \rightarrow 0$. It follows from (5.1) that $e_{1}^{(h)}$ $\Gamma$-converges to the functional $e_{1}^{(0)}$ if the effective energy density $\bar{\varphi}(\xi)$ of the film is given by

$$
\bar{\varphi}(\xi)=\inf _{k \in \mathbb{N}} \inf _{\boldsymbol{\omega} \in \hat{A}_{k}^{\beta}} f_{\Omega_{k}^{\beta}} \varphi\left(\mathbf{R}^{T}\left(z_{1}\right)\left(\xi+\frac{\partial \boldsymbol{\omega}}{\partial z_{1}} \mid \frac{\partial \boldsymbol{\omega}}{\partial z_{2}}\right)\right) d \mathbf{z}
$$

where

$$
\begin{aligned}
& \Omega_{k}^{\beta}=k Z \times(0, \beta), \quad Z=(0,1), \\
& \hat{A}_{k}^{\beta}=\left\{\omega: \omega \in W_{\text {per }}^{1, p}\left(\Omega_{k}^{\beta}, \mathbb{R}\right)\right\},
\end{aligned}
$$


where elements of $W_{\text {per }}^{1, p}\left(\Omega_{k}^{\beta}, \mathbb{R}\right)$ are periodic only in $z_{1}$ with period $k Z$. Notice that we have replace $\tilde{A}_{k}^{\beta}$ in (5.3) by $\hat{A}_{k}^{\beta}$ in (7.5); a slight refinement of the argument of MüLlER [36] shows we can minimize (7.3) over either of these two spaces.

Let $\hat{\varphi}$ be the relaxation of $\varphi$ and consider the analogue of (7.3)

$$
\inf _{k \in \mathbb{N}} \inf _{\boldsymbol{\omega} \in \hat{A}_{k}^{\beta}} f_{\Omega_{k}^{\beta}} \hat{\varphi}\left(\mathbf{R}^{T}\left(z_{1}\right)\left(\xi+\frac{\partial \boldsymbol{\omega}}{\partial z_{1}} \mid \frac{\partial \boldsymbol{\omega}}{\partial z_{2}}\right)\right) d \mathbf{z} .
$$

These two expressions (7.3) and (7.6) are in some sense equivalent since the minimizing sequences for the former converge weakly to the minimizers of the latter, and the minimizers of the latter give rise to minimizing sequences for the former [2]. Therefore, from now on, we will not distinguish between minimizing sequences and minimizers but use $\hat{\varphi}$ in (7.6) to replace $\varphi$ in (7.3). Further, in our particular problem below, $\hat{\varphi}$ is convex and it suffices to take $k=1$ [36].

We state a useful lower bound for $\bar{\varphi}$. Let

$$
\psi^{*}(\mathbf{g})=\max _{\mathbf{f}}\{\mathbf{f} \cdot \mathbf{g}-\psi(\mathbf{f})\}
$$

be the Fenchel transformation of the function $\psi$. Let $\omega \in \hat{A}_{1}^{\beta}$ and consider any vector field $\mathbf{g}=\left(g_{1}, g_{2}\right)^{T}$ which is periodic in $z_{1}$ with period [0,1]. If we assume $\nabla \cdot \mathbf{g}=0$ in $\Omega_{1}^{\beta}$ and $\mathbf{g} \cdot \mathbf{n}=0$ at $z_{2}=0$ and $\beta$ where $\mathbf{n}=(0,1)^{T}$ is the outward normal, it follows that

$$
f_{\Omega_{1}^{\beta}} \hat{\varphi}\left(\mathbf{R}^{T}\left(f_{1}+\boldsymbol{\omega}_{, 1} \mid \boldsymbol{\omega}_{, 2}\right)\right), d \mathbf{z} \geqq f_{\Omega_{1}^{\beta}}\left\{f_{1} g_{1}-\hat{\varphi}^{*}\left(\mathbf{R}^{T} \mathbf{g}\right)\right\} d \mathbf{z} .
$$

Optimization over $\boldsymbol{\omega}$ and $\mathbf{g}$ gives

$$
\bar{\varphi}\left(f_{1}\right) \geqq \max _{\substack{\nabla \cdot \mathbf{g}=0 \\ \mathbf{g} \cdot \mathbf{n}=0 \text { on } z_{2}=0, \beta}} f_{\Omega_{1}^{\beta}}\left\{f_{1} g_{1}-\hat{\varphi}^{*}\left(\mathbf{R}^{T}\left(z_{1}\right) \mathbf{g}(\mathbf{z})\right)\right\} d \mathbf{z}
$$

where $\mathbf{g}$ is periodic in $z_{1}$ with period $[0,1]$.

We now specialize and consider a two-variant material with microscopic elastic energy

$$
\varphi(\mathbf{f})=\min \left\{\frac{1}{2}\left(\left(f_{1}-1\right)^{2}+f_{2}^{2}\right), \frac{1}{2}\left(\left(f_{1}+1\right)^{2}+f_{2}^{2}\right)\right\}
$$

where $\mathbf{f}=\left(f_{1}, f_{2}\right)^{T}$. It can be shown that, in this case,

$$
\hat{\varphi}(\mathbf{f})=\frac{1}{2}\left\{\left(\left|f_{1}\right|-1\right)_{+}^{2}+f_{2}^{2}\right\}
$$

with the convention that $a_{+}=\max \{a, 0\}$. The explicit expression of the Fenchel transform of $\hat{\varphi}$ is

$$
\hat{\varphi}^{*}(\mathbf{f})=\frac{1}{2}|\mathbf{f}|^{2}+\left|f_{1}\right|
$$


Our main interest is the study of the shape-memory behavior or stress-free configuration of the film. In other words, we want to determine the "set of recoverable strains for the polycrystalline thin film" which we define as (c.f. [11])

$$
\mathcal{P}_{f}=\{\xi: \bar{\varphi}(\xi)=0\} .
$$

A single crystal is the special case of the polycrystal. In that case, clearly, $\mathcal{P}$ contains a line segment $[-1,1]$. But for films with general texture, $\mathcal{P}$ is expected to be smaller than this line segment. The extent of this reduction of recoverable strains in thin films also depends on the parameter $\beta$ and our task is to determine it. We do so in detail for the special polycrystal shown in Fig. 3(a).

Proposition 1. Consider the polycrystal with the texture

$$
\mathbf{R}\left(z_{1}\right)=\left\{\begin{array}{lll}
\mathbf{R}_{1} & m \leqq z_{1}<m+\frac{1}{2} & \text { (“grain 1,”) } \\
\mathbf{R}_{2} & m-\frac{1}{2} \leqq z_{1}<m & \text { ("grain 2,”) }
\end{array}\right.
$$

where $m \in \mathbb{Z}$ and

$$
\mathbf{R}_{1}=\left(\begin{array}{cc}
\cos \theta & -\sin \theta \\
\sin \theta & \cos \theta
\end{array}\right) \quad \text { and } \quad \mathbf{R}_{2}=\left(\begin{array}{cc}
\cos \theta & \sin \theta \\
-\sin \theta & \cos \theta
\end{array}\right)
$$

in the "grey" and "white" rectangles of Fig. 3(a) for $0<\theta<\frac{\pi}{2}$. If $\beta \leqq \frac{1}{2} \cot \theta$, then

$$
\mathcal{P}=\{\xi:|\xi| \leqq \cos \theta-2 \beta \sin \theta\}
$$

if $\beta=\frac{1}{2} \cot \theta$, then there exist positive constants $c$ and $C$ such that

$$
c|\xi|^{3} \leqq \bar{\varphi}(\xi) \leqq C|\xi|^{3} \quad \text { for } \xi \text { sufficiently small }
$$

and clearly $\mathcal{P}=\{0\} ;$ finally, if $\beta>\frac{1}{2} \cot \theta$, then there exists a constant $C>0$ such that

$$
\bar{\varphi}(\xi) \geqq C|\xi|^{2}
$$

and once again $\mathcal{P}=\{0\}$.

Proof. Consider the case $\beta \leqq \frac{1}{2} \cot \theta$ first. We wish to construct a test function $\eta$ with zero energy. This requires $\mathbf{R}^{T} \nabla \eta$ to be parallel to $\mathbf{e}_{1}$ a.e. and $|\nabla \eta| \leqq 1$. This motivates the following construction:

$$
\nabla \eta= \begin{cases}a(\cos \theta, \sin \theta)^{T} & \text { in the darkly shaded region of "grain 1," } \\ a(\cos \theta,-\sin \theta)^{T} & \text { in the darkly shaded region of "grain 2," } \\ 0 & \text { otherwise, }\end{cases}
$$

as shown in Fig. 3(b) for some $|a| \leqq 1$. This test field is compatible since $\nabla \eta$ is piecewise constant, and on every interface $[[\nabla \eta]] \cdot \hat{\mathbf{t}}=0$ where [[ ]] denotes the jump across the interface and $\hat{\mathbf{t}}$ is the tangent. Furthermore, the area of support of $\nabla \eta$ 


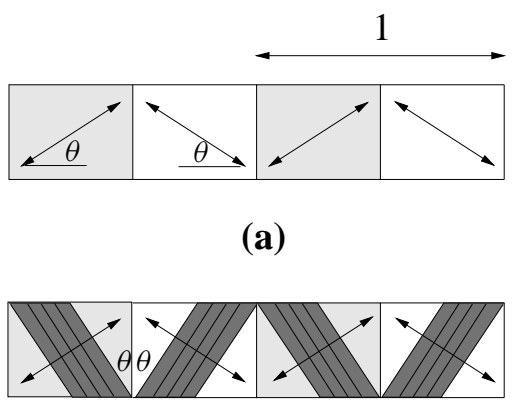

(b)

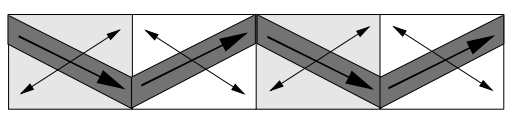

(c)

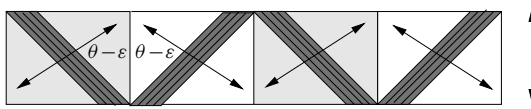

(d)

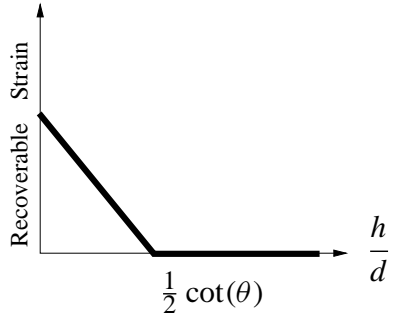

(e)

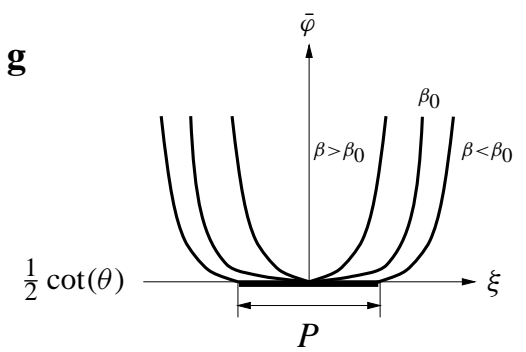

(f)

Fig. 3. (a) A polycrystalline thin film with the texture containing two orientations. (b) The test field $\nabla \eta$. The darkly shaded regions form the support of $\nabla \eta$ and the straight lines within them are level sets of $\eta$. (c) The test divergence-free flow $\mathbf{g}$. The darkly shaded strips form the support of $\mathbf{g}$ and the arrows within them point out the direction of the flow. (d) The test field $\nabla \eta$ when $\beta=\beta_{0}=\frac{1}{2} \cot \theta$. The darkly shaded regions form the support of $\nabla \eta$ and the straight lines within them are level sets of $\eta$. (e) The recoverable strain $\left(=\frac{1}{2}|\mathcal{P}|\right)$ versus different values $\beta$. (f) The behavior of $\bar{\varphi}(\xi)$ for $\xi$ near zero with different values $\beta$. Notice that $\bar{\varphi}(\xi)$ grows quadratically if $\beta>\beta_{0}$, becomes flat (zero) if $\beta<\beta_{0}$, and has an exact cubic growth near the origin if $\beta=\beta_{0}$.

is greater than zero since $\beta \leqq \frac{1}{2} \cot \theta$. The average $f \nabla \eta=a(\cos \theta-2 \beta \sin \theta) \mathbf{e}_{1}$ and hence we obtain a lower bound of

$$
\mathcal{P} \supset\{\xi:|\xi| \leqq \cos \theta-2 \beta \sin \theta\}
$$

Next we show that $\mathcal{P}$ contains no other points. Indeed, this is equivalent to showing that $\bar{\varphi}(\xi)>0$ if $|\xi|>\cos \theta-2 \beta \sin \theta$. We can accomplish this using the lower bound (7.7). Consider the dark strips shown in Fig. 3(c). Taking the slopes of these strips as $\pm(\beta-\mu) /\left(\frac{1}{2}+\mu\right)$ where $\mu>0$, set

$$
\mathbf{g}= \begin{cases}\left(\frac{1}{2}+\mu, \mu-\beta\right)^{T} & \text { in the darkly shaded strip of "grain 1", } \\ \left(\frac{1}{2}+\mu, \beta-\mu\right)^{T} & \text { in the darkly shaded strip of "grain 2", } \\ 0 & \text { otherwise. }\end{cases}
$$


It is easy to verify that $\nabla \cdot \mathbf{g}=0$ and $\mathbf{g} \cdot \mathbf{n}=0$ at $z_{2}=0$ and $\beta$. Substituting the test field $\tau \mathbf{g}$ into (7.7) and using the formula (7.10) for $\hat{\varphi}^{*}$, we obtain

$$
\begin{gathered}
\bar{\varphi}\left(f_{1}\right) \geqq \tau f f_{1} g_{1}-\frac{\tau^{2}}{2} f|\mathbf{g}|^{2}-|\tau| f\left|\mathbf{e}_{1} \cdot \mathbf{R}^{T}\left(z_{1}\right) \mathbf{g}\right| \\
=\frac{A}{2}\left\{-\left[\left(\frac{1}{2}+\mu\right)^{2}+(\mu-\beta)^{2}\right] \tau^{2}+(1+2 \mu) f_{1} \tau\right. \\
\left.-\left[(\cos \theta-2 \beta \sin \theta)+2 \mu c_{0}\right]|\tau|\right\},
\end{gathered}
$$

where $A=\frac{\mu(1+2 \beta)}{\beta(1+2 \mu)}$ is the area fraction of the strips and $c_{0}=\sin \theta+\cos \theta$. To get the optimal lower bound we maximize (7.16) over $\tau$. It follows that $\bar{\varphi}\left(f_{1}\right)>0$ if

$$
(1+2 \mu)\left|f_{1}\right|-\left[(\cos \theta-2 \beta \sin \theta)+2 \mu c_{0}\right]>0 .
$$

Notice that if $\left|f_{1}\right|>(\cos \theta-2 \beta \sin \theta)$, then the above inequality holds for sufficiently small $\mu$; and hence $f_{1} \notin \mathcal{P}$. This proves (7.11).

We now turn to the case $\beta>\frac{1}{2} \cot \theta$. We obtain the desired lower bound from (7.7) by using the same test field $\tau \mathbf{g}$ as above with

$$
\mu=\frac{2 \beta \sin \theta-\cos \theta}{2(\sin \theta+\cos \theta)}>0 .
$$

Then (7.16) gives

$$
\bar{\varphi}\left(f_{1}\right) \geqq \frac{A}{2}\left\{-\left[\left(\frac{1}{2}+\mu\right)^{2}+(\mu-\beta)^{2}\right] \tau^{2}+(1+2 \mu) f_{1} \tau\right\} .
$$

Maximizing over $\tau$, we conclude that for some $C>0$ independent of $f_{1}$,

$$
\bar{\varphi}\left(f_{1}\right) \geqq C f_{1}^{2} .
$$

Finally, we come to the case $\beta=\frac{1}{2} \cot \theta$. We start with the lower bound first. Using the divergence-free field $\tau \mathbf{g}$ in (7.15) and substituting it into (7.7), we have

$$
\bar{\varphi}\left(f_{1}\right) \geqq \frac{A}{2}\left\{-\left[\left(\frac{1}{2}+\mu\right)^{2}+(\mu-\beta)^{2}\right] \tau^{2}+(1+2 \mu) f_{1} \tau-2 \mu c_{0}|\tau|\right\},
$$

where $c_{0}=\sin \theta+\cos \theta$. This bound is positive if $(1+2 \mu)\left|f_{1}\right|>2 \mu c_{0}$ or

$$
\mu<\frac{\left|f_{1}\right|}{2\left(c_{0}-\left|f_{1}\right|\right)} \text {. }
$$

Now maximizing (7.19) with respect to $\tau$ and choosing $\mu=a\left|f_{1}\right|$ with $a<\frac{1}{2 c_{0}}$, we have for some $c>0$

$$
\begin{aligned}
\bar{\varphi}\left(f_{1}\right) & \geqq \frac{\mu(1+2 \beta)}{2 \beta(1+2 \mu)}\left(\frac{\left(\left|f_{1}\right|+2 \mu\left(\left|f_{1}\right|-c_{0}\right)\right)^{2}}{4\left[\left(\frac{1}{2}+\mu\right)^{2}+(\mu-\beta)^{2}\right]}\right) \\
& =c\left|f_{1}\right|^{3}+O\left(f_{1}\right)^{4} \text { for } f_{1} \text { near } 0 .
\end{aligned}
$$


Next we return to the upper bound. We choose $\varepsilon>0$ such that $\theta-\varepsilon>0$ and construct a field $\eta$ as shown in Fig. 3(d):

$$
\nabla \eta=\left\{\begin{array}{c}
\frac{1}{\cos \varepsilon}(\cos (\theta-\varepsilon), \sin (\theta-\varepsilon))^{T} \\
\quad \text { in the darkly shaded region of "grain } 1, " \\
\frac{1}{\cos \varepsilon}(\cos (\theta-\varepsilon),-\sin (\theta-\varepsilon))^{T} \\
\text { in the darkly shaded region of "grain } 2, " \\
\text { otherwise. }
\end{array}\right.
$$

By geometry, the area fraction of these darkly shaded strips is

$$
A=1-2 \beta \tan (\theta-\varepsilon)=\frac{\sin \varepsilon}{\sin \theta \cos (\theta-\varepsilon)}
$$

since $\cos \theta=2 \beta \sin \theta$. It follows that

$$
f_{1}=f \nabla \eta=\frac{1}{\cos \varepsilon}(\cos (\theta-\varepsilon)-2 \beta \sin (\theta-\varepsilon))=\frac{\tan \varepsilon}{\sin \theta} .
$$

The local energy is

$$
\hat{\varphi}\left(\mathbf{R}^{T}\left(z_{1}\right) \nabla \eta\right)=\frac{1}{2} \tan ^{2} \varepsilon
$$

whenever $\nabla \eta \neq 0$. Therefore, this test field gives an upper bound

$$
\begin{aligned}
\bar{\varphi}\left(f_{1}\right) & \leqq \frac{1}{2}\left(\frac{\sin \varepsilon}{\sin \theta \cos (\theta-\varepsilon)}\right) \tan ^{2} \varepsilon \\
& \leqq \frac{\sin ^{2} \theta}{2 \cos (\theta-\varepsilon) \sqrt{1+\sin ^{2} \theta f_{1}^{2}}} f_{1}^{3} \\
& \leqq \frac{\sin ^{2} \theta}{2 \cos \theta} f_{1}^{3} \cdot
\end{aligned}
$$

This example shows quite dramatically the effect of $\beta$ (film thickness over the size of heterogeneity) on the shape-memory behavior. The recoverable strain $\frac{1}{2}|\mathcal{P}|$ versus $\beta$ is shown in Fig. 3(e). Further, the growth of $\bar{\varphi}(\xi)$ as it departs from $\mathcal{P}$ also significantly depends on the ratio $\beta$. As shown in Fig. 3(f), there exists a point $\beta_{0}=\frac{1}{2} \cot \theta$ such that for $\beta>\beta_{0}, \bar{\varphi}(\xi)$ grows quadratically and this film behaves like a linear elastic material without shape-memory effect; for $\beta<\beta_{0}, \bar{\varphi}(\xi)$ is flat (zero) and this film displays the shape-memory effect; for $\beta=\beta_{0}, \bar{\varphi}(\xi)$ has an exact cubic growth for $\xi$ near the origin and this film behaves like a nonlinear elastic material with soft modulus.

In this example we only dealt with a simple texture with two orientations. But we can generalize our method to a texture with $N$ orientations within the cell. The exact set of recoverable strains and the behavior of $\bar{\varphi}(\xi)$ for $\xi$ near the origin can also be predicted similarly. However, our current analysis is texture-dependent and it would be very useful to develop texture-independent bounds to explore the full range of behavior of a film. BHATTACHARYA \& KOHN [11] have used the translation method to predict the shape-memory behavior for bulk materials. Unfortunately, 
it does not work here. For example, consider the following identity which plays a central role in their analysis. If $\eta=\mathbf{f} \cdot \mathbf{z}$ and $\zeta=\mathbf{g} \cdot \mathbf{z}$ at $\partial \Omega_{1}^{\beta}$, then

$$
f \operatorname{det}(\nabla \eta, \nabla \zeta)=\operatorname{det}(\mathbf{f}, \mathbf{g})
$$

Unfortunately (7.21) does not necessarily hold if we only know $\eta$ and $\zeta$ on part of the boundary $\partial Z \times(0, \beta)$ rather than an entire boundary $\partial \Omega_{1}^{\beta}$. Notice that the test field $\omega$ in the definition of variational principle (7.3) is periodic only in $z_{1}$ and does not satisfy any boundary condition on the top and bottom parts of the film. This lack of information prevents an identity like (7.21) and we cannot use their method. Similarly, in the lower bound (7.7), the divergence-free flow $\mathbf{g}$ must meet an additional requirement $\mathbf{g} \cdot \mathbf{n}=0$ on the top and bottom parts of the film. This adds another difficulty in developing useful bounds.

7.1.2. Martensitic thin films. We now turn to the physically relevant problem in three dimensions using the geometrically linear theory of martensites. Let

$$
\varphi(\mathbf{F})=\varphi^{\operatorname{lin}}(\mathbf{E}[\mathbf{F}]),
$$

where

$$
\mathbf{E}=\frac{1}{2}\left(\mathbf{F}+\mathbf{F}^{T}\right)-\mathbf{I}
$$

is the linear strain and $\mathbf{I}$ is the identity matrix. To describe a martensite, the energy density is endowed with a multi-well structure - one well for each phase or variant, i.e.,

$$
\varphi^{\operatorname{lin}}(\mathbf{E})=\min _{i=1, \cdots, k}\left\{\frac{1}{2}\left|\mathbf{E}-\mathbf{E}^{(i)}\right|^{2}\right\}
$$

where $\mathbf{E}^{(i)}$ is the transformation strain for $i=1, \cdots, k$. Notice that we have restricted our analysis to some fixed temperature below the transformation temperature, and have assumed elastic constants are equal to the identity for simplicity.

Shape-memory thin films are often made by sputtering [27,28,39,33, 18, 17]. The grains in these films are typically columnar (e.g., see Fig. 2 of [28]). So we assume

$$
\varphi^{\operatorname{lin}}\left(\mathbf{E}, \frac{\mathbf{z}_{p}}{d}\right)=\varphi^{\operatorname{lin}}\left(\mathbf{R}^{T}\left(\frac{\mathbf{z}_{p}}{d}\right) \mathbf{E} \mathbf{R}\left(\frac{\mathbf{z}_{p}}{d}\right)\right),
$$

where $\mathbf{R}: \mathbb{R}^{2} \rightarrow \mathrm{SO}(3)$ is a given function describing texture and is assumed to be periodic with period $[0,1]^{2}$.

Let us consider the single crystal film first. Let $\Pi$ be the matrix that projects any $3 \times 3$ matrix into a $3 \times 2$ matrix:

$$
\Pi=\left(\begin{array}{ll}
1 & 0 \\
0 & 1 \\
0 & 0
\end{array}\right) .
$$


Then,

$$
\begin{aligned}
\varphi_{0}(\overline{\mathbf{F}}) & =\inf _{\mathbf{b} \in \mathbb{R}^{3}} \varphi^{\operatorname{lin}}\left(\begin{array}{c|c}
\frac{1}{2}\left(\overline{\mathbf{F}}_{p}+\overline{\mathbf{F}}_{p}^{T}\right)-\Pi^{T} \Pi & \frac{1}{2}\left(\mathbf{b}_{p}+\mathbf{F}_{3 p}\right) \\
\hline \frac{1}{2}\left(\mathbf{b}_{p}+\mathbf{F}_{3 p}\right)^{T} & b_{3}-1
\end{array}\right) \\
& =\inf _{\mathbf{a} \in \mathbb{R}^{3}} \varphi^{\operatorname{lin}}\left(\begin{array}{c|c}
\overline{\mathbf{E}} \mathbf{a}_{p} \\
\hline \mathbf{a}_{p}^{T} \mid a_{33}
\end{array}\right)=\varphi_{0}^{\operatorname{lin}(\overline{\mathbf{E}}),}
\end{aligned}
$$

where $\overline{\mathbf{F}}_{p}=\Pi^{T} \mathbf{F} \Pi, \overline{\mathbf{E}}=\Pi^{T} \mathbf{E} \Pi ; \mathbf{F}_{3 p}=\left(F_{31}, F_{32}\right)^{T}, \mathbf{b}_{p}=\left(b_{1}, b_{2}\right)^{T}$ and $\mathbf{a}_{p}=\left(a_{1}, a_{2}\right)^{T}$. For the multi-well energy density defined by (7.22), $\varphi_{0}^{\text {lin }}$ can be shown to be

$$
\begin{aligned}
\varphi_{0}^{\operatorname{lin}}(\overline{\mathbf{E}}) & =\min _{i=1, \cdots, k}\left\{\frac{1}{2}\left|\overline{\mathbf{E}}-\overline{\mathbf{E}}^{(i)}\right|^{2}\right\} \\
\overline{\mathbf{E}}^{(i)} & =\Pi^{T} \mathbf{E}^{(i)} \Pi .
\end{aligned}
$$

We define $\mathcal{S}_{f}$, the set of recoverable strains in a single crystal film with orientation $\mathbf{e}_{3}$, to be

$$
\mathcal{S}_{f}=\left\{\overline{\mathbf{E}}: Q \varphi_{0}^{\operatorname{lin}}(\overline{\mathbf{E}})=0\right\} .
$$

This is exactly the set of strains that the material can accommodate by making a mixture of martensitic variants. In general, this set $\mathcal{S}_{f}$ is hard to determine. However, if all variants $\overline{\mathbf{E}}^{(i)}$ are pair-wise compatible, i.e.,

$$
\overline{\mathbf{E}}^{(i)}-\overline{\mathbf{E}}^{(j)}=\mathbf{a} \otimes \mathbf{n}+\mathbf{n} \otimes \mathbf{a}
$$

for some vectors $\mathbf{a}$ and $\mathbf{n}$ and for all $i, j=1, \cdots, k$, then the associated $\mathcal{S}_{f}$ is simply their convex hull [8]. One can show that this is true for materials undergoing cubictetragonal, cubic-trigonal and cubic-orthorhombic transformation irrespective of the orientation of the film. Unfortunately, cubic-to-monoclinic martensites which are the most commonly used shape-memory materials have transformation strains that are not pair-wise compatible in general. But they can be compatible in certain orientations in thin films. Indeed, let $\mathbf{R}$ denote the crystal orientation, the orientation that takes the film basis $\left\{\mathbf{e}_{1}, \mathbf{e}_{2}, \mathbf{e}_{3}\right\}$ to the crystal basis $\left\{\mathbf{f}_{1}, \mathbf{f}_{2}, \mathbf{f}_{3}\right\}, \mathbf{f}_{i}=\mathbf{R e}_{i}$ for $i=1,2,3$, and notice that

$$
\frac{1}{2}\left|\mathbf{R}^{T} \mathbf{E R}-\mathbf{E}^{(i)}\right|^{2}=\frac{1}{2}\left|\mathbf{E}-\mathbf{R} \mathbf{E}^{(i)} \mathbf{R}^{T}\right|^{2}=\frac{1}{2}\left|\mathbf{E}-\mathbf{U}^{(i)}\right|^{2},
$$

where $\mathbf{U}^{(i)}=\mathbf{R} \mathbf{E}^{(i)} \mathbf{R}^{T}$. It is well known that two $2 \times 2$ symmetric matrices $\overline{\mathbf{U}}$ and $\overline{\mathbf{V}}$ are compatible if and only if $\operatorname{det}(\overline{\mathbf{U}}-\overline{\mathbf{V}}) \leqq 0$ [29]. Setting $\mathbf{f}_{i}^{*}=\mathbf{R}^{T} \mathbf{e}_{i}$ for $i=1,2,3$ and $\mathbf{A}=\left(\mathbf{E}^{(i)}-\mathbf{E}^{(j)}\right)$, we have

$$
\begin{aligned}
\operatorname{det}\left(\Pi^{T} \mathbf{R A R} \mathbf{R}^{T} \Pi\right) \leqq 0 & \Longleftrightarrow \operatorname{det}\left(\begin{array}{l}
\mathbf{f}_{1}^{*} \cdot \mathbf{A} \mathbf{f}_{1}^{*} \mathbf{f}_{1}^{*} \cdot \mathbf{A} \mathbf{f}_{2}^{*} \\
\mathbf{f}_{2}^{*} \cdot \mathbf{A} \mathbf{f}_{1}^{*} \mathbf{f}_{2}^{*} \cdot \mathbf{A} \mathbf{f}_{2}^{*}
\end{array}\right) \leqq 0 \\
& \Longleftrightarrow \mathbf{f}_{3}^{*} \cdot \operatorname{adj}\left(\mathbf{E}^{(i)}-\mathbf{E}^{(j)}\right) \mathbf{f}_{3}^{*} \leqq 0 .
\end{aligned}
$$

Notice that (7.28) is the linearized version of (5.12) in Proposition 5.1 of BHATTACHARYA \& JAMES [9]. It follows that $\mathcal{S}_{f}$ is the convex hull of transformation 
strains $\Pi^{T} \mathbf{U}^{(i)} \Pi$ if (7.28) is satisfied for all $i, j=1, \cdots, k$. The vector $\mathbf{f}_{3}^{*}$ is interpreted as follows. Let $\{\mathrm{hkl}\}$ denote the film normal in the cubic crystal basis, i.e.,

$$
\mathbf{e}_{3} \| h \mathbf{f}_{1}+k \mathbf{f}_{2}+l \mathbf{f}_{3} .
$$

Using the definition of $\mathbf{f}_{i}$ and $\mathbf{f}_{i}^{*}$ yields

$$
\mathbf{f}_{3}^{*} \| h \mathbf{e}_{1}+k \mathbf{e}_{2}+l \mathbf{e}_{3} .
$$

It turns out that all pairs of monoclinic variants are pair-wise compatible for $\{100\}$ and $\{110\}$ films in Ti-Ni and $\{110\}$ and $\{111\}$ films in $\mathrm{Cu}-\mathrm{Zn}-\mathrm{Al}$ [38] and $\mathcal{S}_{f}$ is the convex hull of these variants. In contrast, not all pairs are compatible for $\{111\}$ films in Ti-Ni and $\{100\}$ films in $\mathrm{Cu}-\mathrm{Zn}-\mathrm{Al}$. In these cases, the set $\mathcal{S}_{f}$ becomes more complicated [38].

We now turn to polycrystalline thin films. We define the set of recoverable strains of a polycrystalline thin film

$$
\mathcal{P}_{f}=\{\overline{\mathbf{E}}: \bar{\varphi}(\overline{\mathbf{E}})=0\} .
$$

Our task is to determine the set $\mathcal{P}_{f}$. However, this is very difficult and its calculation requires knowledge of the shape and orientation of each grain which is also difficult to obtain. One possibility is to estimate the set $\mathcal{P}_{f}$ using texture-independent bounds. The bound which we believe to be the most useful bound is the Taylor bound based on the use of a constant-strain test field [11]. This gives an upper bound for $\bar{\varphi}$ but is an inner bound for $\mathcal{P}_{f}$. The accuracy of the Taylor bound can be assessed in the recent work by BHATTACHARYA \& KOHN [11] for geometrically linear theory and by KoHn \& NieThammer [30] for geometrically nonlinear theory. Indeed, in our model example above, one can show that the exact recoverable strain is the same as that predicted using the Taylor bound for $\beta=0$ and $\infty$. Unfortunately, the Taylor bound is only suitable for homogenization; and consequently, there is no analogy for the case $0<\beta<\infty$.

We now give the expression of the Taylor bound on two extreme cases, $\beta=0$ and $\beta=\infty$, assuming $\alpha=\frac{\kappa}{d} \stackrel{h}{\rightarrow} 0$ and all grains are columnar in both cases. Consider $\beta=0$ first. The Taylor bound on the set $\mathcal{P}_{f}$ is

$$
\mathcal{T}^{0}=\bigcap_{\mathbf{z}_{p} \in Z} \mathcal{S}_{f}\left(\mathbf{z}_{p}\right)=\left\{\overline{\mathbf{E}}: Q \varphi_{0}^{\operatorname{lin}}\left(\overline{\mathbf{E}} ; \mathbf{R}\left(\mathbf{z}_{p}\right)\right)=0, \text { for a.e. } \mathbf{z}_{p} \in Z\right\}
$$

where

$$
\varphi_{0}^{\operatorname{lin}}\left(\overline{\mathbf{E}} ; \mathbf{R}\left(\mathbf{z}_{p}\right)\right)=\min _{i=1, \cdots, k} \frac{1}{2}\left|\overline{\mathbf{E}}-\Pi^{T}\left(\mathbf{R}\left(\mathbf{z}_{p}\right) \mathbf{E}^{(i)} \mathbf{R}^{T}\left(\mathbf{z}_{p}\right)\right) \Pi\right|^{2} .
$$

In sputtered films, very often all grains have a common crystallographic axis $\{\mathrm{hkl}\}$ as the film normal. Therefore, the set $\mathcal{S}_{f}\left(\mathbf{z}_{p}\right)$ can be determined exactly for all $\mathbf{z}_{p}$ in (7.30) for certain textured cubic-monoclinic films. Consequently, $\mathcal{T}^{0}$ is fully determined. 
Now consider $\beta=\infty$. Let $\mathcal{P}$ be the set of recoverable strains in bulk or thick films: $\mathcal{P}=\left\{\mathbf{E}: \varphi^{H}(\mathbf{E})=0\right\}$ where $\varphi^{H}$ is given by (6.3) ${ }_{3}$ in terms of geometrically linear theory. Then, it can be shown easily that

$$
\Pi^{T} \mathcal{P} \Pi=\left\{\overline{\mathbf{E}}: \varphi_{0}^{H}(\overline{\mathbf{E}})=0\right\} .
$$

So if this function $\varphi_{0}^{H}$ is convex, then the implication is that $\mathcal{P}_{f}=\left\{\overline{\mathbf{E}}: Q \varphi_{0}^{H}(\overline{\mathbf{E}})=\right.$ $0\}=\Pi^{T} \mathcal{P} \Pi$. But in general $\mathcal{P}_{f} \supset \Pi^{T} \mathcal{P} \Pi \supset \Pi^{T} \mathcal{T}^{\infty} \Pi$ where $\mathcal{T}^{\infty}$ is the Taylor bound on $\mathcal{P}$ and is given by

$$
\mathcal{T}^{\infty}=\bigcap_{\mathbf{z}_{p} \in Z} \mathcal{S}\left(\mathbf{z}_{p}\right)=\left\{\mathbf{E}: Q \varphi^{\operatorname{lin}}\left(\mathbf{R}\left(\mathbf{z}_{p}\right)^{T} \mathbf{E R}\left(\mathbf{z}_{p}\right)\right)=0 \text { for a.e. } \mathbf{z}_{p} \in Z\right\}
$$

When subjected to uniaxial in-plane tension in the $\xi$ direction, the Taylor bound of maximum recoverable extension is

$$
\begin{aligned}
e_{R}^{0}=\max _{\overline{\mathbf{E}} \in \mathcal{T}^{0}}(\xi \cdot \overline{\mathbf{E}} \xi) & \text { for } \beta=0, \\
e_{R}^{\infty}=\max _{\overline{\mathbf{E}} \in \Pi^{T} \mathcal{T}^{\infty} \Pi}(\xi \cdot \overline{\mathbf{E}} \xi) & \text { for } \beta=\infty .
\end{aligned}
$$

Table 2 contrasts the behavior of films with long or rod-like $(h>>d)$ grains and films with flat or pancake shaped $(h<<d)$ grains [37]. It lists the predicted recoverable strains for films with different textures in Ti-Ni and $\mathrm{Cu}-\mathrm{Zn}-\mathrm{Al}$. Note that they are larger for flat grains compared to long grains as we expected. We also note here that neither the random nor $\{110\}$ sputtered texture which is common for BCC materials $[27,39]$ are ideal textures for large recoverable strain. The ideal textures appear to be $\{100\}$ for $\mathrm{Cu}-\mathrm{Zn}-\mathrm{Al}$ (this texture can be produced by melt-spinning) and $\{111\}$ for Ti-Ni.

Before closing this subsection, we should mention the effect of the ratio $\frac{\kappa}{d}$ of the size of the microstructure to that of the grain. Above, we took this ratio to be zero; however, this may not be appropriate when the grain size becomes very

Table 2. The predicted uniaxial recoverable extension for various textures. $\varepsilon_{R}^{\infty}$ and $\varepsilon_{R}^{0}$ are the Taylor bounds for films with $\beta=\infty$ and $\beta=0$ respectively. Notice that $\mathcal{S}_{\text {mono }}$ is unknown in bulk cubic-monoclinic martensites. So we use the bigger set $\mathcal{S}_{\text {mono }}^{c}$ which is the convex hull of all monoclinic variants in (7.32) and (7.33) 2 to calculate $\varepsilon_{R}^{\infty}$ (see [37] for more details).

\begin{tabular}{c|cc|cc}
\hline \multirow{2}{*}{ Texture } & \multicolumn{3}{|c}{ Recoverable strains (\%) } \\
\cline { 2 - 5 } & \multicolumn{2}{|c|}{ Ti-Ni } & \multicolumn{2}{c}{ Cu-Zn-Al } \\
\hline & $\varepsilon_{R}^{\infty}$ & $\varepsilon_{R}^{0}$ & $\varepsilon_{R}^{\infty}$ & $\varepsilon_{R}^{0}$ \\
\hline random & 2.3 & 2.3 & 1.3 & 1.7 \\
\hline$\{111\}$ film & 5.3 & 8.1 & 1.9 & 5.9 \\
\hline$\{100\}$ film & 2.3 & 2.3 & 7.1 & 7.1 \\
\hline$\{110\}$ sputtered film & 2.3 & 2.3 & 1.7 & 1.7 \\
\hline
\end{tabular}


small (on the order of tens of nanometer). In that situation $(\kappa>>d)$, Theorem 1 shows that it costs materials more energy to form microstructure inside each grain to accommodate deformation and consequently strains cannot be recovered unless the texture is exceptional.

\subsection{Effective Conductivity}

We now turn to study the influence of different ratios of length scales on the conductivity of heterogeneous thin films. It will be evident that similar results should also hold in other linear properties.

Consider a conducting thin strip made by mixing two anisotropic conducting materials as shown in Fig. 4(a). Let $P_{i j}$ and $Q_{i j}$ be the anisotropic conductivities in the "grey" and "white" regions of Fig. 4(a). Let $0 \leqq \lambda \leqq 1$ be the volume fraction of the grey region. The energy is given by

$$
\varphi\left(\mathbf{f}, z_{1}\right)= \begin{cases}\frac{1}{2}\left(P_{11} f_{1}^{2}+2 P_{12} f_{1} f_{2}+P_{22} f_{2}^{2}\right), & m \leqq z_{1}<m+\lambda, \\ \frac{1}{2}\left(Q_{11} f_{1}^{2}+2 Q_{12} f_{1} f_{2}+Q_{22} f_{2}^{2}\right), & m+\lambda \leqq z_{1}<m+1,\end{cases}
$$

where $m \in \mathbb{Z}$ and $\mathbf{f}$ is interpreted as the electric field. The usual symmetry assumption $P_{i j}=P_{j i}$ and $Q_{i j}=Q_{j i}$ is assumed and the positive definiteness of the energy density requires

$$
\Delta_{P}=P_{11} P_{22}-P_{12}^{2}>0, \quad P_{11}>0, \quad \Delta_{Q}=Q_{11} Q_{22}-Q_{12}^{2}>0, \quad Q_{11}>0 .
$$

For the conducting strip shown in Fig. 4(a), Theorem 3 implies that the effective energy density takes the form: $\bar{\varphi}(\xi)=\frac{1}{2} \bar{C}^{\beta} \xi^{2}$ where $\bar{C}^{\beta}$ is the effective conductivity at the ratio $\beta$. The following proposition shows that $\bar{C}^{\beta}$ depends on $\beta$.

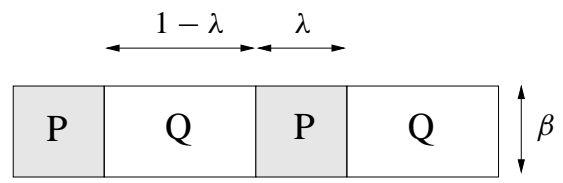

(a)

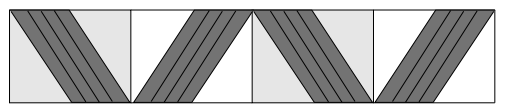

$\eta$

(b)

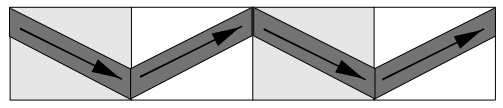

(c)

Fig. 4. (a) A heterogeneous thin film mixing with two anisotropic conducting materials. (b) The test field $\nabla \eta$. The darkly shaded regions form the support of $\nabla \eta$ and the straight lines within them are level sets of $\eta$. (c) The test divergence-free flow $\mathbf{g}$. The darkly shaded strips form the support of $\mathbf{g}$ and the arrows within them point out the direction of the flow. 
Proposition 2. For the thin strip shown in Fig. 4(a), the effective conductivity $\bar{C}^{\beta}$ satisfies

$$
\bar{C}^{0} \leqq \bar{C}^{\beta} \leqq \bar{C}^{\infty} \text { for } 0<\beta<\infty
$$

where

$$
\begin{aligned}
\bar{C}^{\infty} & =\frac{C_{11}^{\infty} C_{22}^{\infty}-C_{12}^{\infty^{2}}}{C_{22}^{\infty}} \\
\bar{C}^{0} & =\frac{\bar{P} \bar{Q}}{\lambda \bar{Q}+(1-\lambda) \bar{P}}
\end{aligned}
$$

and $\mathbf{C}^{\infty}$ is defined by

$$
\mathbf{C}^{\infty}=(\lambda \mathbf{P}+(1-\lambda) \mathbf{Q} \cdot \mathbf{S}) \cdot(\lambda \mathbf{I}+(1-\lambda) \mathbf{S})^{-1}
$$

with $\mathbf{S}$ given by

$$
\mathbf{S}=\left(\begin{array}{cc}
\frac{P_{11}}{Q_{11}} & \frac{P_{12}-Q_{12}}{Q_{11}} \\
0 & 1
\end{array}\right)
$$

and $\bar{P}$ and $\bar{Q}$ defined by

$$
\bar{P}=\frac{P_{11} P_{22}-P_{12}^{2}}{P_{22}}=\frac{\Delta_{P}}{P_{22}} \quad \text { and } \bar{Q}=\frac{Q_{11} Q_{22}-Q_{12}^{2}}{Q_{22}}=\frac{\Delta_{Q}}{Q_{22}} .
$$

Further, if $Q_{12} \Delta_{P}-P_{12} \Delta_{Q}=0$, then

$$
\bar{C}^{0}=\bar{C}^{\beta}=\bar{C}^{\infty} \text {. }
$$

Finally, if the volume fraction $\lambda=\frac{1}{2}$, then

$$
\begin{aligned}
& \bar{C}^{0} \leqq \bar{C}^{\beta} \leqq \bar{C}^{0}\left(1-\beta \bar{C}^{0}\left(\frac{\frac{\left|P_{12}\right|}{P_{22}}}{\bar{P}}+\frac{\frac{\left|Q_{12}\right|}{Q_{22}}}{\bar{Q}}\right)\right)^{-1} \quad \text { for } \beta \text { small }, \\
& \bar{C}^{\infty} \geqq \bar{C}^{\beta} \geqq \bar{C}^{\infty}\left(1-\frac{\left|Q_{12} \Delta_{P}-P_{12} \Delta_{Q}\right|}{2 \beta\left(P_{11} \Delta_{Q}+Q_{11} \Delta_{P}\right)}\right) \quad \text { for } \beta \text { large } .
\end{aligned}
$$

Proof. Let us calculate $\bar{C}^{\infty}$ first. When $\beta=\frac{h}{d} \stackrel{h}{\rightarrow} \infty$, the macroscopic energy density is obtained by homogenizing the bulk conductor and then by passing to the thin-film limit (see Section 6). Let $\mathbf{C}^{\infty}$ be the effective conductivity of the bulk material. We can calculate it by noticing that we have a rank-one laminate in two dimensions. Assuming constant gradient in each layer [34], we can obtain $\mathbf{C}^{\infty}$ defined by (7.39). The bulk energy density $\varphi^{H}$ becomes

$$
\varphi^{H}(\mathbf{f})=\frac{1}{2}\left\{C_{11}^{\infty} f_{1}^{2}+2 C_{12}^{\infty} f_{1} f_{2}+C_{22}^{\infty} f_{2}^{2}\right\} .
$$


The effective energy density for this thin conducting strip is obtained by passing to the thin-film limit, i.e.,

$$
\bar{\varphi}^{\infty}(\xi)=Q \varphi_{0}^{H}(\xi)=\varphi_{0}^{H}(\xi)=\inf _{b \in \mathbb{R}} \frac{1}{2}\left\{C_{11}^{\infty} \xi^{2}+2 C_{12}^{\infty} \xi b+C_{22}^{\infty} b^{2}\right\}=\frac{1}{2} \bar{C}^{\infty} \xi^{2},
$$

where the effective conductivity $\bar{C}^{\infty}$ is defined by (7.37).

On the other hand, if the ratio $\beta=\frac{h}{d} \rightarrow 0$ as $h \rightarrow 0$, the effective energy density is obtained by passing to the thin-film limit first, followed by homogenization (see Section 4). Indeed, after passing to the thin-film limit, the local energy densities within the grey and white regions become

$$
\frac{1}{2} \bar{P} \xi^{2} \quad \text { and } \quad \frac{1}{2} \bar{Q} \xi^{2},
$$

where $\bar{P}$ and $\bar{Q}$ are defined by (7.40). Now the effective energy density is obtained by homogenization and is given by

$$
\bar{\varphi}^{0}(\xi)=\frac{1}{2} \bar{C}^{0} \xi^{2},
$$

where the effective conductivity $\bar{C}^{0}$ is defined by (7.38).

We now show (7.36). The Fenchel transformation of $\varphi$ is given by

$$
\varphi^{*}\left(\mathbf{g}, z_{1}\right)= \begin{cases}\frac{1}{2 \Delta_{P}}\left(P_{22} g_{1}^{2}-2 P_{12} g_{1} g_{2}+P_{11} g_{2}^{2}\right), & m \leqq z_{1}<m+\lambda, \\ \frac{1}{2 \Delta_{Q}}\left(Q_{22} g_{1}^{2}-2 Q_{12} g_{1} g_{2}+Q_{11} g_{2}^{2}\right), & m+\lambda \leqq z_{1}<m+1 .\end{cases}
$$

Substituting $\mathbf{g}=(\tau, 0)^{T}$ into the lower bound (7.7) and optimizing over $\tau$ yields $\bar{C}^{\beta} \geqq \bar{C}^{0}$ for all $\beta>0$. Further, consider the test function $\mathbf{f}_{P}=\left(a_{P}, b\right)^{T}$ and $\mathbf{f}_{Q}=\left(a_{Q}, b\right)^{T}$. Using the definition of $\bar{\varphi}$ in Theorem 3 and minimizing the energy over all possible $a_{P}, a_{Q}$ and $b$, we have $\bar{C}^{\beta} \leqq \bar{C}^{\infty}$ for all $\beta>0$.

Suppose $Q_{12} \Delta_{P}=P_{12} \Delta_{Q}$. Direct calculation on $\bar{C}^{0}$ and $\bar{C}^{\infty}$ shows that these are equal, and this implies (7.41) due to (7.36). An interesting example of this type is $P_{12}=Q_{12}=0$ when there is no coupling between the thickness and the in-plane directions; so the effective conductivity does not depend on the ratio $\beta$.

We prove bounds (7.42) and (7.43) for $\lambda=\frac{1}{2}$. Consider (7.42) first. To show the right-hand side of (7.42), consider the test function $\eta$ constructed by

$$
\nabla \eta=\left\{\begin{array}{cl}
\frac{\tau}{\bar{P}}\left(1,-\frac{P_{12}}{P_{22}}\right)^{T} & \text { in the darkly shaded region of the grey domain } \\
\frac{\tau}{\bar{Q}}\left(1,-\frac{Q_{12}}{Q_{22}}\right)^{T} & \text { in the darkly shaded region of the white domain } \\
0 & \text { otherwise }
\end{array}\right.
$$

as shown in Fig. 4(b). The area fraction of these darkly shaded regions is $A_{1}=$ $\frac{1}{2}-\beta \frac{\left|P_{12}\right|}{P_{22}}$ and $A_{2}=\frac{1}{2}-\beta \frac{\left|Q_{12}\right|}{Q_{22}}$ respectively. Using this test function, we have

$$
\bar{\varphi}\left(f_{1}\right)=\frac{1}{2} \bar{C}^{\beta} f_{1}^{2} \leqq \frac{1}{2}\left(\frac{A_{1}}{\bar{P}}+\frac{A_{2}}{\bar{Q}}\right) \tau^{2},
$$


where

$$
f_{1}=\left(\frac{A_{1}}{\bar{P}}+\frac{A_{2}}{\bar{Q}}\right) \tau .
$$

The inequality (7.42) is obtained by substituting (7.47) into (7.46) and using the definition of $\bar{C}^{0}$ in (7.38) for $\lambda=\frac{1}{2}$.

We now turn to (7.43). To show the right-hand side of (7.43), consider the lower bound (7.7) and the Fenchel transformation of $\varphi$ given by (7.44). The test divergence-free flow $\mathbf{g}$ is chosen to be

$$
\mathbf{g}=\left\{\begin{array}{cl}
(\sigma,-\varepsilon)^{T} & \text { in the darkly shaded region of the grey domain, } \\
(\sigma, \varepsilon)^{T} & \text { in the darkly shaded region of the white domain, } \\
0 & \text { otherwise, }
\end{array}\right.
$$

as shown in Fig. 4(c). Assume $Q_{12} \Delta_{P}-P_{12} \Delta_{Q} \geqq 0$ (otherwise change $\varepsilon$ to $-\varepsilon$ in (7.48) and the corresponding flow direction in Fig. 4(c)); choose

$$
\varepsilon=\frac{Q_{12} \Delta_{P}-P_{12} \Delta_{Q}}{\left(P_{11} \Delta_{Q}+Q_{11} \Delta_{P}\right)} \sigma
$$

Then, the area fraction of the support of $\mathbf{g}$ is $\left(1-\frac{\varepsilon}{2 \beta \sigma}\right)$. Substituting (7.48) and (7.49) into (7.7) gives

$$
\bar{\varphi}(\xi)=\frac{1}{2} \bar{C}^{\beta} \xi^{2} \geqq \frac{1}{2}\left(1-\frac{\varepsilon}{2 \beta \sigma}\right)\left(2 \xi \sigma-\frac{\sigma^{2}}{\bar{C}^{\infty}}\right) .
$$

Maximizing the above inequality over $\sigma$ gives (7.43).

We comment on the result of Damlamian \& Vogelius 0[20] who obtained optimal bounds for a composite thin structure made of two isotropic conductors. They consider the case $\varphi=\varphi\left(\mathbf{f}, \frac{\mathbf{z}_{p}}{h}, z_{3}\right)(\beta=1$ in our notation and fully three dimensions). However, their optimal bounds are $\beta$-independent and hence their bounds hold for all $\beta$. Further, their optimal microstructure are $z_{3}$-independent. Our Proposition 2 above shows that their $\beta$-independent bound may not be optimal if the conductivity is anisotropic and the film normal is not an eigenvector of the conductivity tensor. We speculate that their lower bound is optimal only for $\beta=0$ and upper bound only for $\beta=\infty$. The problem of deriving good $\beta$-dependent bounds are complicated due to reasons discussed at the end of Section 7.1.1.

Finally, it is clear that similar results must hold for elasticity, and there is some suggestive experimental evidence in this direction.

\subsection{Multilayers}

Consider a multilayered thin film made up of a finite number of alternating layers of a martensitic material and a purely elastic material. Let $\lambda$ be the volume fraction of the martensitic material and let $\varepsilon_{m}$ be the mismatch strain of the elastic material relative to the austenite phase of the martensitic material. The effective behavior is some combination of the behavior of these two materials; however, the 

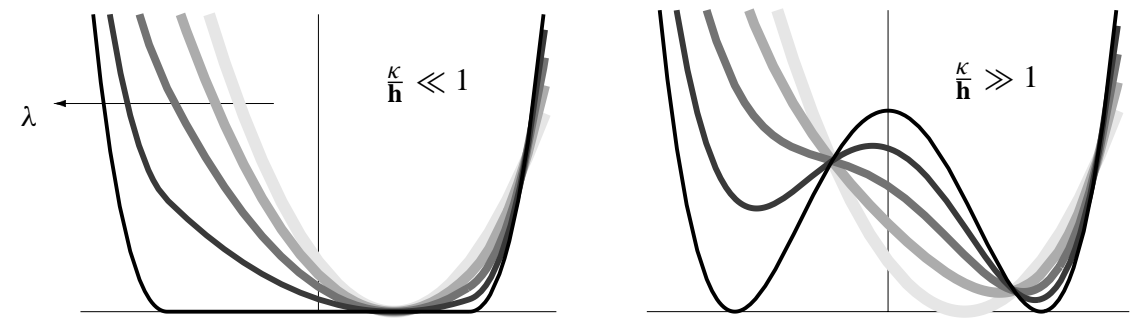

Fig. 5. The effective behavior of a multilayer thin film is determined by the energies above for small and large values of $\frac{\kappa}{h}$.

nature of the behavior depends on the ratio $\alpha^{\prime}=\frac{\kappa}{h}$ of the microstructure size to the thickness. For example, assume the local energy density $\varphi: \mathbb{R}^{2} \times(0,1) \rightarrow \mathbb{R}$ has the following form

$$
\varphi\left(\mathbf{f}, z_{3}\right)=\left\{\begin{array}{l}
\varphi_{\text {mart }}(\mathbf{f})=\frac{1}{2}\left(\left(f_{1}^{2}-1\right)^{2}+f_{2}^{2}\right), \text { for } \frac{m-1}{M} \leqq z_{3}<\frac{(m-1)+\lambda}{M} \\
\varphi_{\text {elas }}(\mathbf{f})=\frac{1}{2}\left(\left(f_{1}-\varepsilon_{m}\right)^{2}+f_{2}^{2}\right), \text { for } \frac{(m-1)+\lambda}{M} \leqq z_{3}<\frac{m}{M}
\end{array}\right.
$$

for $m=1, \cdots, M$. From Theorem 4, the effective energy densities for small and large values of $\alpha^{\prime}$ are given by

$$
\begin{aligned}
& \bar{\varphi}^{0}(\xi)=\frac{\lambda}{2}\left\{\left(\xi^{2}-1\right)_{+}\right\}^{2}+\frac{1-\lambda}{2}\left(\xi-\varepsilon_{m}\right)^{2} \quad \text { for } \alpha^{\prime}=0, \\
& \bar{\varphi}^{\infty}(\xi)=Q\left\{\frac{\lambda}{2}\left(\xi^{2}-1\right)^{2}+\frac{1-\lambda}{2}\left(\xi-\varepsilon_{m}\right)^{2}\right\} \quad \text { for } \alpha^{\prime}=\infty \text {, }
\end{aligned}
$$

where $Q$ denotes the operation of quasi-convexification. The result is also schematically shown in Fig. 5. The effective energy for $\alpha^{\prime}$ small is shown on the left while that for $\alpha^{\prime}$ large is obtained by the quasi-convexification (or convexification in this case) of the multi-well energy shown on the right-hand side of Fig. 5. The thin dark line is the energy of the martensitic material and the thick light line is the energy of the elastic material. The behavior of the multilayer is shown by lines of increasing greylevel for decreasing volume fraction $\lambda$ (also see [9]). For small $\alpha^{\prime}$, the martensitic material freely forms microstructure and the multilayer can accommodate this mismatch strain $\varepsilon_{m}$ and behaves like an elastic material with soft-modulus. For large $\alpha^{\prime}$ on the other hand, the multilayer behaves like a phase transforming material: it has two variants with transformation strains which may be different from that of the original martensitic material, and one variant is preferred over the other. Hence, this multilayer film will display a two-way shape-memory effect. Further notice that the multilayer is internally stressed so that the minimum energy is not zero. Finally, the multilayer can form "macroscopic twins": these are not twins confined to the martensitic material but encompass both the elastic and the martensitic material. Thus, multilayers promise to be a means of making apparently new materials with interesting and novel properties. 
Acknowledgements. Part of this work was carried out under the guidance of K. BhATTACHARYA while I stayed at the California Institute of Technology in Pasadena and MaxPlanck-Institute for Mathematics in the Sciences in Leipzig. I wish to thank him for his invaluable advice and constant encouragement. I would also like to thank S. MülLER for sharing with me some of his insights and A. BRAIDES for showing me the proof of Theorem 5. Finally, I am grateful to I. FonseCA, G. FRANCFORT and R. V. KoHN for helpful discussions. I am glad to acknowledge the partial support of the Powell Foundation and the National Science Foundation through CMS-9457573.

\section{References}

1. E. Acerbi, G. Buttazzo \& Percivale. A Variational Definition of the Strain Energy for an Elastic String. J. Elasticity 25, 137-148, 1991.

2. E. ACERbi \& N. FusCo. Semicontinuity Problems in the Calculus of Variations. Arch. Rat. Mech. Anal. 86, 125-145, 1984.

3. G. ARLt. Twinning in the Ferroelectric and Ferroelastic Ceramics: Stress Relief. $J$. Mater. Sci. 25, 2655-2666, 1990.

4. H. Aттоuch. Variational Convergence for Functions and Operators. Pitman, London, 1984.

5. J. M. Ball \& R. D. James. Fine Phase Mixtures as Minimizers of Energy. Arch. Rat. Mech. Anal. 100, 13-5, 1987.

6. J. M. Ball \& R. D. James, Proposed Experimental Tests of a Theory of Fine Microstructure and the Two Well Problem. Phil. Trans. Royal Soc. London A. 338, 389-450, 1992.

7. J. M. Ball \& F. Murat. $W^{1, p}$-quasiconvexity and Variational Problems for Multiple Integrals. J. Funct. Anal. 58, 225-253, 1984.

8. K. Bhattacharya. Comparison of the Geometrically Nonlinear and Linear Theories of Martensitic Transformation. Cont. Mech. Thermodyn. 5, 205-242, 1993.

9. K. Bhattacharya \& R. D. James. A Theory of Thin Films of Martensitic Materials with Applications to Microactuators. J. Mech. Phys. Solids 47, 531-576, 1999.

10. K. Bhattacharya \& R. V. Kohn. Symmetry, Texture and the Recoverable Strain of Shape-Memory Polycrystals. Acta Mater. 44, 529-542, 1996.

11. K. Bhattacharya \& R. V. Kohn. Elastic Energy Minimization and the Recoverable Strains of Polycrystalline Shape-Memory Materials. Arch. Rational Mech. Anal. 139, 99-180, 1997.

12. A. BRAIDES. Private Communciation. 1998.

13. A. Braides, Homogenization of Some Almost Periodic Coercive Functional. Rend. Accad. Naz. Sci. XL 103, 313-322 1985.

14. A. Braides \& A. Defranceschi. Homogenization of Multiple Integrals. Oxford University Press, Oxford, 1998.

15. A. Braides, I. Fonseca \& G. FrancFort. 3D-2D Asymptotic Analysis for Inhomogeneous Thin Films. Submitted for publication, 1999.

16. D. CAillerie. Thin Elastic and Periodic Plates. Math. Meth. in the Appl. Sci. 6, 159-191, 1994

17. L. W. Chang \& D. S. Grummon. Phase-Transformations in Sputtered Thin-Films of Ti-X(Ni,Cu)(1-X) .2. Displacive Transformations, Phil. Mag. A 76, 191-219, 1997.

18. L. W. Chang \& D. S. Grummon. Structure Evolution in Sputtered Thin-Films of Ti$\mathrm{X}(\mathrm{Ni}, \mathrm{Cu})(1-\mathrm{X})$.1. Diffusive Transformations. Phil. Mag. A 76, 163-189, 1997.

19. B. DaCorogna. Quasiconvexity and Relaxation of Nonconvex Problems in the Calculus of Variations. J. Funct. Anal. 46, 102-118, 1982.

20. A. Damlamian \& M. Vogelius. Homogenization Limits of Diffusion Equations in Thin Domains. Mathematical Modeling and Numerical Analysis 22, 53-74, 1988.

21. H. Le Dret \& A. Raoult. The Nonlinear Membrane Model as Variational Limit of Nonlinear Three-dimensional Elasticity. J. Math. Pures. Appl. 74, 549-578, 1995. 
22. I. Fonseca \& G. Francfort. 3D-2D Asymptotic Analysis of an Optimal Design Problem for Thin Films. J. Reine Angew. Math. 505, 173-202, 1998.

23. I. Fonseca, S. Müller \& P. Pedregal. Analysis of Concentration and Oscillation Effects Generated by Gradients. Submitted to SIAM J. Math. Pures Appl. 29, 736-756, 1998.

24. G. A. Francfort \& S. MÜller. Combined Effects of Homogenization and Singular Perturbations in Elasticity. J. Reine Angew. Math. 454, 1-35, 1994.

25. E. De Giongi. Sulla convergenza di alcune successioni di integrali del tipo dell'area. Rendi Conti di Mat. 8, 277-294, 1975.

26. E. De Giorgi \& T. Franzoni. Su un tipo di convergenza variazionale. Atti. Accad. Naz. Lincei Rend. Cl. Sci. Mat. 58, 842-850, 1975.

27. K. R. C. Gisser, J. D. Busch, A. D. Johnson \& A. B. Ellis. Oriented Nickel-Titanium Shape Memory Alloy Films Prepared by Annealing During Deposition. Appl. Phys. Lett. 61, 1632-1634, 1992.

28. A. Ishida, A. TAKei \& S. Miyazaki. Shape Memory Thin Film of Ti-Ni Formed by Sputtering. Thin Solid Films 228, 210-214, 1993.

29. R. V. Korn. The Relaxation of a Double-Well Energy. Cont. Mech. Thermodyn. 3, 981-1000, 1991.

30. R. V. Kohn \& B. Niethammer. Geometrically Nonlinear Shape-Memory Polycrystals made from a Two-variant Material. Submitted for publication, 1999.

31. R. V. Kohn \& M. Vogelius. A new model for Thin Plates with Rapidly Varying Thickness. Int. J. Solids \& Structures 20, 333-350, 1984.

32. R. V. Kohn \& M. Vogelius. A new model for Thin Plates with Rapidly Varying Thickness II: A Convergence Proof. Quarterly of Applied Mathematics 43, 1-22, 1985.

33. P. Krulevitch, A. P. Lee, P. B. Ramsey, J.C. Trevino, J. Hamilton \& M. A. NoRThruP. Thin Film Shape Memory Alloy Microactuators. Journal of Microelectromechanical Systems 5, 270-282, 1996.

34. G. W. Milton. A Link between Sets of Tensors Stable under Lamination and Quasiconvexity. Communications on Pure and Applied Mathematics 47, 959-1003, 1994.

35. S. MüllER. Private Communciation. 1998.

36. S. Müller. Homogenization of Nonconvex Integral Functionals and Cellular Elastic Materials. Arch. Rat. Mech. Anal. 99, 189-212, 1987.

37. Y. C. Shu \& K. Bhattacharya. The Influence of Texture on the Shape-Memory Effect in Polycrystals. Acta Materialia 46, 5457-5473, 1998.

38. Y. C. SHU. Shape-Memory Effect in Bulk and Thin-Film Polycrystals. Ph.D. Thesis. California Institute of Technology, 1998.

39. Q. Su, S., Z. Hua \& M. WutTig. Martensitic Transformation in $\mathrm{Ni}_{50} \mathrm{Ti}_{50}$ Films. $J$. Alloys and Compounds 211/212, 460-463, 1994.

Institute of Applied Mechanics

National Taiwan University

Taipei, Taiwan, ROC

e-mail: yichung@spring.iam.ntu.edu.tw

(Accepted December 8, 1999) 